\title{
Early Dark Energy Does Not Restore Cosmological Concordance
}

\author{
J. Colin Hill, ${ }^{1,2}$ Evan McDonough, ${ }^{3,4}$ Michael W. Toomey, ${ }^{3}$ and Stephon Alexander ${ }^{3}$ \\ ${ }^{1}$ Department of Physics, Columbia University, New York, NY, USA 10027 \\ ${ }^{2}$ Center for Computational Astrophysics, Flatiron Institute, New York, NY, USA 10010 \\ ${ }^{3}$ Brown Theoretical Physics Center and Department of Physics, Brown University, Providence, RI, USA 02912 \\ ${ }^{4}$ Center for Theoretical Physics, Massachusetts Institute of Technology, Cambridge, MA 02139, USA
}

\begin{abstract}
Current cosmological data exhibit a tension between inferences of the Hubble constant, $H_{0}$, derived from early- and late-universe measurements. One proposed solution is to introduce a new component in the early universe, which initially acts as "early dark energy" (EDE), thus decreasing the physical size of the sound horizon imprinted in the cosmic microwave background (CMB) and increasing the inferred $H_{0}$. Previous EDE analyses have shown this model can relax the $H_{0}$ tension, but the CMBpreferred value of the density fluctuation amplitude, $\sigma_{8}$, increases in EDE as compared to $\Lambda$ CDM, increasing tension with large-scale structure (LSS) data. We show that the EDE model fit to CMB and SH0ES data yields scale-dependent changes in the matter power spectrum compared to $\Lambda$ CDM, including $10 \%$ more power at $k=1 \mathrm{~h} / \mathrm{Mpc}$. Motivated by this observation, we reanalyze the EDE scenario, considering LSS data in detail. We also update previous analyses by including Planck 2018 CMB likelihoods, and perform the first search for EDE in Planck data alone, which yields no evidence for EDE. We consider several data set combinations involving the primary CMB, CMB lensing, supernovae, baryon acoustic oscillations, redshift-space distortions, weak lensing, galaxy clustering, and local distance-ladder data (SH0ES). While the EDE component is weakly detected $(3 \sigma)$ when including the SHOES data and excluding most LSS data, this drops below $2 \sigma$ when further LSS data are included. Further, this result is in tension with strong constraints imposed on EDE by CMB and LSS data without SH0ES, which show no evidence for this model. We also show that physical priors on the fundamental scalar field parameters further weaken evidence for EDE. We conclude that the EDE scenario is, at best, no more likely to be concordant with all current cosmological data sets than $\Lambda \mathrm{CDM}$, and appears unlikely to resolve the $H_{0}$ tension.
\end{abstract}

\section{INTRODUCTION}

The value of the Hubble constant $H_{0}$, the present-day expansion rate of the Universe, is crucial to cosmology. All cosmological quantities are connected to $H_{0}$, which effectively sets the scale of the Universe. In recent years, the value of $H_{0}$ inferred from probes of the early universe has been in persistent disagreement with that measured from probes of the late universe, a discrepancy that has now reached $\approx 4-6 \sigma$ significance (e.g., [1]). Assuming that systematic errors in one or more measurements are not responsible for the disagreement, this "Hubble tension" may be a first sign of physics beyond the standard $\Lambda$ Cold Dark Matter $(\Lambda \mathrm{CDM})$ cosmological model.

The cosmic microwave background (CMB) allows for a precise, albeit indirect, inference of the Hubble constant in the context of a cosmological model 227 . The angular size of the sound horizon, combined with constraints on the energy density in each component of the $\Lambda \mathrm{CDM}$ model derived from the CMB temperature, polarization, and lensing power spectra, allow for a determination $H_{0}=67.36 \pm 0.54 \mathrm{~km} / \mathrm{s} / \mathrm{Mpc}$ using the Planck 2018 data alone [5]. The same approach can be taken without CMB anisotropy data, instead using an earlyuniverse measurement of the baryon density, namely, that inferred from Big Bang nucleosynthesis [8], and lateuniverse measurements of the matter density to calibrate the sound horizon measured in baryon acoustic oscillation
(BAO) experiments. ${ }^{1}$ Applied to Dark Energy Survey (DES) data combined with Baryon Oscillation Spectroscopic Survey (BOSS) BAO data, this methodology leads to $H_{0}=67.4_{-1.2}^{+1.1} \mathrm{~km} / \mathrm{s} / \mathrm{Mpc}[10$, in near-perfect agreement with the $\mathrm{CMB}$ constraints, albeit with error bars doubled in size. Recent analyses have further refined this cosmological approach to constrain $H_{0}$ using not only sound horizon information, but also information in the shape of the matter power spectrum, as measured from the redshift-space galaxy power spectrum [11-13]. The results are consistent with those from the Planck CMB analysis, again albeit with somewhat larger error bars.

The opposite approach is to constrain $H_{0}$ directly via late-universe measurements, without assuming a cosmological model. Historically, these constraints have been obtained via the classical distance ladder (e.g., [14]). In this procedure, parallax measurements are used to calibrate the period-luminosity relation of Cepheid variable stars, which are then used to calibrate the luminosity of nearby Type Ia supernovae (SN), allowing distant SNIa to be used as proxies for the Hubble flow. The SH0ES collaboration has applied this method in recent years, most recently obtaining $H_{0}=74.03 \pm 1.42 \mathrm{~km} / \mathrm{s} / \mathrm{Mpc}$ 15. The Cepheid calibration step can alternatively be swapped out for a calibration using the "tip of

\footnotetext{
${ }^{1}$ Note that the early-universe methods also require knowledge of the radiation density, as inferred from the CMB monopole temperature 9].
} 
the red giant branch" in the Hertzsprung-Russell diagram. The most recent analysis with this method yields $H_{0}=69.6 \pm 1.9 \mathrm{~km} / \mathrm{s} / \mathrm{Mpc}$ [16]. Another alternative approach replaces the Cepheids with Miras, variable red giant stars, leading to $H_{0}=73.3 \pm 3.9 \mathrm{~km} / \mathrm{s} / \mathrm{Mpc}$ [17. Very recently, late-universe $H_{0}$ probes have emerged that are independent of, and statistically competitive with, the distance ladder. In particular, the HOLiCOW collaboration has constrained $H_{0}$ by measuring time delays in strongly lensed quasar systems, obtaining $H_{0}=$ $73.3_{-1.8}^{+1.7} \mathrm{~km} / \mathrm{s} / \mathrm{Mpc}$ [18, although recently concerns have been raised regarding the sensitivity to details of the lens modeling [19, 20]. The Megamaser Cosmology Project finds $H_{0}=73.9 \pm 3.0 \mathrm{~km} / \mathrm{s} / \mathrm{Mpc}$ [21] from very-longbaseline interferometry observations of water masers orbiting supermassive black holes. A futuristic, but already fruitful, alternative is to directly infer $H_{0}$ using not the brightness of standard candles, but the "volume" of standard sirens, i.e., gravitational waves from merging binary neutron stars [22, 23]. In this work, we focus on the $H_{0}$ constraint from 15, as this has been the most widely analyzed late-universe measurement to date.

There exist varied theoretical proposals to explain or ameliorate the $H_{0}$ discrepancy, ranging from new physics in the very early to late universe. It has been argued 24 that the proposal "least unlikely to be successful" is an increase in the expansion rate just prior to recombination, which acts to shrink the sound horizon at last scattering. There is now a growing body of work to realize this in concrete theoretical models. A popular subclass of these models has been termed "Early Dark Energy" (EDE) 25], and many EDE-like models have been proposed, both in the context of the $H_{0}$ tension [25-33] and other areas of cosmological phenomenology (e.g., [34 36]).

In the EDE implementation that we will focus on, an ultra-light scalar field, significantly lighter than canonical ultra-light axion or fuzzy dark matter, is displaced from the minimum of its potential at early times, and, held up by Hubble friction, effectively acts as an additional contribution to dark energy. When the Hubble parameter becomes less than the mass of the field, it rolls down its potential and begins to oscillate about the minimum. If the potential about the minimum is steeper than quadratic, the EDE field quickly becomes a subdominant component of the universe; hence the name "early dark energy". The model can be parameterized by the field's maximal fractional contribution to the energy density of the universe, $f_{\mathrm{EDE}} \equiv \max \left(\rho_{\mathrm{EDE}} / 3 M_{p l}^{2} H^{2}\right)$, and the critical redshift $z_{c}$ at which this maximum is reached, which roughly corresponds to the moment before the onset of oscillations. This evolution is encoded in the Hubble parameter as an enhancement (compared to $\Lambda \mathrm{CDM}$ ) localized in redshift-space in the epoch before the onset of oscillations. The consequent decrease in the sound horizon $r_{s}$ increases the inferred $H_{0}$ value from the earlyuniverse approach described above, in principle bringing it into agreement with late-universe measurements.

However, the apparent success of the EDE scenario in resolving the Hubble tension comes at a cost: in order to preserve the fit to CMB data, some of the standard $\Lambda$ CDM parameters shift. In particular, the physical CDM density $\Omega_{c} h^{2}$ increases substantially, as does the spectral index $n_{s}$ and to a lesser extent the physical baryon density $\Omega_{b} h^{2}$ [26. Primarily due to the increase in $\Omega_{c} h^{2}$, the late-time amplitude of density fluctuations, $\sigma_{8}$, increases as well. This increase exacerbates the current mild tension between $\mathrm{CMB}$ and large-scale structure (LSS) inferences of this parameter. Thus, one expects that the fit to LSS data will be degraded in EDE models that fit the CMB and the distance-ladder $H_{0}$ data. We confirm this expectation in this paper.

The physical origin of these parameter shifts is fairly straightforward, and likely applies to any scenario in which the sound horizon is decreased through the introduction of a new dark-energy-like component in the pre-recombination universe (so as to increase the expansion rate during this epoch over that in $\Lambda \mathrm{CDM}$ ). This new component (e.g., EDE) acts to slightly suppress the growth of perturbations during the period in which it contributes non-negligibly to the cosmic energy density. Thus, in order to preserve the fit to the CMB data, the CDM density must be increased to compensate for this loss in the efficiency of perturbation growth. Since the EDE field is only relevant for a short period of time, the suppression is scale-dependent, and thus a small change in $n_{s}$ is also required to preserve the CMB fit. While we carefully quantify these effects in the EDE scenario here, the basic physics indicates that similar considerations would afflict any $H_{0}$-tension-resolving scenario that works in a similar manner.

A simple way to quantify the parameter shifts in the EDE scenario and the associated CMB-LSS tension is by examining $S_{8} \equiv \sigma_{8}\left(\Omega_{m} / 0.3\right)^{0.5}$. Fitting the EDE model (with index $n=3$, see Eq. (5) below) to CMB and cosmological distance data, 26 finds $S_{8}=0.842 \pm 0.014$, which is in $2.3 \sigma$ tension with the DES-Y1 constraint $S_{8}=0.773_{-0.020}^{+0.026} 38$. Moreover, $S_{8}$ is only a single number, while LSS data constrain the matter power spectrum $P(k)$ across a decade in $k$-space. As we show, EDE models that fit the CMB and SHOES data produce significant effects on $P(k)$ beyond an overall amplitude change (as $S_{8}$ primarily captures), thereby suggesting the possibility of tightly constraining these models using LSS data. We note that late-universe constraints on early-universe resolutions have also been discussed in, e.g., [39, 40.

In this work, by "large-scale structure data" we refer to data sets that constrain not only the expansion history of the universe, e.g., via the BAO feature, but also the growth history, e.g., via weak gravitational lensing (including CMB lensing), photometric and/or spectroscopic galaxy clustering, galaxy cluster counts, etc. In recent years, LSS data sets have delivered precise cosmological constraints, and any extension of the standard cosmological model must also satisfy these bounds. Recent LSS breakthroughs have come from BOSS 41, a component of the Sloan Digital Sky Survey (SDSS), DES [38, 


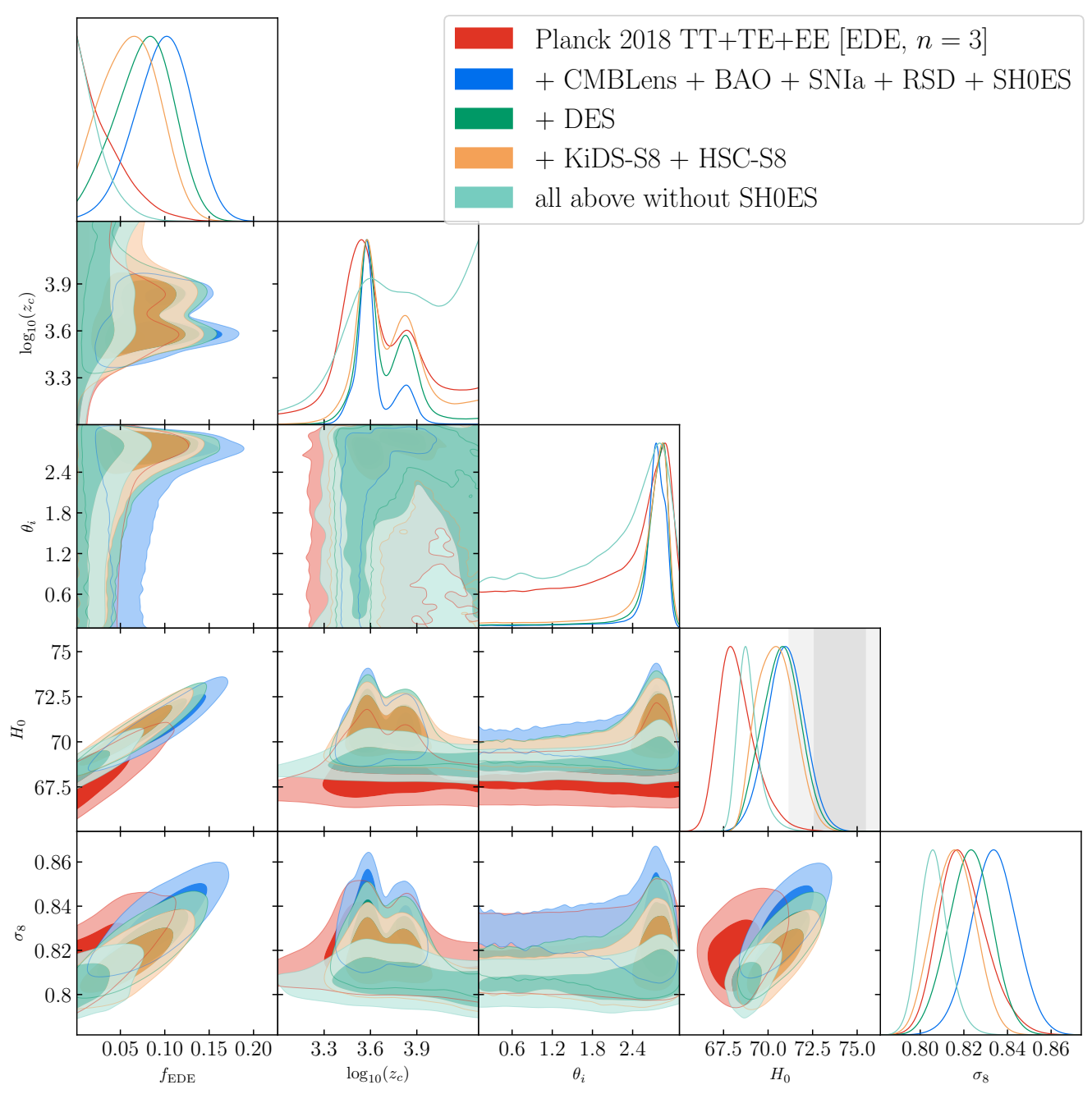

FIG. 1: Constraints on the EDE scenario from Planck 2018 primary CMB data (TT+TE+EE); Planck 2018 CMB lensing data; BAO data from 6dF, SDSS DR7, and SDSS DR12; Pantheon SNIa data; the latest SH0ES $H_{0}$ constraint; SDSS DR12 RSD data; and the DES-Y1 3x2pt data. Here we present a subset of the parameters: the EDE energy-density fraction, timing, and initial condition, denoted $f_{\mathrm{EDE}}, \log _{10}\left(z_{c}\right)$, and $\theta_{i}$, respectively (note $\theta_{i}$ is distinct from $\theta_{s}$, the latter being the angular size of the sound horizon), along with $H_{0}[\mathrm{~km} / \mathrm{s} / \mathrm{Mpc}]$ and $\sigma_{8}$. The contours show $1 \sigma$ and $2 \sigma$ posteriors for various data set combinations, computed with GetDist 37. The red contours show results for Planck primary CMB data alone; the blue contours additionally include Planck CMB lensing data, BAO data, SNIa data, SH0ES, and RSD data (matching the data sets used in [25] and 26], but with Planck 2018 replacing 2015); and the dark green contours further include the DES-Y1 3x2pt likelihood. The orange contours add priors on $S_{8}$ derived from KiDS and HSC data (as an approximation to the full likelihoods from these data sets). The Planck primary CMB data already place relatively strong constraints on the EDE scenario. Inclusion of the DES, KiDS, and HSC data significantly weakens the moderate evidence for EDE seen when analyzing the data sets used in 26. The $H_{0}$ increase found in the EDE model fit in 26] (blue contours here) is noticeably reduced by the inclusion of LSS data, and the tension with SH0ES (shown in the gray bands) is no longer reconciled. The light green contours include all data sets except SH0ES, yielding a stringent upper bound $f_{\mathrm{EDE}}<0.060$ at $95 \% \mathrm{CL}$, and a value for $H_{0}$ consistent with the fit to the primary CMB alone. Fig. 16 in Appendix A shows these constraints in terms of fundamental physics parameters.

the Kilo-Degree Survey (KiDS) [42, 43, and the Subaru Hyper Suprime-Cam (HSC) survey [44, amongst many others. Parallel to these new data sets, advances in the effective field theory of large-scale structure 45, 46 have allowed $\Lambda \mathrm{CDM}$ parameters to be precisely measured with the redshift-space galaxy power spectrum 11, 12, 47, in particular, a CMB-independent $1.6 \%$ measurement of the Hubble constant $H_{0}=68.6 \pm 1.1 \mathrm{~km} / \mathrm{s} / \mathrm{Mpc}$ [48. Pow- erful upcoming data sets from the Dark Energy Spectroscopic Instrument 49, Vera Rubin Observatory [50] (formerly LSST), Euclid [51, and WFIRST [52] are furthermore anticipated to significantly strengthen cosmological constraints.

In this work we reanalyze the EDE scenario taking into account Planck 2018 and DES-Y1 data in detail (in addition to many other data sets), as well as approximate 
Constraints on $\operatorname{EDE}(n=3)$ for varying data sets

\begin{tabular}{|c|c|c|c|c|c|}
\hline Parameter & $\begin{array}{c}\text { Planck } 2018 \\
\mathrm{TT}+\mathrm{TE}+\mathrm{EE}\end{array}$ & $\begin{array}{c}\text { Planck } 2018 \\
\text { TT+TE+EE, } \\
\text { CMB lensing, BAO, } \\
\text { RSD, SNIa, } \\
\text { and SHOES }\end{array}$ & $\begin{array}{c}\text { Planck } 2018 \\
\text { TT+TE+EE, } \\
\text { CMB lensing, BAO, } \\
\text { RSD, SNIa, } \\
\text { SH0ES, } \\
\text { and DES-Y1 }\end{array}$ & \begin{tabular}{|c|} 
Planck 2018 \\
TT+TE+EE, \\
CMB lensing, BAO, \\
RSD, SNIa, \\
SH0ES, \\
DES-Y1, \\
and HSC, KiDS $\left(S_{8}\right)$ \\
\end{tabular} & $\begin{array}{c}\text { Planck } 2018 \\
\text { TT+TE+EE, } \\
\text { CMB lensing, BAO, } \\
\text { RSD, SNIa, } \\
\text { DES-Y1, } \\
\text { and HSC, KiDS }\left(S_{8}\right) \\
\text { (no SH0ES) } \\
\end{array}$ \\
\hline $\begin{array}{l}f_{\mathrm{EDE}} \\
\log _{10}\left(z_{c}\right) \\
\theta_{i}\end{array}$ & $\begin{array}{c}<0.087 \\
3.66_{-0.24}^{+0.28} \\
>0.36\end{array}$ & $\begin{array}{c}0.098 \pm 0.032 \\
3.63_{-0.10}^{+0.17} \\
2.58_{-0.09}^{+0.29}\end{array}$ & $\begin{array}{c}0.077_{-0.032}^{+0.032} \\
3.69_{-0.15}^{+0.18} \\
2.58_{-0.15}^{+0.32}\end{array}$ & $\begin{array}{c}0.062_{-0.033}^{+0.032} \\
3.73_{-0.19}^{+0.20} \\
2.49_{-0.38}^{+0.40}\end{array}$ & $\begin{array}{l}<0.060 \\
>3.28 \\
>0.35\end{array}$ \\
\hline $\begin{array}{l}H_{0}[\mathrm{~km} / \mathrm{s} / \mathrm{Mpc}] \\
\sigma_{8}\end{array}$ & $\begin{array}{c}68.29_{-1.00}^{+1.02} \\
0.8198_{-0.0107}^{+0.0109} \\
\end{array}$ & $\begin{array}{c}70.98 \pm 1.05 \\
0.8337 \pm 0.0105\end{array}$ & $\begin{array}{c}70.75_{-1.09}^{+1.105} \\
0.8228_{-0.0101}^{+0.0099} \\
\end{array}$ & $\begin{array}{c}70.45_{-1.08}^{+1.05} \\
0.8157 \pm 0.0096\end{array}$ & $\begin{array}{c}68.92_{-0.59}^{+0.57} \\
0.8064 \pm 0.0065\end{array}$ \\
\hline
\end{tabular}

TABLE I: The mean $\pm 1 \sigma$ constraints on cosmological parameters in the EDE scenario (with index $n=3$, c.f. Eq. (11) from Planck 2018 primary CMB data (TT+TE+EE); Planck 2018 CMB lensing data; BAO data from 6dF, SDSS DR7, and SDSS DR12 (BOSS); Pantheon SNIa data; the latest SH0ES $H_{0}$ constraint; SDSS DR12 RSD data; and the DES-Y1 3x2pt data. Parameters in bold are sampled parameters. The two furthest-right columns add priors on $S_{8}$ derived from KiDS and HSC data (as an approximation to the use of full likelihoods from these data sets). In the furthest right column we include all data sets except the SH0ES measurement. Upper and lower limits are quoted at $95 \% \mathrm{CL}$; the one-sided $f_{\mathrm{EDE}}$ upper bounds for the +DES-Y1 (fourth column) and +DES-Y1+HSC+KiDS (fifth column) are $f_{\mathrm{EDE}}<0.127$ and $f_{\mathrm{EDE}}<0.112$ at $95 \%$ CL, respectively. The best-fit parameter values for most of these analyses can be found in Sec. VI The data set combination that yields the strongest evidence for EDE is shown in the third column (analogous to that used in 26]); the preferred EDE model in that analysis is in tension with the constraints on EDE imposed in the final column by the combination of all data sets without SH0ES, indicating discordance between SH0ES and the other data sets, even in the broadened EDE parameter space.

LSS constraints from KiDS and HSC. The DES-Y1 measurements are the most statistically powerful LSS data with publicly available likelihoods. We consider in detail the impact of EDE on the matter power spectrum and growth of structure, and the resulting constraints from LSS probes, in combination with CMB and cosmological distance information that has been used in previous EDE analyses.

The main results of this work are summarized in Fig. 1 and Table I. We find no evidence for EDE in Planck 2018 primary $\mathrm{CMB}$ data $(\mathrm{TT}+\mathrm{TE}+\mathrm{EE})$ alone, but instead find an upper bound $f_{\mathrm{EDE}}<0.087$ at $95 \%$ CL. In contrast, when considering the same data set combination as used in 25] and 26] (with CMB likelihoods updated to Planck 2018), consisting of the primary CMB supplemented by Planck 2018 CMB lensing data; BAO data from 6dF, SDSS DR7, and SDSS DR12; Pantheon SNIa data; the latest SHOES $H_{0}$ constraint; and SDSS DR12 RSD data; we find $3.1 \sigma$ evidence for EDE, consistent with past claims in the literature.

However, the inclusion of additional LSS data yields a downward trend in this result. The DES-Y1 3x2pt data bring the evidence for EDE down to $2.3 \sigma$. Interestingly, we find that the results of this computationally expensive analysis are extremely well approximated by a simple Gaussian prior on $S_{8}$ (see Sec. VID). Guided by this, we approximate HSC and KiDS data via priors on $S_{8}$, and find a further degradation of the evidence for EDE, $f_{\mathrm{EDE}}=0.062_{-0.033}^{+0.032}$, consistent with null at below $2 \sigma$ (orange contours in Fig. 1). The one-sided upper bound is $f_{\mathrm{EDE}}<0.112$ at $95 \% \mathrm{CL}$, and we constrain $H_{0}=70.45_{-1.08}^{+1.05} \mathrm{~km} / \mathrm{s} / \mathrm{Mpc}$. This constraint is in $2 \sigma$ tension with the SH0ES result on its own, shown by the gray bands in Fig. 1, indicating discordance.

To further assess the concordance of these varied data sets, we consider the fit to the combined data set including all likelihoods except SHOES. The results, shown as light green contours in Fig. 1, are statistically consistent with the fit to Planck 2018 primary CMB data $(\mathrm{TT}+\mathrm{TE}+\mathrm{EE})$ alone, and clearly inconsistent with SHOES. This analysis yields an even tighter upper bound on EDE, $f_{\mathrm{EDE}}<0.060$ at $95 \%$ CL, with $H_{0}=68.92_{-0.59}^{+0.57}$ $\mathrm{km} / \mathrm{s} / \mathrm{Mpc}$, in strong tension with SHOES.

Finally, we examine the choice of priors and the role of the axion decay constant. For computational efficiency, we limit ourselves to Planck 2018 primary CMB data $(\mathrm{TT}+\mathrm{TE}+\mathrm{EE})$ alone. We find that uniform priors imposed directly on the particle physics parameters $f$ and $\log _{10}(m)$ (see Eq. (11)) strongly downweight large $f_{\mathrm{EDE}}$ values, in comparison to uniform priors placed on the effective EDE parameters $f_{\mathrm{EDE}}$ and $\log _{10}\left(z_{c}\right)$. This is reflected in the posterior distributions, and in particular, that for $H_{0}$, which is a near identical match to that in $\Lambda \mathrm{CDM}$ (see Fig. 9).

The outline of this paper is as follows: in Sec. II we review the physics of the EDE proposal and its variants. In Sec. [III we describe our numerical implementation of the EDE model in a publicly available code, CLASS_EDE [53. In Sec. IV we study in detail the impact on LSS, particularly the matter power spectrum, and in Sec. $\mathrm{V}$, we discuss the data sets used in our analysis. We present our main results in Sec. VI followed by an examination of physical priors in Sec. VII and we conclude in Sec. VIII. Additional figures can be found in the Appendices. 


\section{THE EARLY DARK ENERGY PROPOSAL}

The goal of the EDE proposal is to allow for larger values of $H_{0}$ than obtained in $\Lambda \mathrm{CDM}$ when analyzing CMB power spectrum data, while not degrading the overall quality of the fit. This goal is achieved by demanding that the angular acoustic scale, namely the ratio of the sound horizon at last scattering to the comoving angular diameter distance to last scattering (at redshift $\left.z_{*} \approx 1100\right)$,

$$
\theta_{s}=\frac{r_{s}\left(z_{*}\right)}{D_{A}\left(z_{*}\right)}
$$

be unchanged by the new physics introduced to solve the tension. The acoustic scale is the best-measured quantity in CMB data: it is constrained to $0.03 \%$ precision in the Planck 2018 analysis, $100 \theta_{s}=1.0411 \pm 0.0003$ [5]. Upcoming CMB polarization data from Simons Observatory [54 and CMB-S4 [55] will independently constrain $\theta_{s}$ to this level (or better), providing a useful cross-check on the current CMB-temperature-dominated constraints.

The evolution of the Hubble parameter is encoded in $\theta_{s}$ via the integral expressions for $r_{s}$ and $D_{A}($ here $c=1$ ),

$$
r_{s}=\int_{z_{*}}^{\infty} \frac{\mathrm{d} z}{H(z)} c_{s}(z), D_{A}=\int_{0}^{z_{*}} \frac{\mathrm{d} z}{H(z)} .
$$

The former depends sensitively on $H(z)$ in the two decades of scale factor evolution prior to recombination, while the latter depends directly on $H_{0}$ (and low-redshift cosmic evolution). It follows that $\mathrm{a} \approx 10 \%$ increase in $H_{0}$, i.e., of order the early-universe discrepancy with lateuniverse measurements, can be compensated for in $\theta_{s}$ by $\mathrm{a} \approx 10 \%$ increase in $H(z)$ just prior to recombination.

A simple mechanism to realize this effect, while not disrupting the rest of CMB physics and the ensuing cosmological evolution, is to introduce an additional contribution to the cosmic energy budget, which constitutes $\approx 10 \%$ of the total energy density for a brief period just prior to recombination, and which rapidly decays away after achieving the required decrease in $r_{s}$. Thus, the new component acts as dark energy at early times, and then rapidly becomes irrelevant after a critical redshift where it decays. This early-time contribution to the cosmological constant is necessarily orders of magnitude greater than the present-day cosmological constant, $\rho_{\Lambda}^{1 / 4} \simeq \mathrm{meV}$. This hypothesized additional contribution is known as "early dark energy".

The simplest example of an effective cosmological constant which dynamically decays is that of a light scalar field. From the equation of motion of a scalar field $\phi$ with mass $m$ and potential $V(\phi)=m^{2} \phi^{2} / 2$,

$$
\ddot{\phi}+3 H \dot{\phi}+m^{2} \phi=0,
$$

one can see that if initially $m \ll H$, then Hubble friction will freeze $\phi$ at its initial value $\phi_{i}$, contributing a vacuum energy $m^{2} \phi_{i}^{2} / 2$ to the cosmological constant. Once the
Hubble parameter drops below the mass, $m \gg H$, the field begins to oscillate, $\phi(t)=\phi_{i} a^{-3 / 2} \cos (m t)$, and the vacuum energy redshifts away as matter $\left(\propto a^{-3}\right)$.

To utilize such a field to resolve the Hubble tension, the field must begin to oscillate in the rough ballpark of $z_{\mathrm{CMB}}$, at which point the Hubble parameter $H \sim T^{2} / M_{p l}$ is roughly $10^{-27} \mathrm{eV}^{2}$ Thus the scalar field in question must be extremely light. From a particle physics standpoint, the only known example of such a field is the axion [56 58.

The axion is defined as a (pseudo)-scalar endowed with a global $U(1)$ shift symmetry, broken by non-perturbative effects, namely instantons, that generate a potential (see, e.g., [59]),

$$
V(\phi)=\sum_{n} c_{n} e^{-S_{n}} \cos (n \phi / f) \simeq V_{0} \cos \frac{\phi}{f}+\ldots
$$

breaking the continuous shift symmetry to a discrete shift symmetry. This shift symmetry protects the axion mass from radiative corrections, allowing for extremely small values of the axion mass. The ... in the above equation indicates subdominant instantons, exponentially suppressed by the charge- $n$ instanton action $S_{n}$. Gravitational instantons scale as $S_{n} \simeq n M_{p l} / f$ [59, 60]. If $f>M_{p l}$, the instanton expansion breaks down and the potential cannot be approximated by the leading term [61, 62].

To resolve the Hubble tension, the leading-order instanton will not suffice. The EDE field must rapidly decay away so as to leave low-redshift cosmic evolution unchanged, while the axion redshifts only as matter. Thus, its effects would spoil late-time cosmology. The proposal of [25] is then to consider potentials of the form (e.g., [34),

$$
V=V_{0}(1-\cos (\phi / f))^{n}, V_{0} \equiv m^{2} f^{2},
$$

corresponding to a careful fine-tuning of the hierarchy of instanton actions. For integer values of $n$, this finetuning is limited to the first $n$ terms in the expansion in Eq. (4). For arbitrary real-valued $n$, one must instead fine-tune an infinite tower of terms. For this reason, we will restrict our analysis to integer values of $n$ (primarily $n=3)$.

The minimum of the potential (5) is locally $V \sim \phi^{2 n}$, in which case the oscillations of $\phi$ correspond to an equation of state 63,

$$
w_{\phi}=\frac{n-1}{n+1} .
$$

For $n=2$, the initial energy stored in the field (i.e., the EDE) redshifts away during the oscillatory phase as

\footnotetext{
${ }^{2}$ We denote the reduced Planck mass as $M_{p l}=2.435 \times 10^{18} \mathrm{GeV}$ here and throughout.
} 


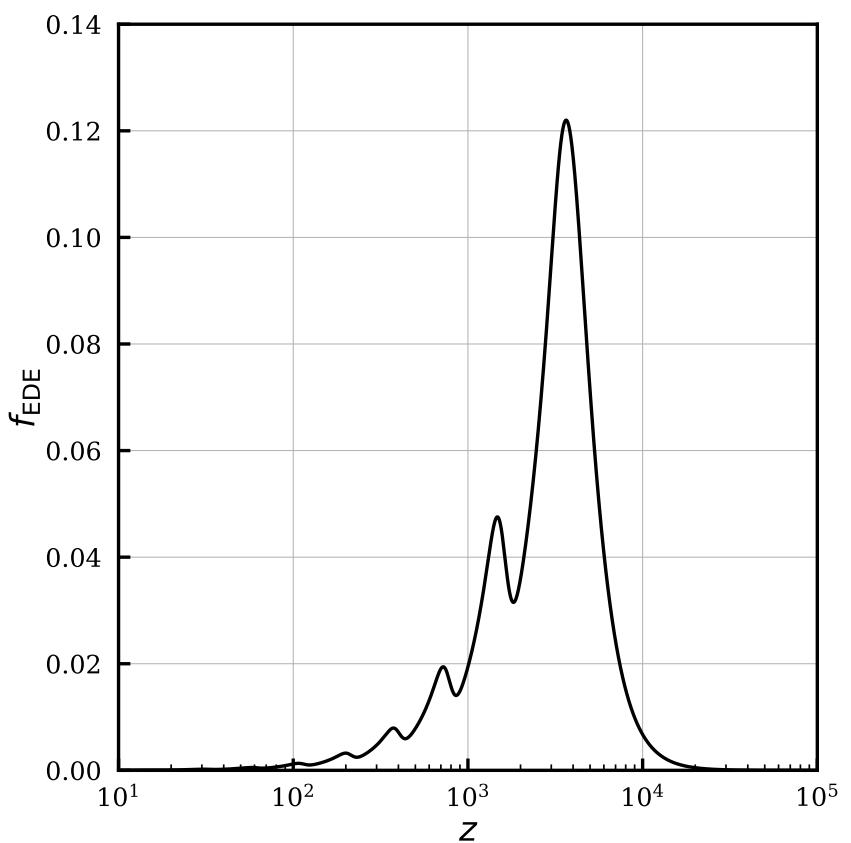

FIG. 2: Fraction of the cosmic energy density in the EDE field $\phi$ as a function of redshift, for the parameters in Eq. (7).

radiation $\left(\propto a^{-4}\right)$, and for $n \rightarrow \infty$ it redshifts as kinetic energy $\left(\propto a^{-6}\right)$.

These dynamics allow the model to be succinctly described in terms of an initial field displacement $\theta_{i} \equiv \phi_{i} / f$, and two effective parameters, $z_{c}$ and $f_{\mathrm{EDE}}$, which are defined by the redshift $z_{c}$ at which the EDE makes its maximal fractional contribution $f_{\mathrm{EDE}}$ to the total energy density of the universe, $\left.f_{\mathrm{EDE}}\left(z_{c}\right) \equiv\left(\rho_{\mathrm{EDE}} / 3 M_{p l}^{2} H^{2}\right)\right|_{z_{c}} \cdot{ }^{3}$ The dependence of $f_{\mathrm{EDE}}$ and $z_{c}$ on the particle physics parameters $m$ and $f$ and the initial angle $\theta_{i}$ is highly non-linear, a fact that we will return to in Sec. VII

As a numerical example, we consider $n=3, f_{\mathrm{EDE}}=$ $0.122, \log _{10}\left(z_{c}\right)=3.562$, and $\theta_{i}=2.83$. This is the bestfit integer- $n$ model reported in the [26] fit to CMB, CMB lensing, BAO, redshift-space distortion (RSD), SNIa, and SH0ES data. We will refer to this example throughout; the full set of parameters is given by

$$
\begin{array}{rlrl}
H_{0} & =72.19 \mathrm{~km} / \mathrm{s} / \mathrm{Mpc}, & 100 \omega_{b}=2.253 \\
\omega_{\mathrm{cdm}} & =0.1306, & 10^{9} A_{s}=2.215, \\
n_{s} & =0.9889, & \tau_{\text {reio }}=0.072 \\
f_{\mathrm{EDE}} & =0.122, \log _{10}\left(z_{c}\right)=3.562, \theta_{i}=2.83 .
\end{array}
$$

The resulting evolution of the EDE component is shown in Fig. 2 At its peak (near $z \approx 3650$ ), the EDE field comprises $12 \%$ of the energy density of the universe. This

\footnotetext{
${ }^{3}$ For notational simplicity, $f_{\mathrm{EDE}}\left(z_{c}\right)$ will be denoted as $f_{\mathrm{EDE}}$ in most contexts.
}

is then rapidly dissipated as the field starts to roll and oscillate, and at $z=10^{3}$ its contribution is less than $2 \%$ of the energy density of the universe.

A minimal alternative to power-law cosine potentials is to consider instead the only aspect seemingly relevant to the Hubble tension, namely the shape of the potential at the minimum. This is the approach of [27], who studied

$$
V=V_{0}\left(\frac{\phi}{M_{p l}}\right)^{2 n}
$$

This coincides with the earlier models for small initial field values $\phi_{i} / f \ll 1$. The most recent statistical analysis [26] found such monomial potentials are disfavoured relative to the cosine potential with a large initial field displacement.

There are now many realizations of the EDE scenario that have been proposed. Unstable dark energy, a.k.a "Axion-Dilaton Destabilization" 28, is a realization of EDE without higher-order instantons. This is done with a two-field model:

$$
V(\phi, \chi)=\frac{1}{2} m_{\chi}^{2} f^{2} e^{\beta(\phi)}\left(1+\cos \frac{\chi}{f}\right)+V_{0} e^{-\lambda \phi / M_{p l}},
$$

where $\beta(0)>0$. The axion $\chi$ rolls down its potential at a time $z_{c}$, triggering the destabilization of a second scalar field $\phi$ with a steep potential, $\lambda \gg 1$. The two-field model 28 can be qualitatively understood by considering a single-field truncation, with a piece-wise-defined potential for the EDE field, in a manner resembling the best-fit "Acoustic Dark Energy" of [29. Other EDElike possibilities include a "kick" from neutrino freeze-out 30, a first-order phase transition 31, parametric resonance [32], and dissipation into gauge fields [33]. For this work, we will concentrate on the cosine potentials as proposed in 25], which have been shown to fit cosmological data well and serve as a canonical example of the EDE scenario.

The hallmark success of this proposal lies in preserving the fit to the Planck CMB temperature power spectrum. The best-fit EDE model analyzed in $\left[26\right.$ has $H_{0} \approx 72$ $\mathrm{km} / \mathrm{s} / \mathrm{Mpc}$, while leaving no visible imprint on the CMB compared to a $\Lambda \mathrm{CDM}$ model with significantly lower $H_{0}$. To illustrate this, we show the CMB temperature power spectra in $\Lambda \mathrm{CDM}$ with $H_{0} \approx 68 \mathrm{~km} / \mathrm{s} / \mathrm{Mpc}$ and in a fiducial EDE model with $H_{0} \approx 72 \mathrm{~km} / \mathrm{s} / \mathrm{Mpc}$, in Fig. 3 . Analogous figures for the $\mathrm{CMB}$ polarization and lensing power spectra (including the fractional change with respect to $\Lambda \mathrm{CDM}$ ) can be found in Appendix D (see Figs. 21 and 22). The model parameters for these figures are chosen as the best-fit values reported in [26]: EDE with parameters as in Eq. 77), and $\Lambda$ CDM with parameters given by

$$
\begin{array}{rlrl}
H_{0} & =68.21 \mathrm{~km} / \mathrm{s} / \mathrm{Mpc} & 100 \omega_{b} & =2.253, \\
\omega_{\mathrm{cdm}} & =0.1177 & 10^{9} A_{s} & =2.216, \\
n_{s} & =0.9686 & \tau_{\text {reio }} & =0.085 .
\end{array}
$$



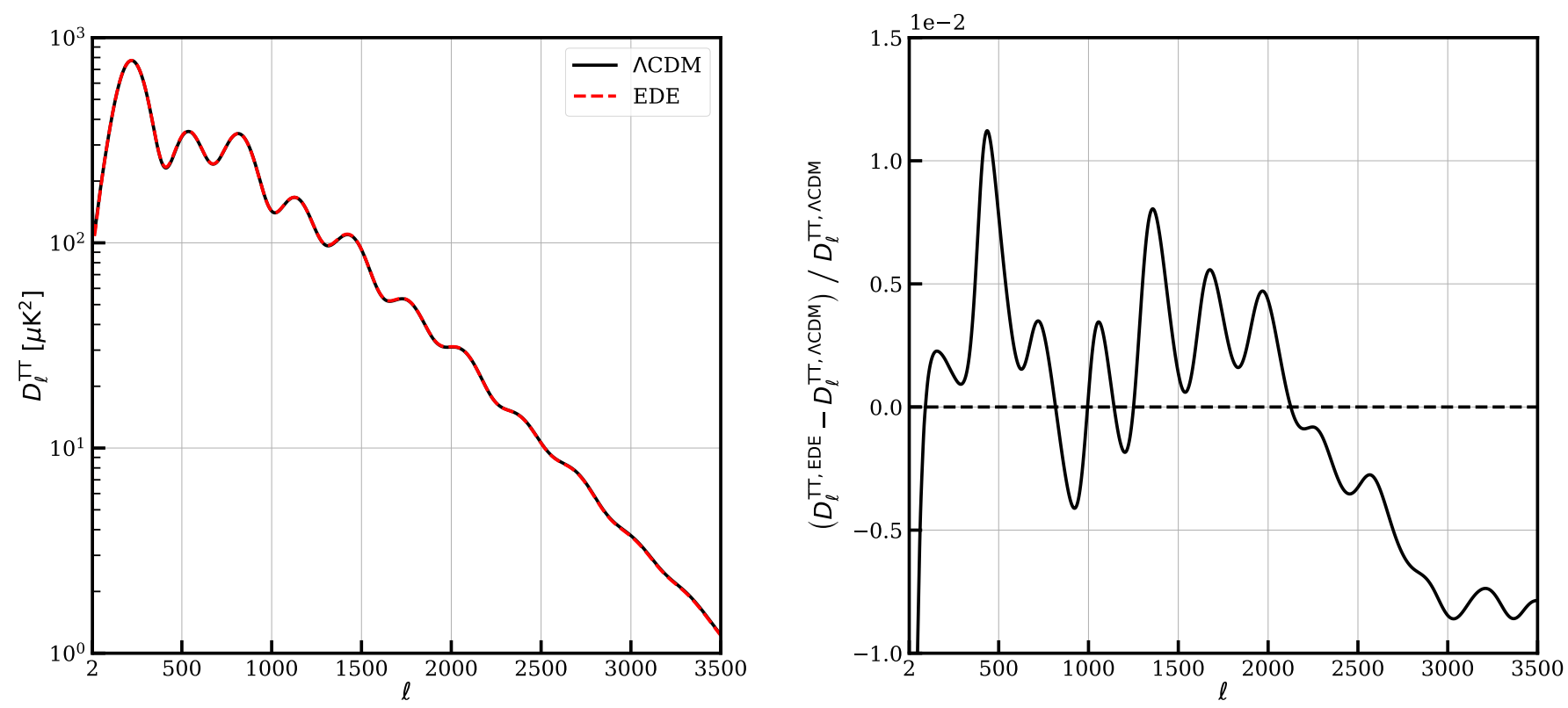

FIG. 3: CMB temperature anisotropy power spectra (left panel) and residuals (right panel) for $\Lambda$ CDM (black, solid) and EDE (red, dashed) models, with $H_{0}=68.21 \mathrm{~km} / \mathrm{s} / \mathrm{Mpc}$ and $H_{0}=72.19 \mathrm{~km} / \mathrm{s} / \mathrm{Mpc}$, respectively. The curves are nearly indistinguishable in the left panel. The model parameters are given by Eqs. (7) and 10 for EDE and $\Lambda$ CDM, respectively, corresponding to the [26] best-fit models to primary CMB, CMB lensing, BAO, RSD, SNIa, and SH0ES data.

Figs. 3 and 22 show that the primary CMB power spectra are nearly indistinguishable in these two models, despite the EDE model having a significantly larger $H_{0}$ than the $\Lambda \mathrm{CDM}$ model. This suggests that EDE can provide a simple early-universe solution to the Hubble tension.

\section{NUMERICAL IMPLEMENTATION}

We implement the EDE scenario as a modification to the publicly available Einstein-Boltzmann code CLASS 64, 65..$^{4}$ Our modified version, CLASS_EDE, is publicly available $53 .{ }^{5}$ CLASS_EDE solves for the evolution of the scalar field perturbations directly using the perturbed Klein-Gordon equation (as in, e.g., [27] and [26]), avoiding the effective fluid description used in some past works (e.g., 25]). We implement adiabatic initial conditions for the scalar field fluctuations as described in [26; see 26] for a discussion of isocurvature initial conditions. The EDE potential is implemented as

$$
V(\phi)=m^{2} f^{2}(1-\cos (\phi / f))^{n}+V_{\Lambda},
$$

where $V_{\Lambda}$ is a constant, which plays the role of the cosmological constant. Absorbing the cosmological constant into $V(\phi)$ allows efficient closure of the energy budget equation, $\sum \Omega_{i}=1$ in a flat universe, for arbitrary model

\footnotetext{
4 http://class-code.net

5 https://github.com/mwt5345/class_ede
}

parameters simply by tuning $V_{\Lambda}$ via the built-in shooting functionality of CLASS.

CLASS_EDE allows one to specify the EDE model parameters in terms of the particle physics parameters $f$ and $m$ or the effective EDE parameters $f_{\mathrm{EDE}}$ and $z_{c}$. If the latter set is specified, CLASS_EDE will find the corresponding $f$ and $m$ via a shooting algorithm, analogous to the shooting of $H_{0}$ from a user-specified $100 \theta_{s}$ in CLASS. For both implementations the user must also specify the initial axion misalignment angle $\theta_{i} \equiv \phi_{i} / f$ and a value for $n$. To handle dynamics for small values of $f_{\mathrm{EDE}}$, we have increased the default time-step precision in CLASS to $7 \times 10^{-4}$. The final update to the functionality of CLASS is a simple extraction of $f \sigma_{8}(z)$, where here $f \equiv d \log D / d \log a$ is the linear growth rate (not the axion decay constant), which is needed to implement the RSD likelihoods in our analysis below. In all likelihoods requiring calculations of the non-linear matter power spectrum, we compute this quantity using the "Halofit" prescription implemented in CLASS [66, 67].

We perform Markov chain Monte Carlo (MCMC) analyses using the publicly available code Cobaya 68. We sample from the posterior distributions using the Metropolis-Hastings algorithm implemented in Cobaya 69 71, with a Gelman-Rubin [72] convergence criterion $R-1<0.05$. To determine best-fit parameter values, we use the "BOBYQA" likelihood maximization method implemented in Cobaya 73 75. 


\section{IMPRINT ON LARGE-SCALE STRUCTURE}

The introduction of the EDE field into the cosmological model affects observables beyond the CMB temperature and polarization power spectra. In particular, it affects the dynamics of all perturbation modes that are within the horizon during the epoch in which the EDE is relevant. This change in the transfer function leaves signatures in the late-time matter power spectrum $P(k)$. Moreover (and more significantly), the "standard" cosmological parameters must shift in the EDE scenario in order to maintain the fit to the primary $\mathrm{CMB}$ data while accommodating a higher $H_{0}$ value than possible in $\Lambda \mathrm{CDM}$. These shifts, particularly in $\omega_{\text {cdm }}$, significantly affect $P(k)$. As precise measurements of this observable are available from current surveys (e.g., [38, 41, 42, 44]) and will dramatically grow in precision with near-future surveys (e.g., 49 52), it is an opportune time to examine their role in constraining the EDE scenario.

Following general conventions, we will often quantify LSS constraints by the $\sigma_{8}$ parameter, i.e., the RMS linear-theory mass fluctuation in a sphere of radius $8 \mathrm{Mpc} / h$ at $z=0$. This is evaluated in Fourier space as an integral over the matter power spectrum with a spherical top-hat filter $W(k R)$ of radius $R=8 \mathrm{Mpc} / h$, i.e.,

$$
\left(\sigma_{8}\right)^{2}=\frac{1}{2 \pi^{2}} \int \mathrm{d} \log k W^{2}(k R) k^{3} P(k) .
$$

The value of $\sigma_{8}$ is predominantly determined by contributions to the integral from $0.1 h / \mathrm{Mpc} \lesssim k \lesssim 1 h / \mathrm{Mpc}$, due to a balancing of high- $k$ suppression of the filter and the small- $k$ suppression from the $k^{3}$ factor.

In recent years, CMB observations have consistently yielded best-fit values of $\sigma_{8}$, or similarly $S_{8} \equiv$ $\sigma_{8}\left(\Omega_{m} / 0.3\right)^{0.5}$, that are slightly greater than those found by LSS observations. In the fit to $\Lambda$ CDM, the Planck 2018 analysis finds $S_{8}=0.830 \pm 0.013$ [5], while the DESY1 3x2pt analysis finds $S_{8}=0.773_{-0.020}^{+0.026}[38$, KiDS finds $S_{8}=0.745 \pm 0.039$ [42], and HSC finds $S_{8}=0.780_{-0.033}^{+0.030}$ [44]. Taken in conjunction as three independent measurements and combined with inverse-variance weights, these LSS experiments yield $S_{8}=0.770_{-0.016}^{+0.018}$, in $2.7 \sigma$ tension with the Planck 2018 CMB result.

This tension is worsened in the EDE scenario. For example, the [26] results for the best-fit integer- $n$ EDE model give $S_{8}=0.842 \pm 0.014$, increasing the tension with the LSS result quoted above to $3.2 \sigma$. Moreover, $S_{8}$ is only a single number, while LSS data constrain the shape of $P(k)$ over a decade in $k$-space. The value of $S_{8}$ depends on multiple $\Lambda$ CDM parameters, which are shifted in the EDE scenario in order to maintain the fit to the CMB acoustic peaks and the damping tail of the power spectrum. The upward shift of $S_{8}$ is predominantly driven by the increase in the physical CDM density, which slightly shifts the peak of the matter power spectrum and increases the growth rate of perturbations at late times. To illustrate this effect, in Fig. 4 we plot the non-linear matter power spectrum, computed with the "Halofit" prescription implemented in CLASS 66, 67, which is used in our analysis of LSS data in Sec. VI. The increase in power at $0.1 h / \mathrm{Mpc} \lesssim k \lesssim 1 h / \mathrm{Mpc}$ leads to an increase in $\sigma_{8}$ and $S_{8}$ (although these quantities are of course computed from the linear rather than non-linear power spectrum), and thus a worsening of the tension between these CMB-derived predictions and actual LSS data. We emphasize that this primarily arises from the change to the $\Lambda \mathrm{CDM}$ parameters in the EDE scenario, as is required to produce CMB power spectra that match the Planck fit to $\Lambda$ CDM (see Fig. 3).

On large scales $\left(k \lesssim 10^{-2} h / \mathrm{Mpc}\right)$, outside the reach of current LSS experiments, the $\operatorname{EDE} P(k)$ is suppressed relative to that in $\Lambda \mathrm{CDM}$. This difference is driven by the slight change in the primordial spectral index, with $n_{s}=$ 0.9889 and $n_{s}=0.9686$ in EDE and $\Lambda$ CDM, respectively, while the amplitude at the pivot scale $k_{\text {piv }} \equiv 0.05 \mathrm{Mpc}^{-1}$ remains essentially unchanged $\left(A_{s}=2.215 \times 10^{-9}\right.$ and $A_{s}=2.216 \times 10^{-9}$ in EDE and $\Lambda \mathrm{CDM}$, respectively).

These differences persist across redshift: in Fig. 5 we show the ratio of $P(k)$ in the two scenarios at $z=0$ (i.e., the ratio of the curves in Fig. 4 and at the midpoints of the DES-Y1 redshift bins. From this, one can see that the change in the matter power spectrum is substantial, up to $\approx 10 \%$ for a wide range of wavenumbers that are wellmeasured in current data. The figure also shows that the quasi-linear and small-scale changes are more significant at higher redshift.

The redshift dependence of the deviations from $\Lambda \mathrm{CDM}$ are also encoded in the growth factor, $f(z)$, as well as $f \sigma_{8}(z)$. We include plots of these quantities in Appendix $\mathrm{D}$ in Figs. 23 and 24 . The enhancement in EDE of $f \sigma_{8}$ at $z=1$ is twice that at $z=0$, given by $3 \%$ and $1.5 \%$, respectively. In comparison, BOSS RSD data provide a $6 \%$ measurement of $f \sigma_{8}$ at $z=0.38,0.51$, and 0.61 [41. Upcoming measurements from DESI will significantly improve upon these constraints 49 .

The $\sigma_{8}$ change does not fully capture the rich impact of EDE on the matter power spectrum. The effects of the EDE field modify the dynamics of all modes within the horizon (or those that re-enter the horizon) during the epoch in which the EDE makes a non-negligible contribution to the cosmic energy budget, i.e., around $z_{c}$ (with a wider redshift window for larger $\left.f_{\mathrm{EDE}}\right)$. These effects are sensitive to the amount and timing of EDE, as parameterized by $f_{\mathrm{EDE}}$ and $z_{c}$. The imprint of $f_{\mathrm{EDE}}$ on $P(k)$ can be seen in Fig. 6, while holding $z_{c}$ and $\theta_{i}$ fixed. Similarly, in Fig. 7 we show the matter power spectrum as a function of $\log _{10}\left(z_{c}\right)$, with $f_{\mathrm{EDE}}$ and $\theta_{i}$ held fixed. In both cases the $\Lambda \mathrm{CDM}$ parameters are also held fixed (to their values in Eq. (7)).

These figures show that $f_{\mathrm{EDE}}$ acts to suppress structure on small scales, with an effect that is compounded for small values of $z_{c}$, that is, models in which the EDE is relevant in the late universe. Physically, this is due to the suppression of perturbation growth by the accelerated expansion, analogous to (but weaker than) that in late-time 


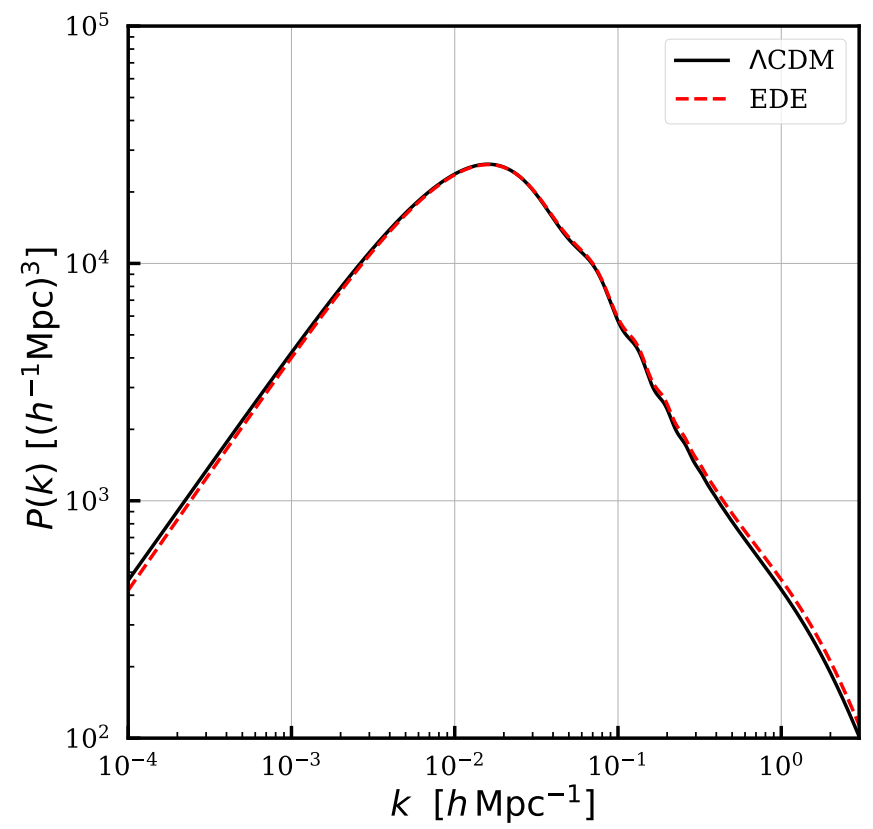

FIG. 4: Non-linear matter power spectrum $P(k)$ at $z=0$ for $\Lambda \mathrm{CDM}$ and EDE models that fit the primary CMB, distances, and SH0ES data. The change in $\sigma_{8}$ in the EDE scenario can be seen as the relative increase in $P(k)$ in the range $0.1 h / \mathrm{Mpc} \lesssim k \lesssim 1 h / \mathrm{Mpc}$ (although $\sigma_{8}$ is computed from the linear rather than non-linear power spectrum). This increase is due primarily to shifts in the "standard" cosmological parameters in the EDE model, rather than the effects of the EDE itself. The model parameters are the same as in Fig. 3 (see Eqs. 77) and (10).

dark energy domination. Quantitatively, we confirm this intuition by computing the wavenumber $k_{c}$ corresponding to the size of the comoving horizon at $z_{c}$, when the EDE has maximal influence on the dynamics. For the fiducial model considered in this section with $\log _{10}\left(z_{c}\right)=3.562$, we find $k_{c} \approx 0.03 h / \mathrm{Mpc}$. Fig. 6 clearly shows increasing suppression for modes with $k>k_{c}$ as $f_{\mathrm{EDE}}$ increases, which makes sense as these modes are all within the horizon at that time. There is also some suppression for modes with slightly lower $k$, as these modes re-enter the horizon while the EDE is still a non-negligible contribution to the cosmic energy budget.

Finally, to contextualize the EDE impact on LSS, we consider a comparison between the matter power spectrum in EDE and a model consistent with DES-Y1 measurements of photometric galaxy clustering, galaxygalaxy lensing, and cosmic shear two-point correlation functions 38. The latter yield constraints $S_{8}=$ $0.773_{-0.020}^{+0.026}$ and $\Omega_{m}=0.267_{-0.017}^{+0.030}$. The DES measurements are generally insensitive to the other $\Lambda \mathrm{CDM}$ parameters, and we adopt Planck 2018 [5] TT+TE+EE best-fit values $n_{s}=0.9649, h=0.6727, \tau_{\text {reio }}=0.0544$, and $\Omega_{b} h^{2}=0.02237$ to complete the model. The amplitude $A_{s}$ is set by CLASS to reproduce the DES measurement of $\sigma_{8}$, which gives $A_{s}=2.788 \times 10^{-9}$. We consider a

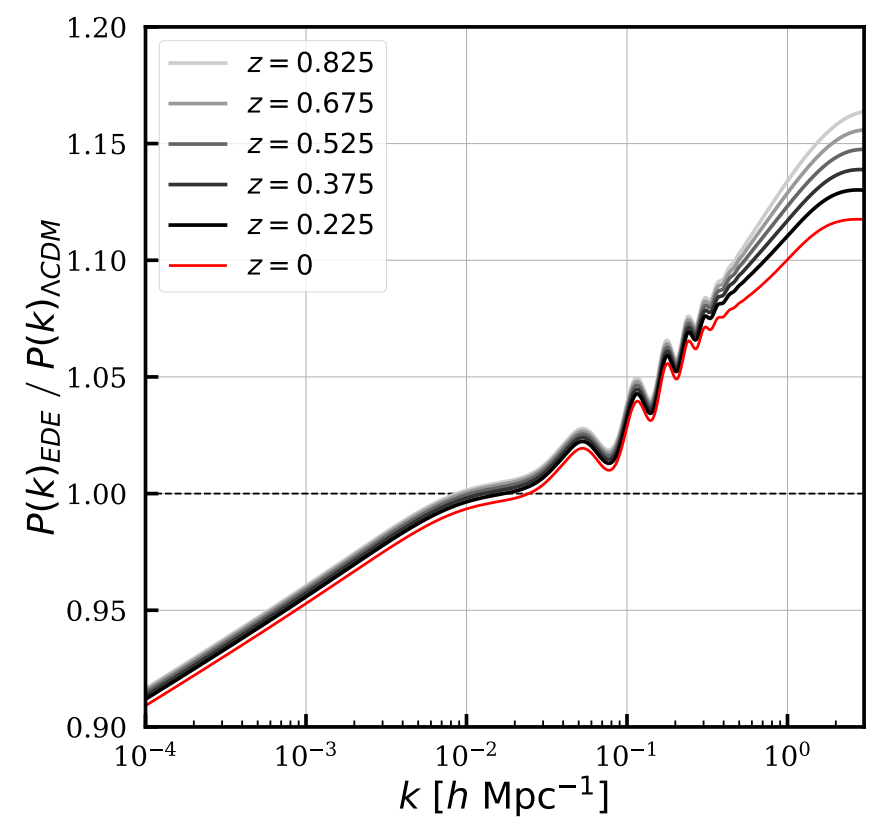

FIG. 5: Ratio of the EDE and $\Lambda$ CDM non-linear matter power spectra at $z$ values chosen to be the midpoints of the redshift bins used in the DES-Y1 analysis (and at $z=0$ in red). The model parameters are the same as in Fig. 3 .

redshift $z=0.525$, corresponding to the central redshift bin of DES.

A comparison of $P(k)$ in the best-fit EDE and $\Lambda \mathrm{CDM}$ models of [26, Eqs. (7) and (10), and the model consistent with DES-Y1 is shown in Fig. 8 . The blue shaded region corresponds to the approximate range of comoving wavenumbers probed the DES angular correlation functions, which span the range $2.5^{\prime}<\theta<250^{\prime}$, with a lower scale cut imposed at comoving separations $R \approx 2$ $12 \mathrm{Mpc} / h$, depending on the observable. In particular, the right panel of Fig. 8 displays the ratio of $P(k)$ predicted by the EDE model to that inferred by DES in $\Lambda \mathrm{CDM}$; this shows an even greater suppression of power on large scales than in Fig. 4, and an even greater enhancement on small scales. The enhancement on small scales can again be understood in terms of the physical CDM density $\Omega_{c} h^{2}$, which is $\Omega_{c} h^{2}=0.0984$ for DES (with $h$ and $\Omega_{b} h^{2}$ fixed by Planck), but $\Omega_{c} h^{2}=0.1306$ in the EDE model.

The suppression on large scales, which is beyond the observable range of DES or other current surveys, is driven by the significant shift in $A_{s}$, enhanced by the shift in $n_{s}$, and to a lesser extent by the significant shift in the matter density $\Omega_{m}$, which is $\Omega_{m}=0.267$ for DES and $\Omega_{m}=0.303$ for the EDE model parameters in Eq. (7).

\section{DATA SETS}

We consider the following data sets in our MCMC analyses: 


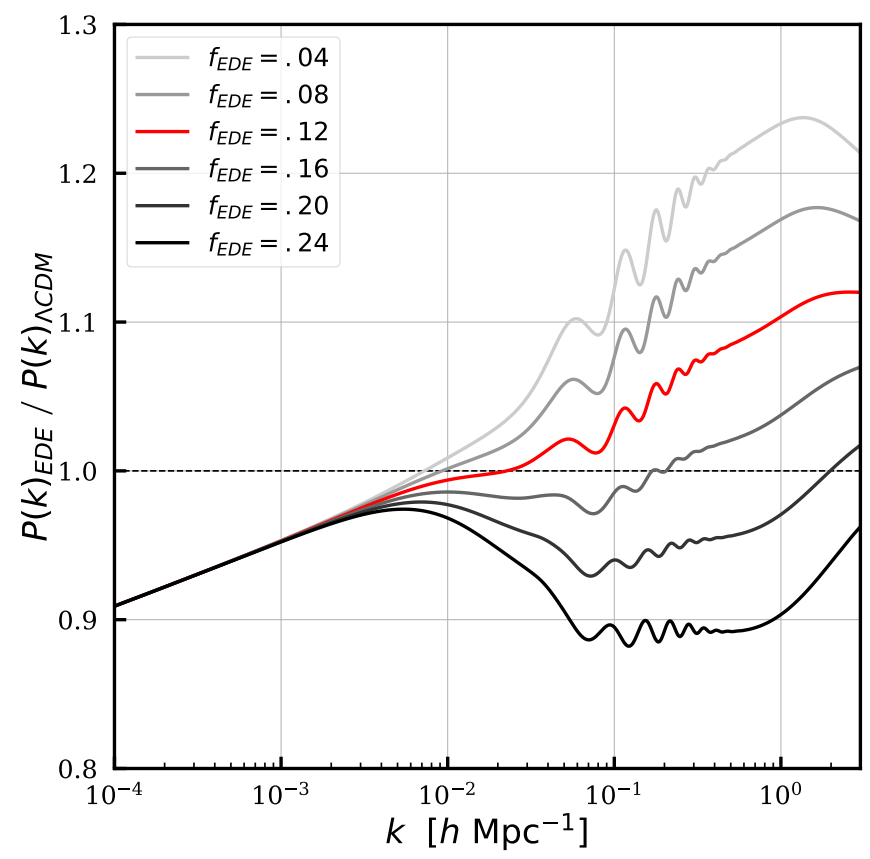

FIG. 6: Ratio of $P(k)$ in EDE to that in $\Lambda$ CDM as a function of the EDE fraction $f_{\mathrm{EDE}}$, at fixed $\log _{10} z_{c}=3.526$ and $\theta_{i}=2.83$. The other model parameters are given in Eq. (7). As $f_{\mathrm{EDE}}$ increases, the growth of perturbations that are within the horizon during the EDE epoch is suppressed by a progressively greater amount. The red curve here is identical to that shown in Fig. 5 .

\section{A. CMB}

Planck 2018 [5, 76, 77] low- $\ell$ and high- $\ell$ [Plik] temperature, polarization, and $\mathrm{CMB}$ lensing power spectra. In comparison with the Planck 2015 results [4, 78], the primary change in the fit to $\Lambda C D M$ is a shift in the mean value and tightening in the error bar on the optical depth to reionization, from $\tau_{\text {reio }}=0.066 \pm 0.016$ in 2015 to $\tau_{\text {reio }}=0.054 \pm 0.007$ in 2018, as well as a small shift downward of $n_{s}$ and a small shift upward in $\omega_{\text {cdm }}$.

\section{B. LSS}

In addition to the Planck 2018 CMB lensing data set [7], which we consider to be an LSS data set as it probes the low-redshift universe, we include:

BAO: Measurements from the SDSS DR7 main galaxy sample [79] and the $6 \mathrm{dF}$ galaxy survey [80] at $z=0.15$ and $z=0.106$, respectively, as well as from the SDSS BOSS DR12 41] optimally combined LOWZ and CMASS galaxy samples at $z=0.38,0.51$, and 0.61 .

RSD: SDSS BOSS DR12 41, 81] measurements of $f \sigma_{8}(z)$ from the imprint of peculiar velocities on the conversion between configuration- and redshift-space

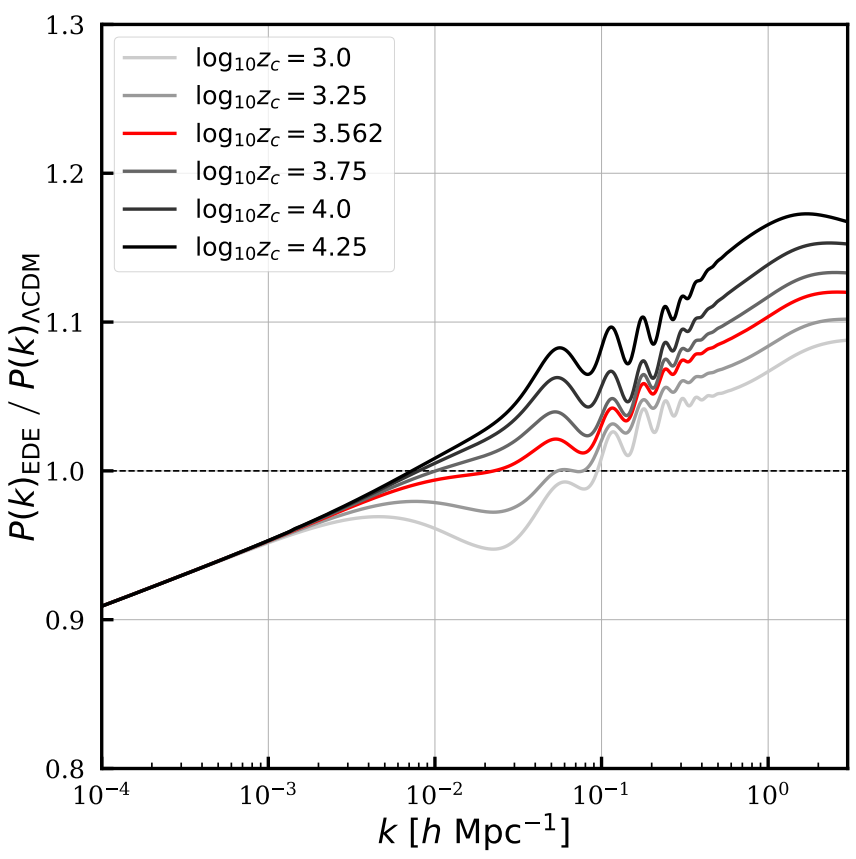

FIG. 7: Ratio of $P(k)$ in EDE to that in $\Lambda$ CDM as a function of the critical redshift $\log _{10} z_{c}$, at fixed $f_{\mathrm{EDE}}=0.12$ and $\theta_{i}=$ 2.83. The other model parameters are given in Eq. 77. As the critical redshift decreases, the growth suppression due to EDE is pushed to progressively later times, and thus affects modes on correspondingly larger scales (lower $k$ ).

82 , at $z=0.38,0.51$, and 0.61 . We include the full covariance of the joint BOSS DR12 BAO and RSD data. In particular, we use the "consensus" final $\mathrm{BAO}+\mathrm{RSD}$ results given in 41 in their Table 7 (final column) and the covariance given in their Table $8 .^{6}$

DES: shear-shear, galaxy-galaxy, and galaxy-shear two-point correlation functions (often referred to as "3x2pt"), measured from 26 million source galaxies in four redshift bins and 650,000 luminous red lens galaxies in five redshift bins, for the shear and galaxy correlation functions, respectively 38. When analyzed in $\Lambda \mathrm{CDM}$, the DES $3 \times 2$ pt likelihood gives tight constraints on $S_{8}$ and $\Omega_{m}, S_{8}=0.773_{-0.020}^{+0.026}$ and $\Omega_{m}=0.267_{-0.017}^{+0.030}$, respectively.

Additional LSS data: Weak gravitational lensing measurements from KiDS+VIKING-450 (hereafter KiDS or

\footnotetext{
${ }^{6}$ We note that, at the time our analysis was performed, there was a non-negligible bug in the implementation of the BOSS DR12 BAO+RSD likelihood in the Monte Python MCMC sampling code, which has afflicted previous EDE analyses in the literature (https://github.com/brinckmann/montepython public/issues/112). We have explicitly checked that this bug is not present in Cobaya, and that Cobaya reproduces the $\chi^{2}$ values for this likelihood found in the official Planck 2018 MCMC chains.
} 

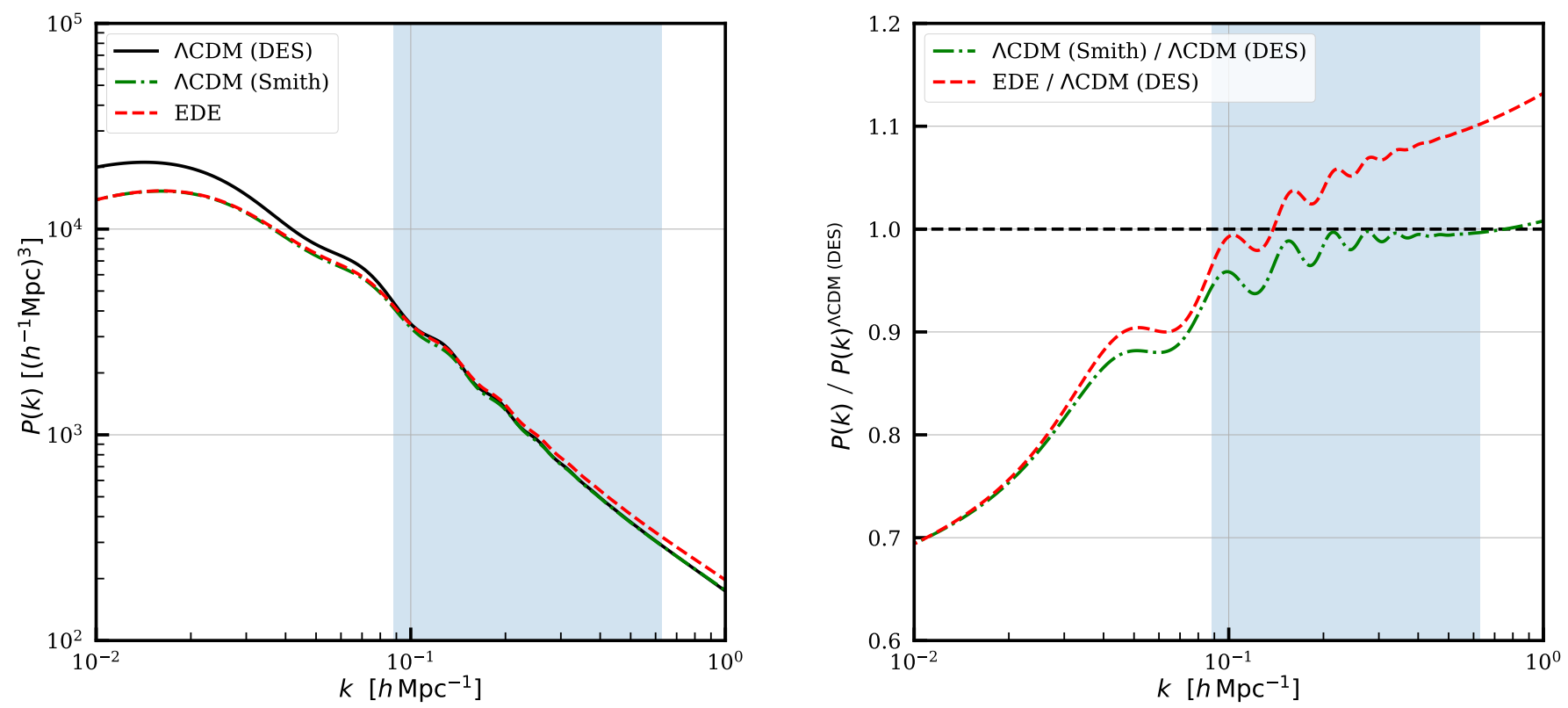

FIG. 8: Matter power spectra (left panel) and ratio of spectra (right panel) at the central redshift of DES observations, $z=0.525$, for $\Lambda$ CDM with the DES-Y1 best-fit values $\Omega_{m}=0.267$ and $S_{8}=0.773$ (black, solid), the best-fit EDE model from 26 with parameters given in Eq. 77) (red, dashed), and the best-fit $\Lambda$ CDM model from 26] with parameters given in Eq. 10 (green, dash-dotted). The ratios in the right panel are computed with respect to DES-Y1 $\Lambda$ CDM, thus giving an indication of how well the other two models' predictions match the DES-Y1 constraints. The blue shaded region is the approximate range of comoving wavenumbers probed by the angular scale cuts used in the DES-Y1 analysis.

KV-450) 42, 43, and the Subaru Hyper Suprime-Cam (HSC) [44, which provide complementary data sets to the Dark Energy Survey, and impose similar (though slightly weaker) constraints on $S_{8}$ and $\Omega_{m}$. We do not include likelihoods for these datasets directly, but we approximately include their effect via priors on $S_{8}$. For KV-450, we use the result from [43]: $S_{8}=0.737_{-0.036}^{+0.040}$. For HSC, we use the result from [44]: $S_{8}=0.780_{-0.033}^{+0.030}$.

\section{Supernovae}

The Pantheon dataset of 1048 SNe Ia in the redshift range $0.01<z<2.3$ 83, which provide accurate relative luminosity distances. We note that, as of writing, there is a discrepancy in the Pantheon likelihood implemented in Cobaya and that implemented in Monte Python, another popular cosmological MCMC sampling code. ${ }^{7}$ The origin of this discrepancy is presently unknown, but the $\chi^{2}$ values computed for Pantheon using Cobaya match those found in the official Planck 2018 MCMC chains. ${ }^{8}$ We thus utilize the likelihood as implemented in Cobaya.

\footnotetext{
7 https://github.com/brinckmann/montepython_public/ issues/131

-https://wiki.cosmos.esa.int/planck-legacy-archive/ index.php/Cosmological_Parameters
}

\section{Local Distance Ladder}

The most recent SH0ES measurement $H_{0}=74.03 \pm$ $1.42 \mathrm{~km} / \mathrm{s} / \mathrm{Mpc}$ [15]. We correct a minor bug in the implementation of this likelihood in Cobaya that was present at the time of writing, ${ }^{9}$ which produces a constant offset in the $\chi^{2}$ values. This does not affect parameter constraints (as a constant offset in $\chi^{2}$ is irrelevant in MCMC analyses), but affects the best-fit $\chi^{2}$ values quoted. We have checked that after fixing this bug, the $\chi^{2}$ values produced by the likelihood match those in the official Planck 2018 MCMC chains.

\section{CONSTRAINTS ON THE EDE SCENARIO}

We focus exclusively on the $n=3$ EDE model (see Eq. (11)). This is the best-fit integer-valued $n$ reported in previous analyses [26]. We do not consider non-integer values of $n$, for the reasons discussed below Eq. (5). We note that $n$ is not tightly constrained when allowed to vary [26]. We adopt uniform priors on the effective EDE parameters $f_{\mathrm{EDE}}=[0.001,0.5]$ and $\log _{10}\left(z_{c}\right)=[3.1,4.3]$, and a uniform prior on the initial field displacement $\theta_{i}=[0.1,3.1]$. We examine the prior-dependence of these

\footnotetext{
9 https://github.com/CobayaSampler/cobaya/issues/105
} 
Constraints from Planck 2018 data only: TT+TE+EE

\begin{tabular}{|l|c|c|}
\hline \hline Parameter & $\Lambda$ CDM & EDE $(n=3)$ \\
\hline \hline $\boldsymbol{l n}\left(\mathbf{1 0}^{\mathbf{1 0}} \boldsymbol{A}_{\mathbf{s}}\right)$ & $3.044(3.055) \pm 0.016$ & $3.051(3.056) \pm 0.017$ \\
$\boldsymbol{n}_{\mathbf{s}}$ & $0.9645(0.9659) \pm 0.0043$ & $0.9702(0.9769)_{-0.0069}^{+0.0071}$ \\
$\mathbf{1 0 0 0}_{\mathbf{s}}$ & $1.04185(1.04200) \pm 0.00029$ & $1.04164(1.04168) \pm 0.00034$ \\
$\boldsymbol{\Omega}_{\mathbf{b}} \boldsymbol{h}^{\mathbf{2}}$ & $0.02235(0.02244) \pm 0.00015$ & $0.02250(0.02250) \pm 0.00020$ \\
$\boldsymbol{\Omega}_{\mathbf{c}} \boldsymbol{h}^{\mathbf{2}}$ & $0.1202(0.1201) \pm 0.0013$ & $0.1234(0.1268)_{-0.0030}^{+0.0031}$ \\
$\boldsymbol{\tau}_{\mathbf{r e i o}}(0.0541(0.0587) \pm 0.0076$ & $0.0549(0.0539) \pm 0.0078$ \\
$\log _{\mathbf{1 0}}\left(\boldsymbol{z}_{\boldsymbol{c}}\right)$ & - & $3.66(3.75)_{-0.24}^{+0.28}$ \\
$\boldsymbol{f}_{\mathbf{E D E}}$ & - & $<0.087(0.068)$ \\
$\boldsymbol{\theta}_{\boldsymbol{i}}$ & - & $>0.36(2.96)$ \\
\hline$H_{0}[\mathrm{~km} / \mathrm{s} / \mathrm{Mpc}]$ & $67.29(67.44) \pm 0.59$ & $68.29(69.13)_{-1.00}^{+1.02}$ \\
$\Omega_{\mathrm{m}}$ & $0.3162(0.3147) \pm 0.0083$ & $0.3145(0.3138) \pm 0.0086$ \\
$\sigma_{8}$ & $0.8114(0.8156) \pm 0.0073$ & $0.8198(0.8280)_{-0.0107}^{+0.0109}$ \\
$S_{8}$ & $0.8331(0.8355) \pm 0.0159$ & $0.8393(0.8468) \pm 0.0173$ \\
$\log _{10}(\mathrm{f} / \mathrm{eV})$ & - & $26.57(26.36)_{-0.36}^{+0.39}$ \\
$\log _{10}(\mathrm{~m} / \mathrm{eV})$ & - & $-26.94(-26.90)_{-0.53}^{+0.58}$ \\
\hline
\end{tabular}

TABLE II: The mean (best-fit) $\pm 1 \sigma$ constraints on the cosmological parameters in $\Lambda$ CDM and in the EDE scenario with $n=3$, as inferred from Planck 2018 primary CMB data only (TT+TE+EE). Upper and lower limits are quoted at 95\% CL. Although there is a small contribution to the constraining power in these data from acoustic-peak-smearing due to gravitational lensing, the constraints are dominated by information content from the recombination epoch. The EDE component is not detected here; a two-tailed limit yields $f_{\mathrm{EDE}}=0.033_{-0.026}^{+0.027}$ at $68 \% \mathrm{CL}$, i.e., consistent with zero.

results in Sec. VII in particular by investigating results with uniform priors placed on the particle physics parameters $f$ and $\log _{10}(m)$.

As a benchmark comparison, we also fit the $\Lambda \mathrm{CDM}$ model to the above data sets. We adopt broad uniform priors on the six standard $\Lambda$ CDM parameters (in both the $\Lambda \mathrm{CDM}$ runs and the EDE runs, which of course include these parameters as well). Following the Planck convention, we hold the sum of the neutrino masses fixed to $0.06 \mathrm{eV}$, assuming one massive eigenstate and two massless eigenstates, and fix the effective number of relativistic species $N_{\text {eff }}=3.046$. We also sample and marginalize over the nuisance parameters for all likelihoods using standard methodology. We analyze the MCMC chains using GetDist [37. ${ }^{10}$ All of our chains are publicly available for download. ${ }^{11}$ We encourage interested readers to analyze these data, as the full chains are more informative than any individual summary statistic.

A final comment is in order regarding our parameter constraints. There are two common approaches to obtain marginalized parameter confidence intervals in the case of two-tailed limits. Considering $68 \%$ intervals as an example case, the first approach is to compute a limit such that $32 \%$ of the samples are outside the limit range (symmetrically in the tails), i.e., such that either tail of the marginalized posterior distribution contains $16 \%$ of the samples beyond the quoted limit values. The second approach is to compute an interval between two points with highest equal marginalized probability density (the

10 https://github.com/cmbant/getdist

11 https://users.flatironinstitute.org/ chill/H20_data/ "credible interval") such that the enclosed region contains $68 \%$ of the samples. The two approaches yield identical confidence intervals for Gaussian posterior distributions, but can non-negligibly differ if the distribution is skewed, which is indeed true for the EDE model parameter posterior distributions. Given this choice, we adopt the first approach in the main body of this paper and present estimates obtained with the second method in Appendix E. For either approach, the limits will be quoted as

$$
\text { mean }_{-(\text {mean - lower } 68 \% \text { limit })}^{+(\text {upper } 68 \% \text { limit - mean })}
$$

where "mean" refers to the mean of the marginalized posterior distribution for that parameter.

\section{A. Constraints on EDE from the Primary CMB Alone}

We first consider the Planck 2018 primary CMB TT, $\mathrm{TE}$, and EE power spectrum data. While there is a small contribution to the constraining power from acousticpeak-smearing due to gravitational lensing, the overall constraints are dominated by information from the recombination epoch. This analysis thus examines potential evidence for EDE from early-universe data considered on their own.

We find no evidence for EDE in the Planck 2018 primary CMB data alone. Indeed, the data are powerful enough to set meaningful constraints on the possible existence of an EDE component. The results are tabulated in Tables II. III, and XI and in Figs. 1 and 9 . We find an upper bound $f_{\mathrm{EDE}}<0.087$ at $95 \% \mathrm{CL}$; a two-tailed 
$\chi^{2}$ statistics from Planck 2018 data only: TT+TE+EE

\begin{tabular}{|l|c|c|}
\hline \hline Datasets & $\Lambda$ CDM & EDE \\
\hline Planck 2018 low- $\ell$ TT & 23.4 & 22.1 \\
Planck 2018 low- $\ell$ EE & 397.2 & 396.0 \\
Planck 2018 high- $\ell$ TT+TE+EE & 2344.9 & 2343.3 \\
\hline Total $\chi^{2}$ & 2765.5 & 2761.4 \\
$\Delta \chi^{2}$ & & -4.1 \\
\hline
\end{tabular}

TABLE III: $\chi^{2}$ values for the best-fit $\Lambda$ CDM and EDE models to the primary CMB alone. The reduction in $\chi^{2}$ is 4.1 for the three-parameter EDE extension of $\Lambda \mathrm{CDM}$.

limit yields $f_{\mathrm{EDE}}=0.033_{-0.026}^{+0.027}$ at $68 \%$ CL, i.e., consistent with zero. The initial EDE field displacement $\theta_{i}$ is poorly constrained, and we find a lower bound $\theta_{i}>0.36$ at $95 \% \mathrm{CL}$, a reflection of the fact that at small $f_{\mathrm{EDE}}$ the model is indistinguishable from $\Lambda \mathrm{CDM}$. The timing of EDE is constrained to $\log _{10} z_{c}=3.66_{-0.24}^{+0.28}$, the only indication of a slight CMB preference for EDE. However, the posterior distribution shows significant support on the boundaries of the prior, indicating this result should not be considered to be physically meaningful.

A comparison of the posterior distributions in EDE and $\Lambda \mathrm{CDM}$ can be seen in Fig. 9. We find the Hubble constant in EDE to be $H_{0}=68.29_{-1.00}^{+1.02} \mathrm{~km} / \mathrm{s} / \mathrm{Mpc}$, shifted slightly upwards relative to $\Lambda \mathrm{CDM}$ fit to the same data set, $H_{0}=67.29 \pm 0.59 \mathrm{~km} / \mathrm{s} / \mathrm{Mpc}$, and with a considerably larger error bar. This behaviour (slight upward shift, posterior broadened and skewed towards larger values) is mirrored in $\Omega_{b} h^{2}, \Omega_{c} h^{2}$, and $n_{s}$. We find $S_{8}=0.8393 \pm 0.0173$ in the EDE scenario, larger than the $\Lambda \mathrm{CDM}$ value $S_{8}=0.8331 \pm 0.0159$, both larger than the DES, HSC, and KV-450 constraints, but more so in the EDE case. We also note a considerable degeneracy between $f_{\mathrm{EDE}}$ and $H_{0}$, as well as between $f_{\mathrm{EDE}}$ and $\sigma_{8}$ (see Fig. 1).

The goodness-of-fit to the primary CMB anisotropies, as quantified by the $\chi^{2}$-statistic, is only marginally improved in the EDE three-parameter extension of $\Lambda \mathrm{CDM}$. The $\chi^{2}$ statistics for each primary CMB likelihood are given in Table III. We find an improvement $\Delta \chi^{2}=-4.1$, with nearly equal contributions from the low- $\ell$ TT, low- $\ell$ $\mathrm{EE}$, and high- $\ell \mathrm{TT}+\mathrm{TE}+\mathrm{EE}$ likelihoods. With three additional parameters, this is not a significant improvement over $\Lambda$ CDM.

\section{B. Constraints from Primary CMB, CMB Lensing, BAO, RSD, SNIa, and SHOES Data}

We now supplement the Planck 2018 primary CMB anisotropy data with Planck 2018 CMB lensing, BAO, RSD, supernova, and local distance ladder data. This combination of data sets, with the exception of our use of Planck 2018 data in place of 2015 data, was the basis for the conclusions in 25] and [26]. The posterior distributions are shown in Figs. 1 and 10 the best-fit parameters and constraints are tabulated in Tables IV and XII, and the $\chi^{2}$ values are given in Table $\mathrm{V}$.

We find $f_{\mathrm{EDE}}=0.098 \pm 0.032$, i.e., a $3.1 \sigma$ detection of EDE, when using this combination of data sets. This value is larger than the 95\% CL upper limit from the CMB alone, as can be appreciated from Fig. 1 indicating minor tension between the data sets in the context of EDE. The shift in $f_{\mathrm{EDE}}$ is driven by the $H_{0}$ tension combined with the $f_{\mathrm{EDE}}-H_{0}$ degeneracy of the EDE fit to the primary $\mathrm{CMB}$, which allows larger $H_{0}$ values without substantially degrading the fit to the latter. These results are consistent with those presented in previous work 25, 26. While the RSD and CMB lensing likelihoods provide some LSS information in this data set combination, their error bars are large enough so as to not strongly disfavor the region of parameter space that can resolve the Hubble tension. The somewhat higher value of $\sigma_{8}$ found in the Planck CMB lensing analysis [77 in comparison to the DES, HSC, and KV-450 galaxy weak lensing analyses also plays an important role here.

We find $H_{0}=70.98 \pm 1.05 \mathrm{~km} / \mathrm{s} / \mathrm{Mpc}$, reducing the tension with SH0ES to $\approx 1.7 \sigma$, in comparison with the $\Lambda \mathrm{CDM}$ value $H_{0}=68.17 \pm 0.39 \mathrm{~km} / \mathrm{s} / \mathrm{Mpc}$, the latter in $4.0 \sigma$ tension with SHOES. The EDE reduction in the tension is consistent with the conclusions of past works. However we note the relative decrease in tension is in part driven by a tripling of the error bar in the broadened parameter space.

As anticipated, the tension with low-redshift LSS $S_{8}$ constraints is worsened in the EDE fit, as compared to $\Lambda \mathrm{CDM}$. We find $\sigma_{8}=0.8337 \pm 0.0105$ for EDE, $\sigma_{8}=$ $0.8086 \pm 0.0060$ for $\Lambda \mathrm{CDM}$, and $\Omega_{\mathrm{m}}=0.3025 \pm 0.0051$ and $\Omega_{\mathrm{m}}=0.3044 \pm 0.0051$ for EDE and $\Lambda \mathrm{CDM}$, respectively. This drives an enhanced $S_{8}$ tension in EDE; $S_{8}=0.8372 \pm 0.0127$, in moderate $2.2 \sigma$ tension with DES-Y1, for example. In contrast, for $\Lambda \mathrm{CDM}$ fit to this combination of data sets, we find $S_{8}=0.8145 \pm 0.0099$, which differs from DES at $1.5 \sigma$, i.e., the two are statistically consistent.

The impact of EDE on the fit to the other $\Lambda \mathrm{CDM}$ parameters is similar to that observed in the fit to the primary CMB alone. Relative to the $\Lambda \mathrm{CDM}$ fit to the same data sets, we find a shift upwards of $\Omega_{c} h^{2}, \Omega_{b} h^{2}$, $n_{s}$, and a significant broadening of the posteriors. The shift is most noticeable in $\Omega_{c} h^{2}$, which is the driver of the changes to the matter power spectrum $P(k)$ observed in Sec. IV. We find $\Omega_{\mathrm{c}} h^{2}=0.12899 \pm 0.00390$ in EDE and $\Omega_{\mathrm{c}} h^{2}=0.11830 \pm 0.00085$ in $\Lambda \mathrm{CDM}$.

The EDE parameters $z_{c}$ and $\theta_{i}$ are well constrained in comparison to the fit to the primary $\mathrm{CMB}$ alone (see Fig. 1). The EDE critical redshift $z_{c}$ is found to be $\log _{10}\left(z_{c}\right)=3.63_{-0.10}^{+0.17}$. The posterior exhibits a weakly bimodal distribution, mirroring the results of [26, with a tail towards large $\log _{10}\left(z_{c}\right)$. As discussed in [26], the subdominant peak is driven by high- $\ell$ polarization data; it does not have an obvious physical interpretation and could simply be due to a noise fluctuation. On the other hand, the tail correlates with large $f_{\mathrm{EDE}}$ and small $\Omega_{c} h^{2}$; this is simply the region of parameter space where the 


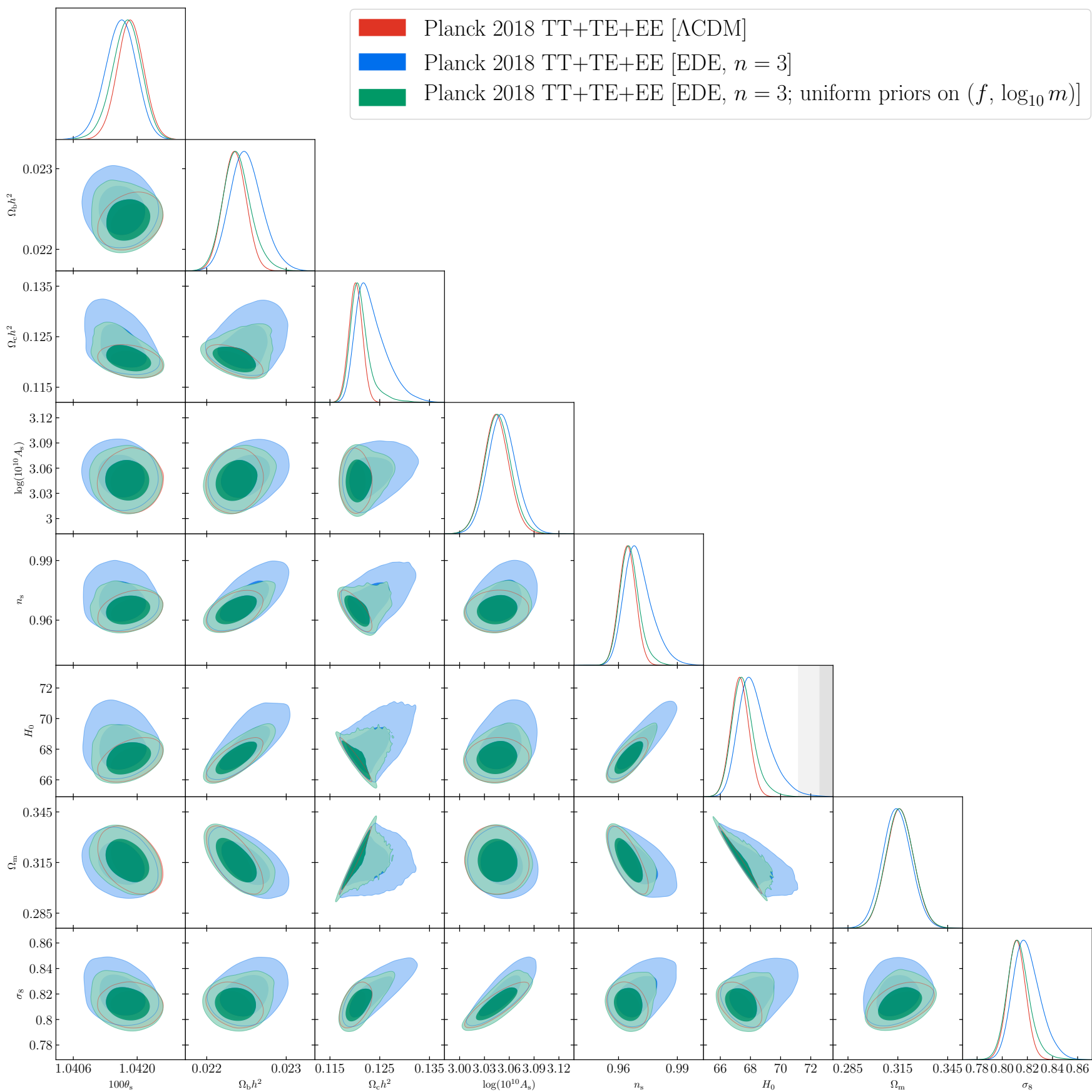

FIG. 9: Cosmological parameter constraints from the Planck 2018 primary CMB data alone (TT+TE+EE). The red (blue) contours show $1 \sigma$ and $2 \sigma$ posteriors in the $\Lambda$ CDM (EDE, $n=3$ ) models. We do not plot $\tau$, as it is unchanged in the EDE fit. The most significant changes in the EDE fit (compared to $\Lambda$ CDM) are increases in $\Omega_{c} h^{2}, n_{s}, H_{0}$, and $\sigma_{8}$, as well as broadening of the error bars on these parameters. However, the change in $H_{0}$ is not large enough to reconcile the tension with the SH0ES constraint (shown in the gray bands in the $H_{0}$ panel). The green contours show posteriors for the EDE model, but with uniform priors placed on the (physical) particle physics parameters $f$ and $\log _{10}(m)$, rather than on the effective EDE parameters $f_{\mathrm{EDE}}$ and $\log _{10}\left(z_{c}\right)$. Comparison of the blue and green contours indicates that the physical priors strongly downweight EDE models than can resolve the $H_{0}$ tension; see Sec. VII for further discussion. 


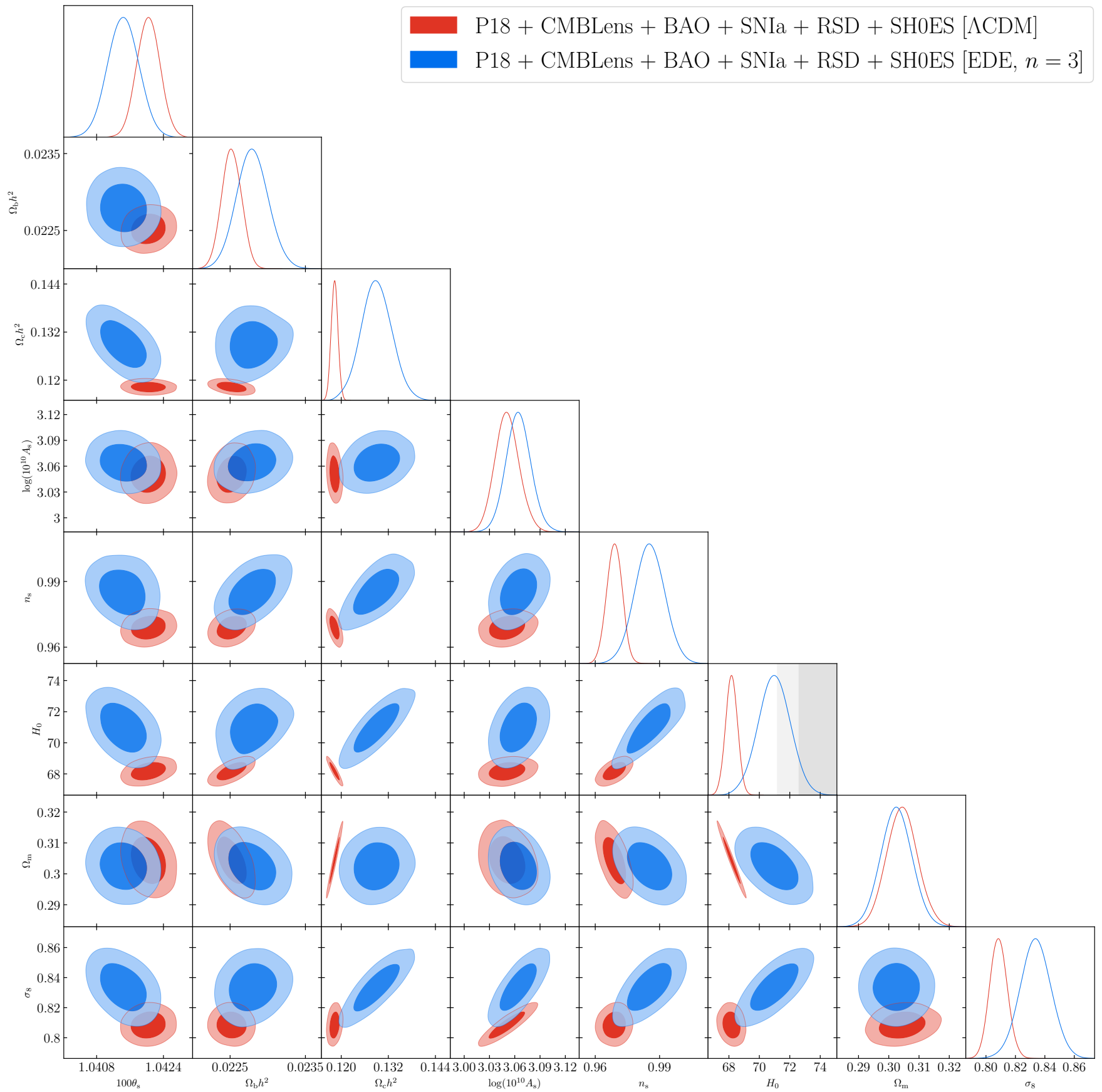

FIG. 10: Cosmological parameter constraints from the combination of Planck 2018 primary CMB data (TT+TE+EE); Planck 2018 CMB lensing data; BAO data from 6dF, SDSS DR7, and SDSS DR12; Pantheon SNIa data; the latest SH0ES $H_{0}$ constraint; and SDSS DR12 RSD data. This data set combination matches that used in [26], with the exception of Planck 2018 data used here in place of 2015 data. The red (blue) contours show $1 \sigma$ and $2 \sigma$ posteriors in the $\Lambda$ CDM (EDE, $n=3$ ) models. We do not plot $\tau$, as it is essentially unchanged in the EDE fit. Many parameters shift by a non-negligible amount in the EDE fit (compared to $\Lambda \mathrm{CDM}$ ), including increases in $\Omega_{b} h^{2}, \Omega_{c} h^{2}, n_{s}, H_{0}$, and $\sigma_{8}$ and a decrease in $100 \theta_{s}$, as well as broadening of the error bars on these parameters. The increase in $H_{0}$ is large enough to mostly reconcile the tension with the SH0ES-only constraint (shown in the gray bands). The EDE component is detected at $3.1 \sigma$ significance using this combination of data sets, consistent with earlier work [25, 26]. 
Constraints from Planck 2018 TT+TE+EE + CMB Lensing, BAO, SNIa, SH0ES, and RSD

\begin{tabular}{|l|c|c|}
\hline \hline Parameter & $\Lambda$ CDM & EDE $(n=3)$ \\
\hline \hline $\boldsymbol{\operatorname { l n }}\left(\mathbf{1 0}^{\mathbf{1 0}} \boldsymbol{A}_{\mathbf{s}}\right)$ & $3.051(3.047) \pm 0.014$ & $3.064(3.058) \pm 0.015$ \\
$\boldsymbol{n}_{\mathbf{s}}$ & $0.9689(0.9686) \pm 0.0036$ & $0.9854(0.9847)_{-0.69}^{+0.70}$ \\
$\mathbf{1 0 0}_{\mathbf{s}}$ & $1.04204(1.04209) \pm 0.00028$ & $1.04144(1.04119) \pm 0.00037$ \\
$\boldsymbol{\Omega}_{\mathbf{b}} \boldsymbol{h}^{\mathbf{2}}$ & $0.02252(0.02249) \pm 0.00013$ & $0.02280(0.02286) \pm 0.00021$ \\
$\boldsymbol{\Omega}_{\mathrm{c}} \boldsymbol{h}^{\mathbf{2}}$ & $0.11830(0.11855) \pm 0.00085$ & $0.12899(0.12999) \pm 0.00390$ \\
$\boldsymbol{\tau}_{\mathbf{r e i o}}$ & $0.0590(0.0566) \pm 0.0072$ & $0.0573(0.0511) \pm 0.0071$ \\
$\log _{\mathbf{1 0}}\left(\boldsymbol{z}_{\boldsymbol{c}}\right)$ & - & $3.63(3.59)_{-0.10}^{+0.17}$ \\
$\boldsymbol{f}_{\mathbf{E D E}}$ & - & $0.098(0.105) \pm 0.032$ \\
$\boldsymbol{\theta}_{\boldsymbol{i}}$ & - & $2.58(2.71)_{-0.09}^{+0.29}$ \\
\hline$H_{0}[\mathrm{~km} / \mathrm{s} / \mathrm{Mpc}]$ & $68.17(68.07) \pm 0.39$ & $70.98(71.15) \pm 1.05$ \\
$\Omega_{\mathrm{m}}$ & $0.3044(0.3058) \pm 0.0051$ & $0.3025(0.3032) \pm 0.0051$ \\
$\sigma_{8}$ & $0.8086(0.8081) \pm 0.0060$ & $0.8337(0.8322) \pm 0.0105$ \\
$S_{8}$ & $0.8145(0.8158) \pm 0.0099$ & $0.8372(0.8366) \pm 0.0127$ \\
$\log _{10}(f / \mathrm{eV})$ & - & $26.64(26.63)_{-0.15}^{+0.08}$ \\
$\log _{10}(\mathrm{~m} / \mathrm{eV})$ & - & $-27.15(-27.27)_{-0.22}^{+0.34}$ \\
\hline
\end{tabular}

TABLE IV: The mean (best-fit) $\pm 1 \sigma$ constraints on the cosmological parameters in $\Lambda$ CDM and in the EDE scenario with $n=3$, as inferred from the combination of Planck 2018 primary CMB data (TT+TE+EE); Planck 2018 CMB lensing data; BAO data from 6dF, SDSS DR7, and SDSS DR12; Pantheon SNIa data; the latest SH0ES $H_{0}$ constraint; and SDSS DR12 RSD data. The EDE component is detected at $3.1 \sigma$ significance.

$\chi^{2}$ statistics from the fit to Planck 2018 TT+TE+EE +
\begin{tabular}{|l|c|c|} 
CMB Lensing, BAO, SNIa, SH0ES, and RSD \\
\hline \hline Datasets & $\Lambda$ CDM & EDE \\
\hline \hline CMB TT, EE, TE: & & \\
Planck 2018 low- $\ell$ TT & 22.8 & 21.4 \\
Planck 2018 low- $\ell$ EE & 396.4 & 395.8 \\
Planck 2018 high- $\ell$ & 2346.8 & 2346.9 \\
TT+TE+EE & & \\
LSS: & 8.9 & 9.5 \\
Planck CMB lensing & 0.0015 & 0.000002 \\
BAO (6dF) & 1.6 & 1.7 \\
BAO (DR7 MGS) & 5.9 & 6.5 \\
BAO+RSD (DR12 BOSS) & & \\
Supernovae: & 1034.8 & 1034.8 \\
Pantheon & 17.6 & 4.1 \\
SH0ES & 3834.8 & 3820.7 \\
\hline Total $\chi^{2}$ & & -14.1 \\
$\Delta \chi^{2}$
\end{tabular}

TABLE V: $\chi^{2}$ values for the best-fit $\Lambda$ CDM and EDE models to $\mathrm{CMB}+\mathrm{CMB}$ Lensing $+\mathrm{BAO}+\mathrm{SNIa}+\mathrm{SHOES}+\mathrm{RSD}$ data. The reduction in $\chi^{2}$ is 14.1 for the three-parameter EDE extension of $\Lambda \mathrm{CDM}$, driven almost entirely by the improved fit to SH0ES. However, it is also notable that the fit to the CMB is not degraded, even as the fit to SHOES improves.

sound horizon and subsequent cosmology is left unaffected by the EDE, even for fairly large values of $f_{\mathrm{EDE}}$. We find a strong preference for a large initial field displacement, $\theta_{i}=2.58_{-0.09}^{+0.29}$, consistent with the finding of [26] that the best-fit models lie outside the regime wherein the scalar field potential can be expanded as a monomial.

The $\chi^{2}$ statistics for each likelihood in this fit are given in Table $\mathrm{V}$. The $3.1 \sigma$ detection of EDE is accompanied by a marked increase in the goodness-of-fit as compared to
$\Lambda \mathrm{CDM}$. The EDE improvement in the total $\chi^{2}$-statistic is $\Delta \chi^{2}=-14.1$, driven almost entirely by the improved fit to SH0ES, $\Delta \chi_{\mathrm{SHOES}}^{2}=-13.5$, counteracted by a slightly worsened fit to the LSS data, $\Delta \chi_{\mathrm{LSS}}^{2}=+1.3$ in total. The latter hints at the potential for additional LSS likelihoods to substantially constrain EDE, particularly via the sensitivity to $P(k)$ as motivated by Fig. 8 .

\section{Including the (Early) Dark Energy Survey}

We now expand our analysis to include likelihoods from the DES-Y1 data set, in particular the "3x2pt" likelihood comprised of photometric galaxy clustering, galaxy-galaxy lensing, and cosmic shear two-point correlation functions [38. We jointly analyze all likelihoods described in the previous two subsections and the DESY1 3x2pt likelihood.

As mentioned earlier, we use the "Halofit" prescription 66, 67] to compute the non-linear matter power spectrum, following the DES methodology 38. Thus we assume that the "Halofit" fitting function calibration remains valid in the EDE models under consideration. To justify this assumption, we note that in the models capable of addressing the $H_{0}$ tension and fitting the CMB data, the deviation from a $\Lambda \mathrm{CDM} P(k)$ is not particularly large, since $f_{\mathrm{EDE}} \lesssim 0.1$. The test that we perform in Appendix B (see Figs. 17, 18, and 19, which are described near the beginning of the next subsection) provides a further justification for the validity of using Halofit. There, we compare results obtained when using the full DES-Y1 3x2pt likelihood, which relies on the Halofit non-linear $P(k)$, to results obtained when imposing a Gaussian prior on $S_{8}$ corresponding to the DES-Y1 result, which only requires linear theory to compute. If 
Constraints from Planck 2018 TT+TE+EE + CMB Lensing, BAO, SNIa, SH0ES, RSD, and DES-Y1

\begin{tabular}{|l|c|c|}
\hline \hline Parameter & $\Lambda$ CDM & EDE $(n=3)$ \\
\hline \hline $\boldsymbol{l n}\left(\mathbf{1 0}^{\mathbf{1 0}} \boldsymbol{A}_{\mathbf{s}}\right)$ & $3.049(3.049) \pm 0.014$ & $3.058(3.064) \pm 0.015$ \\
$\boldsymbol{n}_{\mathbf{s}}$ & $0.9704(0.9698) \pm 0.0035$ & $0.9838(0.9909)_{-0.0075}^{+0.0074}$ \\
$\mathbf{1 0 0 0}_{\mathbf{s}}$ & $1.04208(1.04183) \pm 0.00028$ & $1.04162(1.04172) \pm 0.00036$ \\
$\boldsymbol{\Omega}_{\mathbf{b}} \boldsymbol{h}^{\mathbf{2}}$ & $0.02258(0.02260) \pm 0.00013$ & $0.02285(0.02304) \pm 0.00021$ \\
$\boldsymbol{\Omega}_{\mathbf{c}} \boldsymbol{h}^{\mathbf{2}}$ & $0.11752(0.11810) \pm 0.00078$ & $0.1251(0.1254)_{-0.0037}^{+0.0035}$ \\
$\boldsymbol{\tau}_{\mathbf{r e i o}}$ & $0.0590(0.0584) \pm 0.0072$ & $0.0581(0.0626) \pm 0.0072$ \\
$\log _{\mathbf{1 0}}\left(\boldsymbol{z}_{\boldsymbol{c}}\right)$ & - & $3.69(3.84)_{-0.15}^{+0.18}$ \\
$\boldsymbol{f}_{\mathbf{E D E}}$ & - & $0.077(0.088)_{-0.034}^{+0.032}$ \\
$\boldsymbol{\theta}_{\boldsymbol{i}}$ & - & $2.58(2.89)_{-0.15}^{+0.32}$ \\
\hline$H_{0}[\mathrm{~km} / \mathrm{s} / \mathrm{Mpc}]$ & $68.52(68.24) \pm 0.36$ & $70.75(71.05)_{-1.09}^{+1.05}$ \\
$\Omega_{\mathrm{m}}$ & $0.2998(0.3035) \pm 0.0046$ & $0.2970(0.2954) \pm 0.0047$ \\
$\sigma_{8}$ & $0.8054(0.8067) \pm 0.0057$ & $0.8228(0.8263)_{-0.0101}^{+0.0099}$ \\
$S_{8}$ & $0.8051(0.8115) \pm 0.0087$ & $0.8186(0.8199) \pm 0.0109$ \\
$\log _{10}(\mathrm{f} / \mathrm{eV})$ & - & $26.57(26.47)_{-0.16}^{+0.11}$ \\
$\log _{10}(\mathrm{~m} / \mathrm{eV})$ & - & $-27.03(-26.76)_{-0.32}^{+0.33}$ \\
\hline
\end{tabular}

TABLE VI: The mean (best-fit) $\pm 1 \sigma$ constraints on the cosmological parameters in $\Lambda$ CDM and in the EDE scenario with $n=3$, as inferred from the combination of Planck 2018 primary CMB data (TT+TE+EE); Planck 2018 CMB lensing data; BAO data from $6 \mathrm{dF}$, SDSS DR7, and SDSS DR12; Pantheon SNIa data; the latest SH0ES $H_{0}$ constraint; SDSS DR12 RSD data; and DES-Y1 3x2pt data. With the inclusion of the DES data, the evidence for the EDE component decreases to $2.3 \sigma$. A one-sided upper limit on the EDE fraction yields $f_{\mathrm{EDE}}<0.127$ at $95 \% \mathrm{CL}$. The non-negligible discrepancy between the best-fit and mean posterior values is a reflection of the underlying tension between these data sets (i.e., SH0ES and all other data sets) in the context of this model.

the Halofit prediction of the non-linear $P(k)$ were highly inaccurate in the EDE models under consideration, then the posteriors obtained for the EDE parameters would a priori be very different in the two approaches. The test thus not only verifies that the information content in the DES-Y1 data is almost entirely contained in the $S_{8}$ result, as discussed further in the next subsection, but also verifies that the non-linear modeling used in the $3 \mathrm{x} 2 \mathrm{pt}$ likelihood is sufficiently accurate, even for the EDE models.

The posterior distributions for our analysis including the full DES-Y1 3x2pt likelihood are shown in Figs. 1 and 11, the parameter constraints are tabulated in Tables $\mathrm{VI}$ and XIII, and the $\chi^{2}$ values are given in Table VII. With the inclusion of the DES-Y1 3x2pt likelihood, the evidence for EDE decreases to $2.3 \sigma$. We find $f_{\mathrm{EDE}}=0.077_{-0.034}^{+0.032}$, shifted downwards from the result without DES (Sec. VIB) to come into statistical agreement with the upper bound from the primary CMB anisotropies (Sec. VIA). A one-sided upper limit yields $f_{\mathrm{EDE}}<0.127$ at $95 \% \mathrm{CL}$. The initial field displacement and critical redshift are constrained to be $\theta_{i}=2.58_{-0.15}^{+0.32}$, and $\log _{10}\left(z_{c}\right)=3.69_{-0.15}^{+0.18}$. Broadly speaking, the EDE parameter posteriors move towards the CMB-only results, as can be appreciated from Fig. 1. It is notable that the best-fit parameter values in Table VI differ by a non-negligible amount from the mean of the posteriors. This reflects the fact that the data sets are pulling the model parameters in opposite directions: the SH0ES data can only be fit by increasing $f_{\mathrm{EDE}}$ (and thus moving other parameters along their degeneracies with it), but the CMB and LSS data do not prefer large $f_{\mathrm{EDE}}$. In a proper Bayesian sense, it is likely that the data sets are in tension and should not be combined in the first place (e.g., [84 86]).

The downward shift in $f_{\mathrm{EDE}}$ when DES-Y1 3x2pt data are added to the combined data set of Sec. VIB can be understood in terms of the interplay between $\sigma_{8}, \Omega_{m}$, $H_{0}$, and $f_{\mathrm{EDE}}$. As discussed in 38 in the context of $\Lambda \mathrm{CDM}$, the precise DES measurement of $\Omega_{m}$ breaks the $\Omega_{m}-H_{0}$ degeneracy in the $\Lambda \mathrm{CDM}$ fit to the CMB, shifting $H_{0}$ to larger values. In the EDE scenario, the impact of DES measurements on $H_{0}$ is the reverse, caused by a marked correlation between $\sigma_{8}$ and $H_{0}$, which can be observed in both Fig. 9 and Fig. 10. This is manifested in the discrepancy between the DES matter power spectrum constraints and the predictions of the EDE model fit to the data sets in Fig. 10 (see Fig. 8). As a result, the DES likelihood drives $\overline{H_{0}}$ to lower values. The tight correlation between $H_{0}$ and $f_{\mathrm{EDE}}$ then leads to a smaller value for $f_{\mathrm{EDE}}$.

This is borne out in the $H_{0}$ constraints. We find $H_{0}=70.75_{-1.09}^{+1.05} \mathrm{~km} / \mathrm{s} / \mathrm{Mpc}$, in mild $1.9 \sigma$ tension with SH0ES. This is shifted slightly downwards from the value in the fit without DES, $H_{0}=70.98 \pm 1.05 \mathrm{~km} / \mathrm{s} / \mathrm{Mpc}$. In contrast, the $\Lambda \mathrm{CDM}$ constraint is raised to $H_{0}=$ $68.52 \pm 0.36 \mathrm{~km} / \mathrm{s} / \mathrm{Mpc}$, decreasing the $\Lambda \mathrm{CDM}$ tension with SH0ES to $3.8 \sigma$. This contrary motion in $H_{0}$ values is reflected in the SHOES contribution to the $\chi^{2}$-statistic, $\Delta \chi_{\text {SHOES }}^{2}=-12.2$, as seen in Table VII, which is slightly lower than the improvement when DES was not included, $\Delta \chi_{\text {SHOES }}^{2}=-13.5$, as seen in Table $\mathrm{V}$.

It is also notable that the $\sigma_{8}$ posterior matches closely that of the fit to the primary CMB alone, as seen in Fig. 1. erasing the shift observed in the fit without 
$\chi^{2}$ statistics from the fit to Planck $2018 \mathrm{TT}+\mathrm{TE}+\mathrm{EE}+$ CMB Lensing, BAO, SNIa, SH0ES, RSD, and DES-Y1

\begin{tabular}{|l|c|c|}
\hline \hline Datasets & $\Lambda$ CDM & EDE \\
\hline \hline CMB TT, EE, TE: & & \\
Planck 2018 low- $\ell$ TT & 22.7 & 20.5 \\
Planck 2018 low- $\ell$ EE & 396.8 & 397.7 \\
Planck 2018 high- $\ell$ & 2347.6 & 2350.2 \\
TT+TE+EE & & \\
LSS: & 9.0 & 9.6 \\
Planck CMB lensing & 0.0001 & 0.04 \\
BAO (6dF) & 1.8 & 2.4 \\
BAO (DR7 MGS) & 5.9 & 6.9 \\
BAO+RSD (DR12 BOSS) & 507.8 & 508.7 \\
DES-Y1 & & \\
Supernovae: & 1034.8 & 1034.8 \\
Pantheon & 16.6 & 4.4 \\
SH0ES & 4343.0 & 4335.2 \\
Total $\chi^{2}$ & & -7.8 \\
$\Delta \chi^{2}$
\end{tabular}

TABLE VII: The $\chi^{2}$ statistics in the fit to $\mathrm{CMB}+\mathrm{CMB}$ Lensing $+\mathrm{BAO}+\mathrm{SNIa}+\mathrm{SH} 0 \mathrm{ES}+\mathrm{RSD}+\mathrm{DES}$ data for the best-fit $\Lambda \mathrm{CDM}$ and EDE models. The reduction in $\chi^{2}$ is $\Delta \chi^{2}=-7.8$ for the three-parameter EDE extension of $\Lambda$ CDM. As in Table $\mathrm{V}$ this decrease is driven almost entirely by the improved fit to $\operatorname{SHOES}\left(\Delta \chi_{\mathrm{SHOES}}^{2}=-12.2\right)$. The fit to the primary $\mathrm{CMB}$ actually worsens slightly $\left(\Delta \chi_{\mathrm{CMB}}^{2}=1.3\right)$, as does the fit to LSS data $\left(\Delta \chi_{\mathrm{LSS}}^{2}=3.1\right)$.

DES (Sec. VIB). Generated by the degeneracy between $f_{\mathrm{EDE}}$ and $\sigma_{8}$, this is a further indication that both LSS and CMB observations are statistically consistent with $f_{\mathrm{EDE}}=0$. This is matched by shifts in $S_{8}$ and $\Omega_{m}$; we find $S_{8}=0.8186 \pm 0.0109$ and $\Omega_{m}=0.2970 \pm 0.0047$, both in statistical agreement with DES-only measurements.

The $\chi^{2}$-statistic for each likelihood (in the joint-bestfit model) is given in Table VII The EDE improvement in the total $\chi^{2}$-statistic with respect to $\Lambda \mathrm{CDM}$ is $\Delta \chi^{2}=-7.8$, noticeably worse than the improvement in fit with DES excluded (Table V). The $\chi^{2}$ improvement here is again driven almost fully by the improved fit to SH0ES data $\left(\Delta \chi_{\mathrm{SHOES}}^{2}=-12.2\right)$; the fit to the primary $\mathrm{CMB}$ and LSS data actually worsen slightly $\left(\Delta \chi_{\mathrm{CMB}}^{2}=1.3\right.$ and $\left.\Delta \chi_{\mathrm{LSS}}^{2}=3.1\right)$. The EDE model does not appear to provide a region of parameter space that is in concordance with all cosmological data sets.

\section{Additional LSS Data: KiDS+VIKING-450 and Hyper Suprime-Cam}

The KV-450 42, 43. and HSC 44, surveys provide complementary data sets to DES. However, rather than perform the computationally expensive MCMC analysis of directly sampling from these likelihoods in addition to DES-Y1, we opt instead to approximate the KV-450 and HSC data sets by priors on $S_{8}$, corresponding to the constraints $S_{8}=0.737_{-0.036}^{+0.040}$ and $S_{8}=0.780_{-0.033}^{+0.030}$, re- spectively. ${ }^{12}$

To validate this procedure, we first test it with the DES-Y1 3x2pt data, for which we have the full likelihood, as well as the $S_{8}$ constraint given in [38. In Figs. 17 and 18 in Appendix B, we compare the posterior distributions for cosmological parameters in the EDE scenario fit to the combined data set with DES 3x2pt data included (i.e., the results from Sec. VIC) to those with the DES 3x2pt data replaced by a Gaussian prior on $S_{8}$ given by the DES result $S_{8}=0.773_{-0.020}^{+0.026}$, i.e., an $S_{8}$ prior imposed on the results of Sec. VIB. The posterior distributions are in near perfect agreement, for both the EDE parameters (Fig. 17) and standard $\Lambda$ CDM parameters (Fig. 18). Quantitatively, for example, the marginalized constraints on the EDE fraction and critical redshift are $f_{\mathrm{EDE}}=0.076_{-0.033}^{+0.032}$ and $\log _{10}\left(z_{c}\right)=3.66_{-0.14}^{+0.18}$, which are essentially identical to those found in the full analysis in Table VI, $f_{\mathrm{EDE}}=0.077_{-0.034}^{+0.032}$ and $\log _{10}\left(z_{c}\right)=3.69_{-0.15}^{+0.18}$. Quantitative results for the other parameters are of similar fidelity.

As a further test, in Fig. 19 we also perform the same comparison in the $\Lambda \mathrm{CDM}$ model fit to these data sets. We again find excellent agreement in the posteriors, albeit with a small shift visible in $\Omega_{m}$ due to the fact that the DES 3x2pt likelihood does constrain this parameter, but the $S_{8}$ prior approach neglects this information. The correlation of $\Omega_{m}$ with $\Omega_{c} h^{2}$ and $H_{0}$ then produces small $(\ll 1 \sigma)$ shifts in these parameters. Overall, we conclude that the agreement between the $S_{8}$ prior approach and the full DES 3x2pt likelihood is excellent in both the EDE and $\Lambda$ CDM models. Fundamentally, this arises from the fact that DES only weakly constrains the non- $S_{8}$ parameters in comparison to Planck.

Guided by this excellent agreement for DES-Y1, we include HSC and KV-450 constraints by imposing appropriate Gaussian priors on $S_{8}$ using the values given above. We apply this methodology to both the $\Lambda$ CDM and EDE models. The results of this analysis are shown in Figs. 1 and 11, and tabulated in Tables VIII and XIV. We treat the three surveys as independent, which is accurate since the sky overlap between the DES-Y1, KV-450, and HSC-Y1 survey regions is small. There is roughly 20 $\operatorname{deg}^{2}$ overlap between DES-Y1 and HSC-Y1; no overlap between DES-Y1 and KV-450; and roughly $70 \mathrm{deg}^{2}$ overlap between HSC-Y1 and KV-450. Any covariance due to the latter overlap is further weakened by the significantly greater depth of the HSC survey compared to KV-450 (roughly double the effective number of source galaxies), i.e., the redshift window functions of the modes probed by the surveys are quite different. These sky overlap numbers are approximate estimates based on the footprints given in 87, 88, and 89 for DES-Y1, HSC-Y1, and $\mathrm{KV}-450$, respectively. Regarding possible common

\footnotetext{
12 In addition, as of this writing, we are not aware of a publicly available likelihood for the HSC data.
} 


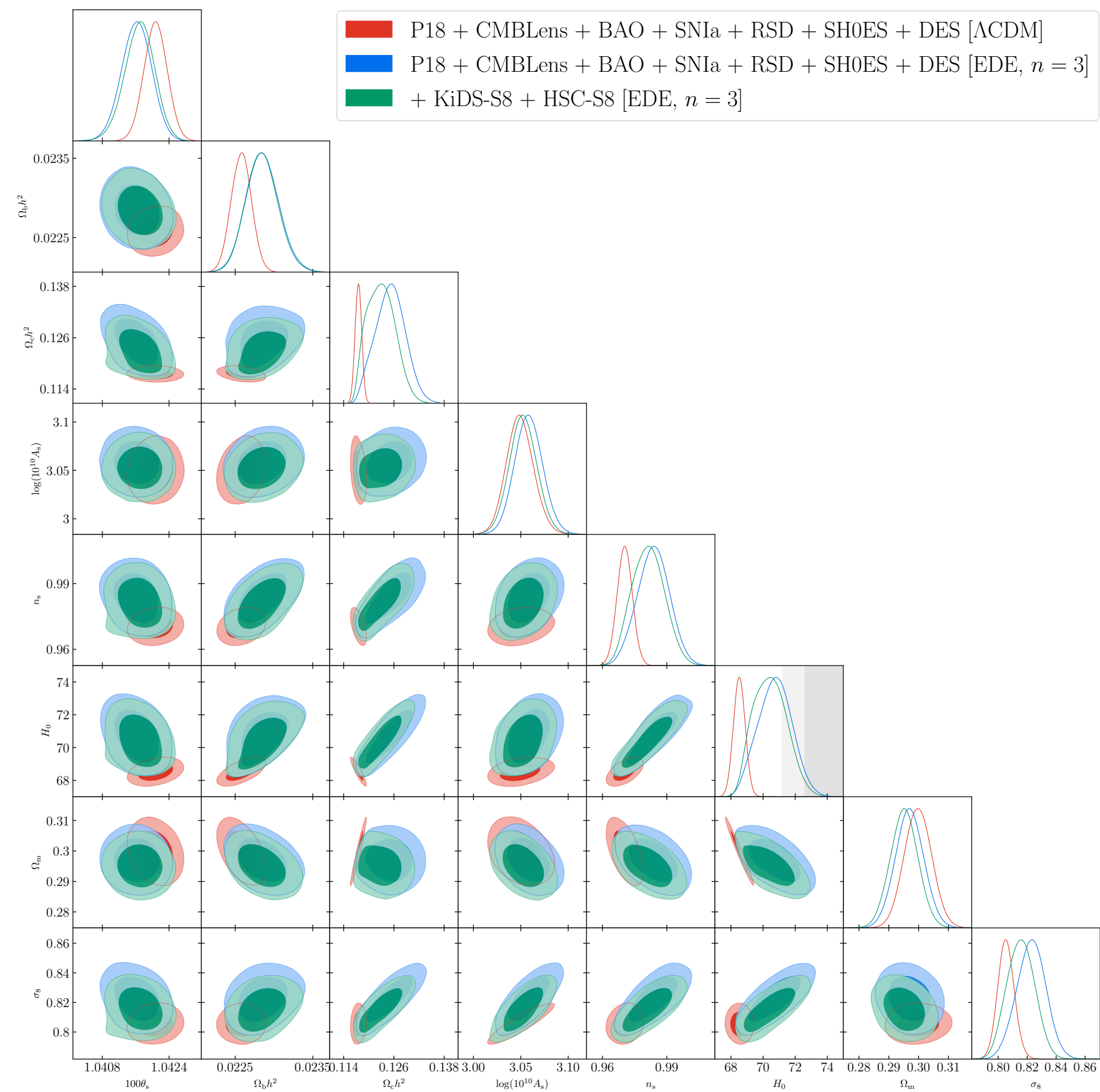

FIG. 11: Cosmological parameter constraints from the combination of Planck 2018 primary CMB data (TT+TE+EE); Planck 2018 CMB lensing data; BAO data from 6dF, SDSS DR7, and SDSS DR12; Pantheon SNIa data; the latest SH0ES $H_{0}$ constraint; SDSS DR12 RSD data; and the DES-Y1 3x2pt data. The red (blue) contours show $1 \sigma$ and $2 \sigma$ posteriors in the $\Lambda \mathrm{CDM}$ (EDE, $n=3$ ) models. We do not plot $\tau$, as it is essentially unchanged in the EDE fit. The green contours show posteriors in the EDE model when further including priors on $S_{8}$ derived from KiDS and HSC data (as an approximation to the use of full likelihoods from these data sets). Inclusion of the DES data weakens the preference for EDE seen in Fig. 10 this shift is due to the discrepancy between the DES matter power spectrum constraints and the predictions of the EDE model fit to the data sets in Fig. 10 (see Fig. 8). Inclusion of the KiDS and HSC $S_{8}$ priors further weakens the evidence for EDE, bringing it well under $2 \sigma$. The upshot is that the increase in $H_{0}$ seen in the EDE fit in Fig. 10 is now reduced, and the increased tension with SHOES alone (shown in the gray bands) is apparent. 
systematics, the surveys also differ in their photo- $z$ calibration: KV-450 uses a combined data set comprised of a large number of spectroscopic surveys 43, while DES-Y1 and HSC-Y1 both use the COSMOS photo- $z$ catalogue 90].

The evidence for EDE is weakened to below $2 \sigma$ by the inclusion of HSC and KV-450 information. We find $f_{\mathrm{EDE}}=0.062_{-0.033}^{+0.032}$, statistically consistent with $f_{\mathrm{EDE}}=$ 0 . The $f_{\text {EDE }}$ posterior exhibits substantial support at the boundary $f_{\mathrm{EDE}}=0$, and we find a one-sided upper limit $f_{\mathrm{EDE}}<0.112$ at $95 \% \mathrm{CL}$. The Hubble constant shifts downward to $H_{0}=70.45_{-1.08}^{+1.05} \mathrm{~km} / \mathrm{s} / \mathrm{Mpc}$, in mild $2 \sigma$ tension with the SH0ES-only constraint.

The EDE posterior distributions for the standard $\Lambda \mathrm{CDM}$ parameters are shown in Fig. 11 (green). The HSC and KV-450 $S_{8}$ priors lead to a substantial shift to smaller values of $\Omega_{c} h^{2}$ and $\sigma_{8}$, while $\Omega_{b} h^{2}$ is unaffected. This is partnered with the shift in $H_{0}$ to reduce $S_{8}$ to $S_{8}=0.8090 \pm 0.0100$, in statistical agreement with the $S_{8}$ measurements of DES, HSC, and KV-450. The EDE parameter posteriors (Fig. 1) move towards those in the fit of EDE to the primary CMB alone, both consistent with $f_{\mathrm{EDE}}=0$.

\section{E. Walking Barefoot: EDE without SHOES}

To complete our analysis of constraints on EDE from these data sets and data set combinations, we consider the cosmological constraints when the SH0ES measurement $H_{0}=74.03 \pm 1.42 \mathrm{~km} / \mathrm{s} / \mathrm{Mpc}$ [15] is excluded from the combined data set. We impose an additional inverseGaussian $H_{0}$ prior on the results of Sec. VIC to effectively remove the SHOES likelihood, which itself is effectively a prior on $H_{0}$. The resulting posterior distributions correspond to the fit of the $\Lambda$ CDM or EDE model to Planck 2018 primary CMB, Planck 2018 CMB lensing, BAO data from 6dF, SDSS DR7, and SDSS DR12, Pantheon SNIa, SDSS DR12 RSD, DES-Y1 3x2pt data, and the KiDS and HSC $S_{8}$ priors. Note that the original MCMC sampling for these constraints was performed with the SH0ES likelihood included, which obviates the concerns related to parameter-space volume effects expressed in, e.g., 26]. For a complementary discussion of this issue, see Appendix B of [91].

We find no evidence for the EDE component. The results are shown in Fig. 1 and Fig. 12 for the EDE and standard $\Lambda$ CDM posteriors respectively, and parameter constraints are given in Tables IX and XV. We find an upper bound $f_{\mathrm{EDE}}<0.060$ at $95 \% \mathrm{CL}$; a two-tailed analysis gives $f_{\mathrm{EDE}}=0.023 \pm 0.017$. This constraint is substantially stronger than found with the primary CMB alone (Table II, $f_{\mathrm{EDE}}<0.087$ at $95 \% \mathrm{CL}$ ), and indicates clear tension with the values preferred when including SHOES in the analysis.

We find $H_{0}=68.92_{-0.59}^{+0.57} \mathrm{~km} / \mathrm{s} / \mathrm{Mpc}$, in substantial $(3.3 \sigma)$ tension with SHOES. The EDE value for $H_{0}$ is extremely close to the value found in $\Lambda \mathrm{CDM}$ fit to the same
Constraints from Planck $2018 \mathrm{TT}+\mathrm{TE}+\mathrm{EE}+\mathrm{CMB}$ Lensing, BAO, SNIa, SH0ES, RSD, DES-Y1, KiDS- $S_{8}$, and $\mathrm{HSC}-S_{8}$

\begin{tabular}{|l|c|c|}
\hline \hline Parameter & $\Lambda$ CDM & EDE $(n=3)$ \\
\hline \hline $\boldsymbol{l n}\left(\mathbf{1 0}^{\mathbf{I 0}} \boldsymbol{A}_{\mathbf{s}}\right)$ & $3.046 \pm 0.014$ & $3.053 \pm 0.014$ \\
$\boldsymbol{n}_{\mathbf{s}}$ & $0.9710 \pm 0.0035$ & $0.9814_{-0.0075}^{+0.0075}$ \\
$\mathbf{1 0 0}_{\mathbf{s}}$ & $1.04209 \pm 0.00028$ & $1.04169 \pm 0.00036$ \\
$\boldsymbol{\Omega}_{\mathbf{b}} \boldsymbol{h}^{\mathbf{2}}$ & $0.02260 \pm 0.00013$ & $0.02285 \pm 0.00020$ \\
$\boldsymbol{\Omega}_{\mathbf{c}} \boldsymbol{h}^{\mathbf{2}}$ & $0.11718 \pm 0.00075$ & $0.1230_{-0.0035}^{+0.0034}$ \\
$\boldsymbol{\tau}_{\mathbf{r e i o}}$ & $0.0581 \pm 0.0070$ & $0.0574 \pm 0.0071$ \\
$\log _{\mathbf{1 0}}\left(\boldsymbol{z}_{\boldsymbol{c}}\right)$ & - & $3.73_{-0.19}^{+0.20}$ \\
$\boldsymbol{f}_{\mathbf{E D E}}$ & - & $0.062_{-0.033}^{+0.032}$ \\
$\boldsymbol{\theta}_{\boldsymbol{i}}$ & - & $2.49_{-0.38}^{+0.40}$ \\
\hline$H_{0}[\mathrm{~km} / \mathrm{s} / \mathrm{Mpc}]$ & $68.67 \pm 0.35$ & $70.45_{-1.08}^{+1.05}$ \\
$\Omega_{\mathrm{m}}$ & $0.2978 \pm 0.0044$ & $0.2952^{+0.0046}$ \\
$\sigma_{8}$ & $0.8032 \pm 0.0055$ & $0.8157 \pm 0.0096$ \\
$S_{8}$ & $0.8002 \pm 0.0082$ & $0.8090 \pm 0.0100$ \\
$\log _{10}(\mathrm{f} / \mathrm{eV})$ & - & $26.55_{-0.18}^{+0.13}$ \\
$\log _{10}(\mathrm{~m} / \mathrm{eV})$ & - & $-26.94_{-0.40}^{+0.39}$ \\
\hline
\end{tabular}

TABLE VIII: The mean $\pm 1 \sigma$ constraints on the cosmological parameters in $\Lambda \mathrm{CDM}$ and in the EDE scenario with $n=$ 3, as inferred from the combination of Planck 2018 primary CMB data (TT+TE+EE); Planck 2018 CMB lensing data; BAO data from 6dF, SDSS DR7, and SDSS DR12; Pantheon SNIa data; the latest SH0ES $H_{0}$ constraint; SDSS DR12 RSD data; DES-Y1 3x2pt data; and priors on $S_{8}$ derived from KiDS and HSC data. The KiDS and HSC $S_{8}$ priors serve as approximations to the full likelihood functions for these data sets; we have validated the accuracy of this approximate approach using the DES data, for which we can compare the full likelihood and $S_{8}$-prior approaches, in Fig. 17 . However, we do not provide best-fit parameter values here due to the use of these approximations for the true likelihoods. With the inclusion of the KiDS and HSC $S_{8}$ priors, evidence for the EDE component weakens further beyond that found in Table VI to below $2 \sigma$. This arises from the inability of the EDE model to accommodate the "low" $\sigma_{8}$ and $\Omega_{m}$ values found by the weak lensing experiments. A one-sided upper limit on the EDE fraction yields $f_{\mathrm{EDE}}<0.112$ at $95 \% \mathrm{CL}$.

combination of data sets, $H_{0}=68.33 \pm 0.36 \mathrm{~km} / \mathrm{s} / \mathrm{Mpc}$, consistent with the non-detection of the EDE component in the former.

The posterior distributions for the EDE parameters, Fig. 1, are in broad agreement with those found in the fit to the primary CMB alone. The initial field displacement $\theta_{i}$ is bounded by $\theta_{i}>0.35$ at $95 \% \mathrm{CL}$, comparable to the result from the fit to the primary CMB alone, $\theta_{i}>0.36$. Similarly, we find only a lower bound on $z_{c}$ : $\log _{10}\left(z_{c}\right)>3.28$ at at $95 \% \mathrm{CL}$. The $f_{\text {EDE }}$ posterior is even more weighted towards $f_{\mathrm{EDE}}=0$ than the fit to the CMB alone, as is reflected in the tighter upper bound, $f_{\text {EDE }}<0.060$ at $95 \%$ CL. This upper limit is well below the mean and best-fit values found when including SH0ES and excluding DES, HSC, and KV-450 (see Table IV] or [26]), indicating clear discordance. 


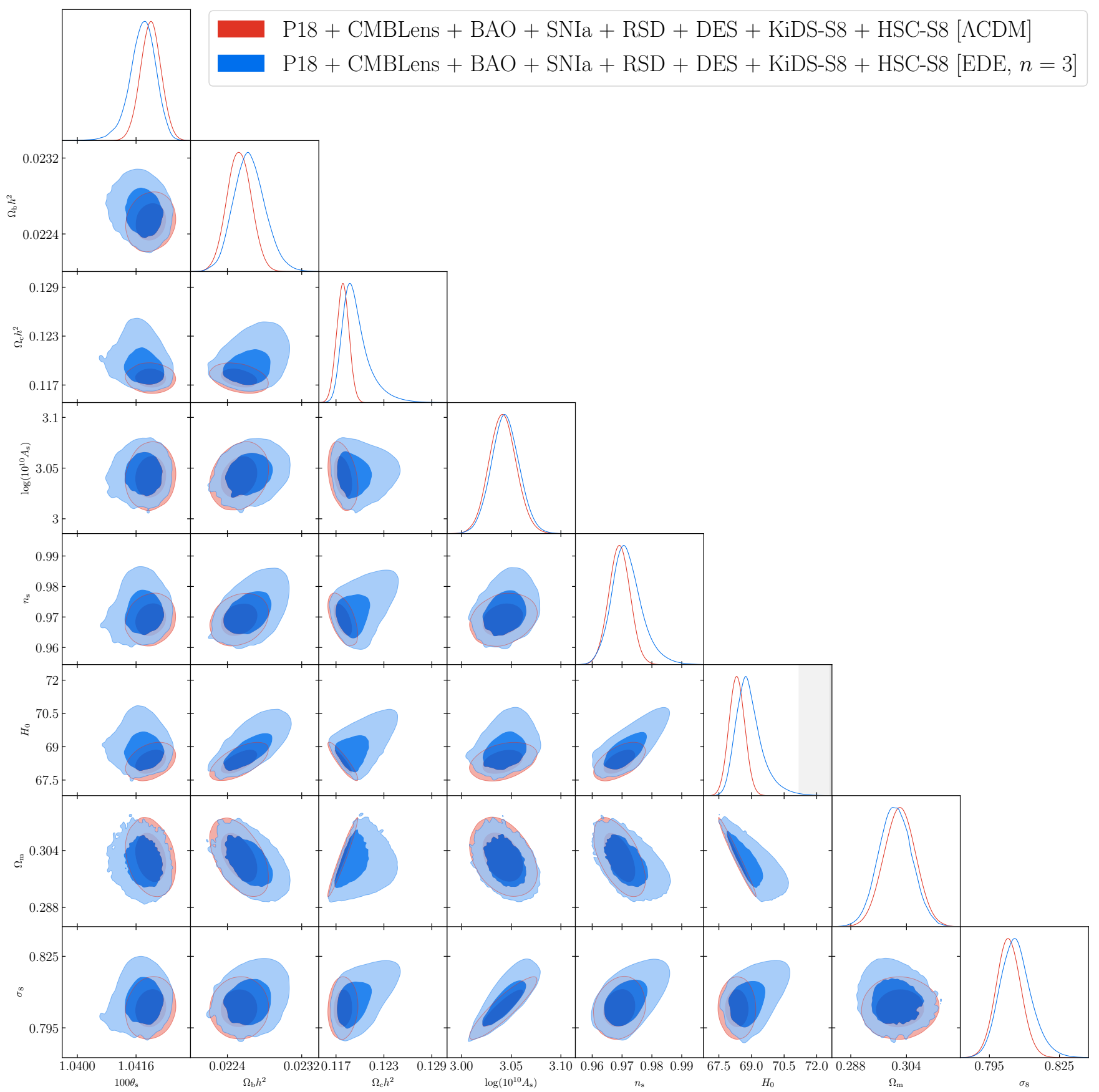

FIG. 12: Cosmological parameter constraints with SH0ES excluded from the combined data set (see Sec. VIE), in $\Lambda$ CDM and EDE. We include Planck 2018 primary CMB data (TT+TE+EE); Planck 2018 CMB lensing data; BAO data from 6dF, SDSS DR7, and SDSS DR12; Pantheon SNIa data; SDSS DR12 RSD data; the DES-Y1 3x2pt data; and KiDS and HSC data approximated by an $S_{8}$ prior. There is little difference in $H_{0}$ between $\Lambda$ CDM and EDE, and the EDE $H_{0}$ posterior has negligible support in the $2 \sigma$ region of the SHOES measurement (indicated by the gray band), indicating that the EDE scenario appears unlikely to resolve the Hubble tension.

\section{PRIOR DEPENDENCE}

All analyses up until this point have followed past work (e.g., 26]) and assumed uniform prior probability distributions for the effective EDE parameters $f_{\mathrm{EDE}}$ and $\log _{10}\left(z_{c}\right)$, as well as for the initial misalignment angle $\theta_{i}$. No consideration has been given to the particle physics parameters, namely the axion mass $m$ and decay constant $f$.

However, implicit assumptions have been made about 
Constraints from Planck $2018 \mathrm{TT}+\mathrm{TE}+\mathrm{EE}+\mathrm{CMB}$ Lensing, $\mathrm{BAO}, \mathrm{SNI}$, RSD, DES-Y1, KiDS- $S_{8}$, and $\mathrm{HSC}-S_{8}$ (No-SH0ES)

\begin{tabular}{|l|c|c|}
\hline \hline Parameter & $\Lambda$ CDM & EDE $(n=3)$ \\
\hline \hline $\boldsymbol{l n}\left(\mathbf{1 0}^{\mathbf{1 0}} \boldsymbol{A}_{\mathbf{s}}\right)$ & $3.041 \pm 0.014$ & $3.044 \pm 0.014$ \\
$\boldsymbol{n}_{\mathbf{s}}$ & $0.9692 \pm 0.0035$ & $0.9718 \pm 0.0049$ \\
$\mathbf{1 0 0}_{\mathbf{s}}$ & $1.04200 \pm 0.00028$ & $1.04177_{-0.00033}^{+0.00035}$ \\
$\boldsymbol{\Omega}_{\mathbf{b}} \boldsymbol{h}^{\mathbf{2}}$ & $0.02253 \pm 0.00013$ & $0.02264 \pm 0.00017$ \\
$\boldsymbol{\Omega}_{\mathbf{c}} \boldsymbol{h}^{\mathbf{2}}$ & $0.11785 \pm 0.00076$ & $0.1196 \pm 0.0016$ \\
$\boldsymbol{\tau}_{\mathbf{r e i o}}$ & $0.0552 \pm 0.0069$ & $0.0558 \pm 0.0069$ \\
$\boldsymbol{l o g}_{\mathbf{1 0}}\left(\boldsymbol{z}_{\boldsymbol{c}}\right)$ & - & $>3.28$ \\
$\boldsymbol{f}_{\mathbf{E D E}}$ & - & $<0.060$ \\
$\boldsymbol{\theta}_{\boldsymbol{i}}$ & - & $>0.35$ \\
\hline$H_{0}[\mathrm{~km} / \mathrm{s} / \mathrm{Mpc}]$ & $68.33 \pm 0.36$ & $68.92_{-0.59}^{+0.57}$ \\
$\Omega_{\mathrm{m}}$ & $0.3021 \pm 0.0045$ & $0.3008 \pm 0.0047$ \\
$\sigma_{8}$ & $0.8032 \pm 0.0053$ & $0.8064 \pm 0.0065$ \\
$S_{8}$ & - & $0.8074 \pm 0.0089$ \\
$\log _{10}(\mathrm{f} / \mathrm{eV})$ & - & $26.52_{-0.36}^{+0.38}$ \\
$\log _{10}(\mathrm{~m} / \mathrm{eV})$ & \multicolumn{2}{|c|}{$-26.67_{-0.69}^{+0.65}$} \\
\hline
\end{tabular}

TABLE IX: The mean $\pm 1 \sigma$ constraints on the cosmological parameters in $\Lambda \mathrm{CDM}$ and in the EDE scenario with $n=3$, as inferred from the combination of Planck 2018 primary CMB data (TT+TE+EE); Planck 2018 CMB lensing data; BAO data from 6dF, SDSS DR7, and SDSS DR12; Pantheon SNIa data; SDSS DR12 RSD data; DES-Y1 3x2pt data; and priors on $S_{8}$ derived from KiDS and HSC data, i.e., all data sets used in TableVIII but with SH0ES excluded. As in that table, we do not provide best-fit parameter values here due to the use of the approximate likelihoods for KiDS and HSC. As in the Planck primary CMB-only analysis (Table II), no evidence for the EDE component is seen here. A two-tailed analysis yields $f_{\mathrm{EDE}}=0.023 \pm 0.017$. The upper limit on $f_{\mathrm{EDE}}$ here is in significant tension with the values preferred when including SHOES in the analysis.

the particle physics parameters: uniform priors on $\left\{f_{\mathrm{EDE}}, \log _{10} z_{c}, \theta_{i}\right\}$, translated into implicit priors on $\left\{f, m, \theta_{i}\right\}$, correspond to strongly non-uniform priors on $f$ and $m$. To illustrate this issue, in Fig. 13 we plot the implicit effective priors imposed on $f$ and $m$ by [26], computed by sampling a uniform probability distribution on $f_{\mathrm{EDE}}, \log _{10}\left(z_{c}\right)$, and $\theta_{i}$, in the range [0.01, 0.25], [3.1, 4.2], and $[0.1,3.0]$, respectively.

One can see in Fig. 13 that the distribution of axion decay constants is highly peaked at $f \sim M_{p l}$, in stark contrast with theory expectations, both from statistical arguments [92, and the Weak Gravity Conjecture 93]; see, e.g., 62. There is a tight correlation between $f$ and $\theta_{i}$, with small $\theta_{i}\left(\theta_{i} \lesssim 1.0\right)$ correlating with superPlanckian decay constants $f>M_{p l}$.

An obvious concern is the dependence of the EDE posterior distributions on the MCMC sampler exploring super-Planckian axion decay constants $\left(f>M_{p l}\right)$, where the theory may no longer be under control. To quantify this effect, we impose an additional prior on the axion decay constant, $f \leq M_{p l}$, on the results of Sec.VIB, i.e., the combined data set that does not include DES, HSC, or KV-450. The posterior distributions are shown in Ap-

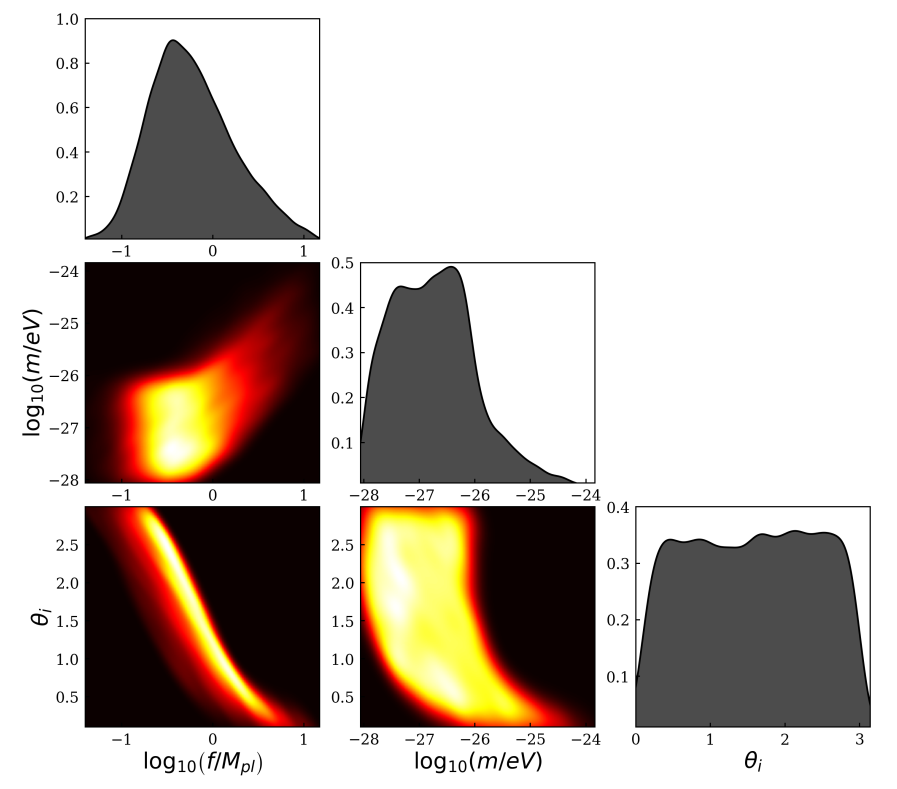

FIG. 13: Effective priors on $f$ and $m$ in EDE with flat priors on $f_{\mathrm{EDE}}, \log _{10}\left(z_{c}\right)$, and $\theta_{i}$. The distribution of $f$ is peaked at $f=0.59 M_{p l}$, where $M_{p l}=2.435 \times 10^{27} \mathrm{eV}$ is the reduced Planck mass.
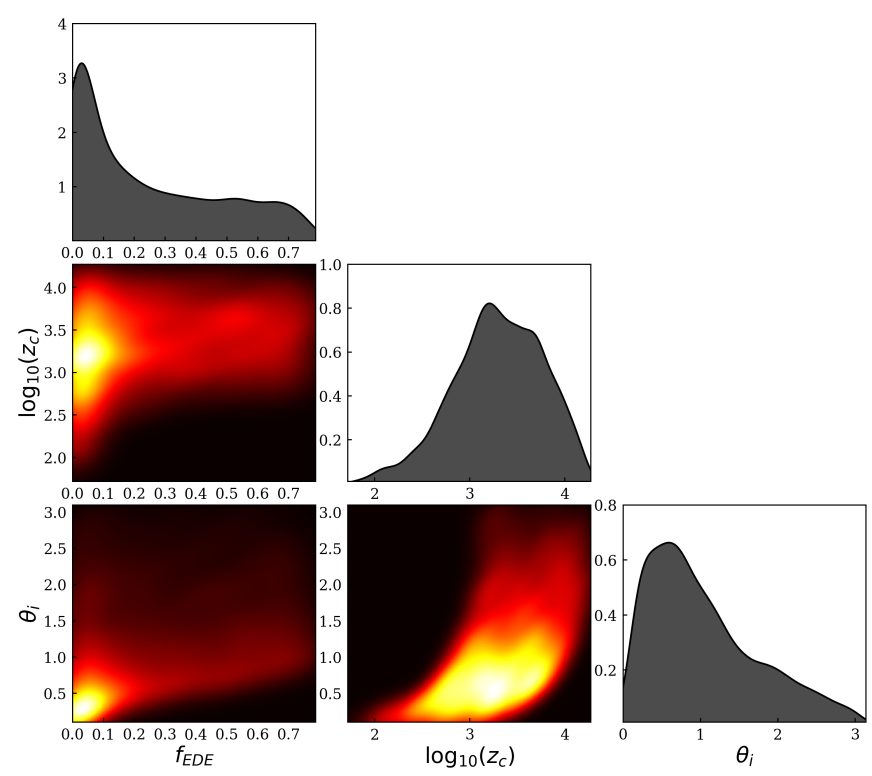

FIG. 14: Effective priors on $f_{\mathrm{EDE}}$ and $\log _{10}\left(z_{c}\right)$ in EDE with flat priors on $f, \log _{10}(m)$, and $\theta_{i}$. The $f_{\mathrm{EDE}}$ distribution is peaked at $f_{\mathrm{EDE}}=0.029$, corresponding to a maximal fraction of $\approx 3 \%$ of the cosmic energy budget in the EDE field. The deviation from a flat distribution of $\theta_{i}$ results from the rejection of samples with $f_{\mathrm{EDE}}>0.8$.

pendix C, Fig. 20. We find the restriction to $f \leq M_{p l}$ has a modest effect, with a small overall impact, including on $f_{\mathrm{EDE}}$.

However, the drastic departure from a flat distribution of decay constants in Fig. 13 raises a more general concern regarding the dependence of the EDE posteriors on 
Constraints from Planck 2018 data only $(\mathrm{TT}+\mathrm{TE}+\mathrm{EE})$ with uniform priors on $f$ and $\log _{10}(m)$

\begin{tabular}{|l|c|}
\hline \hline Parameter & EDE $(n=3)$ \\
\hline \hline $\boldsymbol{l n}\left(\mathbf{1 0}^{\mathbf{1 0}} \boldsymbol{A}_{\mathbf{s}}\right)$ & $3.046(3.042) \pm 0.016$ \\
$\boldsymbol{n}_{\mathbf{s}}$ & $0.9657(0.9626)_{-0.0049}^{+0.0048}$ \\
$\mathbf{1 0 0}_{\mathbf{s}}$ & $1.04177(1.04184) \pm 0.00032$ \\
$\boldsymbol{\Omega}_{\mathbf{b}} \boldsymbol{h}^{\mathbf{2}}$ & $0.02238(0.02222) \pm 0.00017$ \\
$\boldsymbol{\Omega}_{\mathbf{c}} \boldsymbol{h}^{\mathbf{2}}$ & $0.1212(0.1218)_{-0.0019}^{+0.0017}$ \\
$\boldsymbol{\tau}_{\mathbf{r e i o}}$ & $0.0541(0.0532) \pm 0.0075$ \\
$\boldsymbol{f} /\left(\mathbf{1 0}^{\mathbf{2 7}} \mathbf{e V}\right)$ & $2.25(0.80)_{-1.96}^{+2.49}$ \\
$\log _{\mathbf{1 0}}(\boldsymbol{m} / \mathbf{e V})$ & $-26.98(-26.11)_{-0.64}^{+0.66}$ \\
$\boldsymbol{\theta}_{\boldsymbol{i}}$ & $<2.31(0.15)$ \\
\hline$H_{0}[\mathrm{~km} / \mathrm{s} / \mathrm{Mpc}]$ & $67.53(66.63)_{-0.73}^{+0.71}$ \\
$\Omega_{\mathrm{m}}$ & $0.3162(0.3258) \pm 0.0083$ \\
$\sigma_{8}$ & $0.8132(0.8153)_{-0.0089}^{+0.0086}$ \\
$S_{8}$ & $0.8349(0.8497) \pm 0.0166$ \\
$\log _{10}\left(z_{c}\right)$ & $<3.89(3.16)$ \\
$f_{\mathrm{EDE}}$ & $<0.041(0.0003)$ \\
$\log _{10}(f / \mathrm{eV})$ & $27.07(26.92) \pm 0.60$ \\
\hline
\end{tabular}

TABLE X: The mean (best-fit) $\pm 1 \sigma$ constraints on the cosmological parameters in the EDE model with $n=3$, as inferred from Planck 2018 primary CMB data only (TT+TE+EE), with uniform priors placed on the fundamental physics parameter $f$ and $\log _{10}(m)$ rather than the effective EDE parameters $f_{\mathrm{EDE}}$ and $\log _{10}\left(z_{c}\right)$ (as used in all other analyses in this paper, and previously in the literature). Upper limits are quoted at $95 \%$ CL. The upper bound on the EDE component is even tighter than seen in Table II due to the stronger prior weight placed on low values of $f_{\mathrm{EDE}}$ by the physical priors (see Fig. 14).

the choice to impose flat priors on the EDE parameters $f_{\mathrm{EDE}}$ and $\log _{10}\left(z_{c}\right)$ instead of the particle physics parameters $f$ and $m$, the latter arguably being the physically reasonable choice of priors. To further elucidate the prior dependence, we perform an MCMC analysis with uniform priors imposed directly on the particle physics parameters $f$ and $\log _{10}(m)$. We consider $f / \mathrm{eV}=\left[10^{26}, 10^{28}\right]$, $\log _{10}(\mathrm{~m} / \mathrm{eV})=[-26,-28], \theta_{i}=[0.1,3.1]$, and impose an upper bound $f_{\mathrm{EDE}}<0.8$ to ensure a physically reasonable cosmology. We choose to impose a flat prior on $f$ rather than $\log _{10}(f)$ in order to allow a non-negligible probability for large $f_{\mathrm{EDE}}$ values; if we had imposed a flat prior on $\log _{10}(f)$ instead (as one could argue is more physically reasonable), then the results below would even more strongly favor small $f_{\mathrm{EDE}}$ values. To illustrate the difference in priors, in Fig. 14 we plot the implied prior probability distributions for $f_{\mathrm{EDE}}$ and $z_{c}$. From this one can appreciate that uniform priors on $f$ and $\log _{10}(m)$ impose a strong prior preference for small $f_{\mathrm{EDE}}$ values, $f_{\mathrm{EDE}}<0.1$, and the distribution is peaked at $f_{\mathrm{EDE}}=0.029$. The $\theta_{i}$ distribution deviates significantly from a flat distribution, due solely to the restriction to samples with $f_{\mathrm{EDE}}<0.8$.

As a first analysis of the effect of these physical priors, we consider the fit to primary CMB data alone. We recompute the EDE parameter constraints of Sec. VIA with the above uniform priors imposed on $f$ and $\log _{10}(m)$. The posterior distributions are shown in Fig. 15 for the EDE parameters and Fig. 9 for the standard $\Lambda$ CDM parameters. The parameter constraints are tabulated in Table $\mathrm{X}$.

The upper bound on the EDE fraction is even stronger than found in Sec. VIA, which further exacerbates the discrepancy of EDE fit to the primary $\mathrm{CMB}$ with the fit of EDE to primary CMB, CMB lensing, BAO, RSD, SNIa, and SHOES (Sec. VIB). We find $f_{\mathrm{EDE}}<0.041$ at $95 \% \mathrm{CL}$; the best-fit model lies nearly exactly at $f_{\mathrm{EDE}}=0$. The posterior distribution for $\theta_{i}$ in Fig. 15 is heavily skewed towards $\theta_{i}=0$, which is strongly correlated with small $f_{\mathrm{EDE}}$ (see Fig. 14). At small values of $f_{\mathrm{EDE}}$ the model is poorly constrained, and we observe this in the posteriors of $f$ and $m$, which are visibly prior-dominated. This is despite the fact that the priors imposed on $\log _{10}(m)$ and $f$ encompass the peak values found with uniform priors on $f_{\mathrm{EDE}}$ and $\log _{10}\left(z_{c}\right)$ in Sec. VIA. In turn, the best-fit parameter values given in Table $\mathrm{X}$ reflect the posterior preference for small $f_{\mathrm{EDE}}$ : while the true maximum of the likelihood should be near the best-fit parameter values quoted in Table II] any reasonable numerical search for a maximum will converge on $f_{\mathrm{EDE}} \simeq 0$ here, and not on $f_{\mathrm{EDE}}=0.068$ as quoted in Table II, due to the posteriors' strong weight toward small $f_{\mathrm{EDE}}$ values in this analysis.

The posterior distributions for the standard $\Lambda$ CDM parameters in the EDE scenario (green contours in Fig. 9), are nearly identical to the $\Lambda$ CDM contours (red). The only hint of EDE is in a slight broadening of the $\Lambda \mathrm{CDM}$ parameter posteriors. The slight shift in $H_{0}$ observed in Sec. VIA is absent, and we find $H_{0}$ is nearly identical to that in $\Lambda \mathrm{CDM}$.

\section{DISCUSSION AND CONCLUSIONS}

The Hubble tension (e.g., [1] poses a challenge for precision cosmology. While systematic errors in the calibration of the cosmic distance ladder and/or other data sets may be the ultimate explanation, the growing severity of the tension (modulo the recent TRGB result in [16]) provides impetus to examine theoretical explanations and explore alternative cosmologies that may restore cosmological concordance.

A logical possibility is the presence of new physics in the early universe. One well-motivated scenario is the introduction of a new source of energy density that increases $H(z)$ just prior to recombination, decreasing the sound horizon, and thereby raising the value of $H_{0}$ inferred from early-universe probes. A popular proposal of this type is the EDE scenario [25], and its variations 26 33.

In this work we reanalyze the EDE scenario accounting for Planck 2018 primary CMB data (TT+TE+EE) and LSS data from Planck 2018 CMB lensing, BAO (6dF, SDSS DR7, and SDSS DR12), SDSS DR12 RSD, DES-Y1 $3 \mathrm{x} 2 \mathrm{pt}$, HSC, and KV-450, as well as supernova distance 

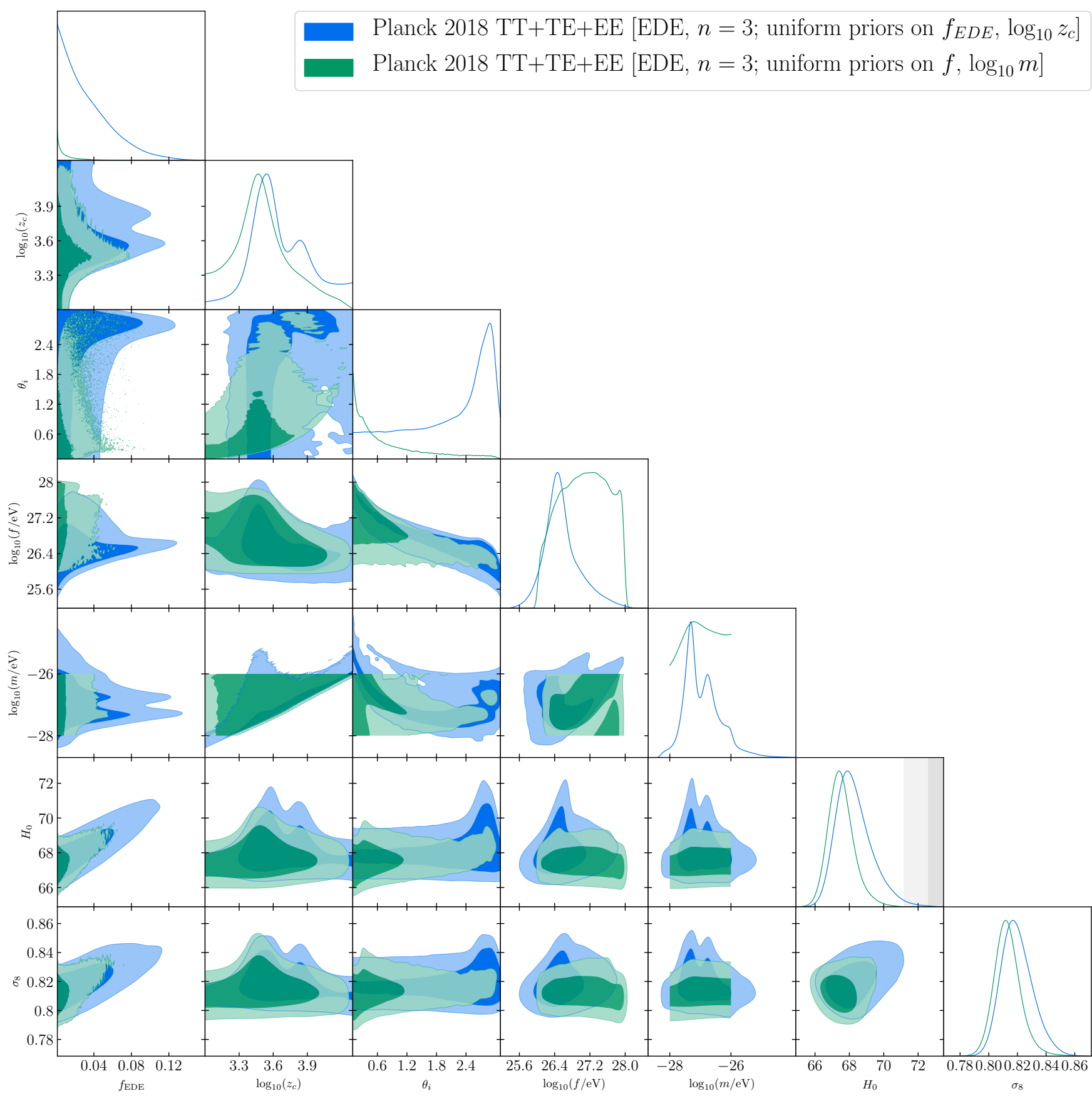

FIG. 15: Constraints on the EDE scenario from Planck 2018 primary CMB data alone, with varying choices of priors on the EDE model parameters. The blue contours show results with uniform priors placed on the effective EDE parameters $f_{\mathrm{EDE}}$ and $\log _{10}\left(z_{c}\right)$, while the green contours show results with uniform priors placed on the fundamental physics EDE parameters $f$ and $\log _{10}(m)$. The physical priors strongly downweight large $f_{\text {EDE }}$ values, and thus significantly disfavor large $H_{0}$ values. The possibility of resolving the tension with SH0ES (gray bands) is thus significantly weakened when adopting these physical priors, rather than uniform priors on the effective EDE parameters.

data from the Pantheon compilation and the SH0ES distance-ladder $H_{0}$ measurement. We obtain strong constraints on the existence of an EDE component in the early universe, as seen in Fig. 1 and Table [.

In the region of parameter space capable of address- ing the Hubble tension, the impact of EDE on LSS observables is substantial. To quantify and contextualize this, in Fig. 8 we consider the imprint of EDE models on the matter power spectrum in the range of wavenumbers probed by DES, in comparison to the matter power spec- 
trum inferred from DES-Y1 measurements 38. In Fig. 6 and Fig. 7 we show the fractional change in the matter power spectrum as a function of the effective EDE parameters $f_{\mathrm{EDE}}$ and $z_{c}$, corresponding to the amount and timing of EDE. For $f_{\mathrm{EDE}} \approx 12 \%$, as proposed in past works, the increase in $P(k)$ is $\mathcal{O}(10 \%)$ on small scales, in particular those that are probed by the DES, HSC, and KV-450 data sets. This change is primarily driven by the significant shifts in the standard $\Lambda$ CDM parameters (especially $\omega_{\text {cdm }}$ and $n_{s}$ ) that are seen when fitting the CMB and SHOES data to the EDE model, although the scale-dependent suppression of growth induced by the EDE itself also affects the shape of $P(k)$.

Our main results are given in Fig. 1 and Table I. We find no evidence for EDE in the primary CMB anisotropies alone: the fit to Planck 2018 TT+TE+EE gives an upper bound $f_{\mathrm{EDE}}<0.087$ at $95 \% \mathrm{CL}$, with $H_{0}=68.29_{-1.00}^{+1.02} \mathrm{~km} / \mathrm{s} / \mathrm{Mpc}$, shifted only slightly upwards relative to the $\Lambda \mathrm{CDM}$ value $H_{0}=67.29 \pm$ $0.59 \mathrm{~km} / \mathrm{s} / \mathrm{Mpc}$, and with a considerably larger error bar. Both $H_{0}$ values are in significant tension with the SH0ES measurement $H_{0}=74.03 \pm 1.42 \mathrm{~km} / \mathrm{s} / \mathrm{Mpc}$ [15]. When the primary $\mathrm{CMB}$ data set is supplemented with data from Planck 2018 CMB lensing, BAO distance measurements, RSD data, the Pantheon SNIa distance measurements, and the SHOES $H_{0}$ measurement, we instead find $3.1 \sigma$ evidence for EDE, $f_{\mathrm{EDE}}=0.098 \pm 0.032$. We find $H_{0}=70.98 \pm 1.05 \mathrm{~km} / \mathrm{s} / \mathrm{Mpc}$, reducing the tension with SH0ES to $\approx 1.7 \sigma$. This is consistent with past results in the literature for this combination of data sets [25, 26].

However, this is not the end of the story: these shifts in $f_{\mathrm{EDE}}$ and $H_{0}$ are reversed upon the inclusion of additional LSS data, in particular measurements of weak gravitational lensing and galaxy clustering. These data strongly constrain $S_{8}$, which in the EDE scenario is highly correlated with $f_{\mathrm{EDE}}$ and $H_{0}$. The tight correlation between $\sigma_{8}, f_{\mathrm{EDE}}$, and $H_{0}$ is clearly visible in the posterior distributions in Fig. 1, and is manifested in the matter power spectrum. We find that additional LSS data substantially weaken the evidence for EDE, as a result of the tension between the larger values of $S_{8}$ needed to fit the CMB and SH0ES in these models and the lower values of this parameter measured in LSS surveys. Including the full DES-Y1 3x2pt likelihoods, we find the evidence for EDE is reduced to $2.3 \sigma$, with $f_{\mathrm{EDE}}=0.077_{-0.034}^{+0.032}$. The inclusion of HSC and $\mathrm{KV}$ 450 data, approximated as priors on $S_{8}$, shrinks this further to $f_{\mathrm{EDE}}=0.062_{-0.033}^{+0.032}$, corresponding to a onesided upper bound $f_{\mathrm{EDE}}<0.112$ at $95 \% \mathrm{CL}$. We find $H_{0}=70.45_{-1.08}^{+1.05}$ in the fit with DES, HSC, and KV-450 included, in moderate $2 \sigma$ tension with SH0ES alone (note that SH0ES is included in all of the aforementioned fits). There is no sign of concordance amongst these data sets: the LSS data pull the parameters in the opposite direction to that required to simultaneously fit the CMB and SH0ES data.

To understand the apparent conflict between LSS data and SHOES-tension-resolving EDE cosmologies, we fit the EDE model to the combined data set with SH0ES excluded in Sec. VIE (note that Pantheon SNIa relative distances are still included). We find a constraint on EDE even tighter than found with the primary CMB alone, $f_{\mathrm{EDE}}<0.060$ at $95 \% \mathrm{CL}$, with no hint of a preference for EDE. The corresponding Hubble constraint is $H_{0}=68.92_{-0.59}^{+0.57} \mathrm{~km} / \mathrm{s} / \mathrm{Mpc}$, in significant tension with SH0ES. The tight constraint found here indicates that CMB and LSS data do not show any hint of moving toward a cosmology that can accommodate the SHOES $H_{0}$ value, even in the broadened EDE parameter space. Physically, this arises from the fact that a higher $H_{0}$ value in the CMB fit requires a higher $f_{\mathrm{EDE}}$ value, which in turn requires higher $\omega_{\mathrm{cdm}}$ and $n_{s}$ values, thereby increasing $\sigma_{8}$ and conflicting with LSS constraints. There does not appear to be a viable swath of parameter space to satisfy all existing constraints.

Finally, we examine the choice of priors and the role of the axion decay constant $f$. Uniform priors imposed on $f_{\mathrm{EDE}}, \log _{10} z_{c}$, and $\theta_{i}$ effectively impose a non-uniform prior on $f$ and $\log _{10}(m)$, as seen in Fig. 13 . Notably, the effective prior for the axion decay constant $f$ is peaked near the Planck scale, $f \approx M_{p l}$, in conflict with theoretical expectations from particle physics and quantum gravity. When the fit to primary $\mathrm{CMB}$ data is repeated with uniform priors imposed directly on $f$ and $\log _{10}(m)$, the upper bound on $f_{\mathrm{EDE}}$ becomes $f_{\mathrm{EDE}}<0.041$ at $95 \%$ CL. This is significantly lower than the corresponding result for uniform priors on the effective parameters $f_{\mathrm{EDE}}$ and $z_{c}$, suggestive of a prior dependence for EDE results more generally. The use of such physical priors in the other analyses presented in this paper would only further tighten the upper limits on EDE.

Taken in conjunction, these results paint a bleak picture for the viability of the EDE scenario as a candidate to restore cosmological concordance. More generally, it is likely that any model that attempts to decrease the sound horizon by increasing $H(z)$ through a new darkenergy-like component that is active at early times will encounter the problems identified here. All such models, insofar as they can accommodate a close fit to both the CMB and SHOES measurement, will do so at the cost of a shift in $\Lambda$ CDM parameters that is not compatible with LSS data. Furthermore, we have not utilized all possible data sets that constrain LSS in this paper; in particular, galaxy cluster number counts (e.g., 94 97]) and thermal Sunyaev-Zel'dovich power spectrum measurements (e.g., 98, 99]), amongst other probes, tightly constrain $\sigma_{8}$ and $\Omega_{m}$. Cluster number counts tend to favor "low" values of $\sigma_{8}$, consistent with weak lensing and other LSS probes. Thus, their inclusion would likely strengthen the conclusions drawn here. However, it may be important to explicitly verify the accuracy of current fitting functions (e.g., 100) or emulators that are used for the halo mass function in the context of EDE cosmologies, prior to applying this methodology to constrain the EDE scenario.

Broadening the model space, one possible solution to 
the tensions identified in this work may be to introduce a larger neutrino mass, which would suppress small-scale power in $P(k)$ and thereby allow larger $f_{\mathrm{EDE}}$ (and hence $H_{0}$ ) than found here. However, whether a large enough neutrino mass is consistent with the $\mathrm{CMB}$ and distance probes is unclear. The coming decade will see the launch of several powerful LSS experiments (e.g., WFIRST [52], DESI [49, VRO [50] (formerly LSST), and Euclid [51]), and with these, an abundance of data from a range of redshifts. In the absence of significant shifts with respect to current LSS data, it seems unlikely that these nextgeneration data sets will reverse the negative trajectory we have seen here in the evidence for EDE as LSS data are included in the analysis. However, the additional statistical power will allow for tight constraints on EDE, even when additional degrees of freedom are allowed to vary (e.g., neutrino masses, $N_{\text {eff }}$, the primordial power spectrum, etc.).

Regardless of implications for the Hubble tension, ultralight axions are of cosmological interest in their own right (see, e.g., 101-103), and the EDE variant of this idea may leave interesting cosmological signatures even in the region of parameter space where the impact on the inferred $H_{0}$ value is minimal. For example, interesting effects arise in birefringence of $\mathrm{CMB}$ polarization
104, in principle yielding a signal in CMB circular polarization [105, 106]. Other interesting signals may arise due to the parametric resonance effects described in [26]. Orthogonal to these considerations, it may be interesting to perform an appraisal of the discordance of the EDE model along the lines proposed in, e.g., 84 86. Looking towards alternative approaches to the $H_{0}$ tension, the results presented here potentially motivate further study of new physics in the cosmic distance ladder itself [107 109]. We leave these interesting directions to future work.

\section{Acknowledgments}

The authors thank Eric Baxter, Jo Dunkley, Daniel Foreman-Mackey, Gil Holder, Mikhail Ivanov, Antony Lewis, Julien Lesgourgues, Blake Sherwin, Marko Simonovic, David Spergel, and Matias Zaldarriaga for helpful discussions, as well as the Scientific Computing Core staff at the Flatiron Institute for computational support. EM and MT thank the Simons Foundation and the Flatiron Institute Center for Computational Astrophysics for hospitality while a portion of this work was completed. JCH thanks the Simons Foundation for support.
[1] L. Verde, T. Treu and A. G. Riess, Tensions between the Early and the Late Universe, in Nature Astronomy 2019, 2019, 1907.10625, DOI

[2] G. Hinshaw, D. Larson, E. Komatsu, D. N. Spergel, C. L. Bennett, J. Dunkley et al., Nine-year Wilkinson Microwave Anisotropy Probe (WMAP) Observations: Cosmological Parameter Results, ApJS 208 (2013) 19 1212.5226 .

[3] Planck collaboration, P. A. R. Ade et al., Planck 2013 results. XVI. Cosmological parameters, Astron. Astrophys. 571 (2014) A16 1303.5076.

[4] Planck collaboration, P. A. R. Ade et al., Planck 2015 results. XIII. Cosmological parameters, Astron. Astrophys. 594 (2016) A13 1502.01589.

[5] Planck collaboration, N. Aghanim et al., Planck 2018 results. VI. Cosmological parameters, 1807.06209

[6] ACTPol collaboration, T. Louis et al., The Atacama Cosmology Telescope: Two-Season ACTPol Spectra and Parameters, JCAP 1706 (2017) 031 1610.02360.

[7] SPT collaboration, F. Bianchini et al., Constraints on Cosmological Parameters from the $500 \mathrm{deg}^{2}$ SPTpol Lensing Power Spectrum, 1910.07157.

[8] R. J. Cooke, M. Pettini, K. M. Nollett and R. Jorgenson, The Primordial Deuterium Abundance of the Most Metal-poor Damped Lyman- $\alpha$ System, ApJ 830 (2016) 148 1607.03900.

[9] D. J. Fixsen, E. S. Cheng, J. M. Gales, J. C. Mather, R. A. Shafer and E. L. Wright, The Cosmic Microwave Background Spectrum from the Full COBE FIRAS Data Set, ApJ 473 (1996) 576 astro-ph/9605054.

[10] DES collaboration, T. M. C. Abbott et al., Dark Energy Survey Year 1 Results: A Precise HO Estimate from DES Y1, BAO, and D/H Data, Mon. Not. Roy. Astron. Soc. 480 (2018) 38791711.00403.

[11] M. M. Ivanov, M. Simonović and M. Zaldarriaga, Cosmological Parameters and Neutrino Masses from the Final Planck and Full-Shape BOSS Data, 1912.08208 .

[12] G. D'Amico, J. Gleyzes, N. Kokron, D. Markovic, L. Senatore, P. Zhang et al., The Cosmological Analysis of the SDSS/BOSS data from the Effective Field Theory of Large-Scale Structure, 1909.05271

[13] T. Tröster, A. G. Sánchez, M. Asgari, C. Blake, M. Crocce, C. Heymans et al., Cosmology from large-scale structure. Constraining $\Lambda$ CDM with BOSS, A\&A 633 (2020) L10 1909.11006.

[14] A. Sandage, G. A. Tammann, A. Saha, B. Reindl, F. D. Macchetto and N. Panagia, The Hubble Constant: A Summary of the Hubble Space Telescope Program for the Luminosity Calibration of Type Ia Supernovae by Means of Cepheids, ApJ 653 (2006) 843 astro-ph/0603647.

[15] A. G. Riess, S. Casertano, W. Yuan, L. M. Macri and D. Scolnic, Large Magellanic Cloud Cepheid Standards Provide a $1 \%$ Foundation for the Determination of the Hubble Constant and Stronger Evidence for Physics beyond $\Lambda C D M$, Astrophys. J. 876 (2019) 85 1903.07603.

[16] W. L. Freedman, B. F. Madore, T. Hoyt, I. S. Jang, R. Beaton, M. G. Lee et al., Calibration of the Tip of the Red Giant Branch (TRGB), 2002.01550.

[17] C. D. Huang, A. G. Riess, W. Yuan, L. M. Macri, N. L. Zakamska, S. Casertano et al., Hubble Space Telescope Observations of Mira Variables in the Type 
Ia Supernova Host NGC 1559: An Alternative Candle to Measure the Hubble Constant, 1908.10883.

[18] K. C. Wong et al., HOLiCOW XIII. A 2.4\% measurement of $H_{0}$ from lensed quasars: $5.3 \sigma$ tension between early and late-Universe probes, 1907.04869.

[19] C. S. Kochanek, Over-constrained Gravitational Lens Models and the Hubble Constant, Mon. Not. Roy. Astron. Soc. 493 (2020) 1725 1911.05083.

[20] K. Blum, E. Castorina and M. Simonović, Could quasar lensing time delays hint to cored dark matter halos, instead of $H_{0}$ tension?, 2001.07182

[21] D. W. Pesce et al., The Megamaser Cosmology Project. XIII. Combined Hubble constant constraints, Astrophys. J. 891 (2020) L1 2001.09213.

[22] LigO Scientific, Virgo, 1M2H, DARK Energy Camera GW-E, DES, DLT40, Las Cumbres OBSERVATORY, VINROUGE, MASTER collaboration, B. P. Abbott et al., A gravitational-wave standard siren measurement of the Hubble constant, Nature 551 (2017) 851710.05835.

[23] DES, LIGO ScIENTIFIC, VIRGO collaboration, M. Soares-Santos et al., First Measurement of the Hubble Constant from a Dark Standard Siren using the Dark Energy Survey Galaxies and the LIGO/Virgo Binary-Black-hole Merger GW170814, Astrophys. J. 876 (2019) L7 1901.01540.

[24] L. Knox and M. Millea, Hubble constant hunter's guide, Phys. Rev. D101 (2020) 043533 1908.03663.

[25] V. Poulin, T. L. Smith, T. Karwal and M. Kamionkowski, Early Dark Energy Can Resolve The Hubble Tension, Phys. Rev. Lett. 122 (2019) 221301 1811.04083.

[26] T. L. Smith, V. Poulin and M. A. Amin, Oscillating scalar fields and the Hubble tension: a resolution with novel signatures, Phys. Rev. D101 (2020) 063523 1908.06995.

[27] P. Agrawal, F.-Y. Cyr-Racine, D. Pinner and L. Randall, Rock 'n' Roll Solutions to the Hubble Tension, 1904.01016

[28] S. Alexander and E. McDonough, Axion-Dilaton Destabilization and the Hubble Tension, Phys. Lett. B797 (2019) 1348301904.08912.

[29] M.-X. Lin, G. Benevento, W. Hu and M. Raveri, Acoustic Dark Energy: Potential Conversion of the Hubble Tension, Phys. Rev. D100 (2019) 063542 1905.12618 .

[30] J. Sakstein and M. Trodden, Early dark energy from massive neutrinos - a natural resolution of the Hubble tension, 1911.11760

[31] F. Niedermann and M. S. Sloth, New Early Dark Energy, 1910.10739.

[32] N. Kaloper, Dark Energy, $H_{0}$ and Weak Gravity Conjecture, Int. J. Mod. Phys. D28 (2019) 1944017 1903.11676.

[33] K. V. Berghaus and T. Karwal, Thermal Friction as a Solution to the Hubble Tension, 1911.06281.

[34] M. Kamionkowski, J. Pradler and D. G. E. Walker, Dark energy from the string axiverse, Phys. Rev. Lett. 113 (2014) 2513021409.0549.

[35] V. Poulin, T. L. Smith, D. Grin, T. Karwal and M. Kamionkowski, Cosmological implications of ultralight axionlike fields, PhRvD 98 (2018) 083525 1806.10608.

[36] J. C. Hill and E. J. Baxter, Can early dark energy explain EDGES?, JCAP 2018 (2018) 037 1803.07555.

[37] A. Lewis, GetDist: a Python package for analysing Monte Carlo samples, arXiv e-prints (2019) arXiv:1910.13970 1910.13970.

[38] DES collaboration, T. M. C. Abbott et al., Dark Energy Survey year 1 results: Cosmological constraints from galaxy clustering and weak lensing, Phys. Rev. D98 (2018) 043526 1708.01530.

[39] K. Dutta, A. Roy, Ruchika, A. A. Sen and M. M. Sheikh-Jabbari, Cosmology with low-redshift observations: No signal for new physics, Phys. Rev. D100 (2019) 103501 1908.07267.

[40] C. Krishnan, E. O. Colgain, Ruchika, A. A. Sen, M. Sheikh-Jabbari and T. Yang, Is there an early Universe solution to Hubble tension?, 2002.06044

[41] BOSS collaboration, S. Alam et al., The clustering of galaxies in the completed SDSS-III Baryon Oscillation Spectroscopic Survey: cosmological analysis of the DR12 galaxy sample, Mon. Not. Roy. Astron. Soc. 470 (2017) 26171607.03155 .

[42] H. Hildebrandt et al., KiDS-450: Cosmological parameter constraints from tomographic weak gravitational lensing, Mon. Not. Roy. Astron. Soc. 465 (2017) 1454 1606.05338.

[43] H. Hildebrandt, F. Köhlinger, J. L. van den Busch, B. Joachimi, C. Heymans, A. Kannawadi et al., KiDS+VIKING-450: Cosmic shear tomography with optical and infrared data, A\&A 633 (2020) A69 1812.06076 .

[44] HSC collaboration, C. Hikage et al., Cosmology from cosmic shear power spectra with Subaru Hyper Suprime-Cam first-year data, Publ. Astron. Soc. Jap. 71 (2019) Publications of the Astronomical Society of Japan, Volume 71, Issue 2, April 2019, 43, https://doi.org/10.1093/pasj/psz010 [1809.09148].

[45] J. J. M. Carrasco, M. P. Hertzberg and L. Senatore, The Effective Field Theory of Cosmological Large Scale Structures, JHEP 09 (2012) 082 1206.2926.

[46] D. Baumann, A. Nicolis, L. Senatore and M. Zaldarriaga, Cosmological Non-Linearities as an Effective Fluid, JCAP 1207 (2012) 051 1004.2488.

[47] T. Colas, G. D'amico, L. Senatore, P. Zhang and F. Beutler, Efficient Cosmological Analysis of the SDSS/BOSS data from the Effective Field Theory of Large-Scale Structure, 1909.07951.

[48] O. H. E. Philcox, M. M. Ivanov, M. Simonović and M. Zaldarriaga, Combining Full-Shape and BAO Analyses of Galaxy Power Spectra: A 1.6\% $C M B$-independent constraint on HO, 2002.04035

[49] DESI collaboration, M. E. Levi et al., The Dark Energy Spectroscopic Instrument (DESI), 1907.10688

[50] LSST collaboration, Z. Ivezic et al., LSST: from Science Drivers to Reference Design and Anticipated Data Products, Astrophys. J. 873 (2019) 111 0805.2366.

[51] L. Amendola et al., Cosmology and fundamental physics with the Euclid satellite, Living Rev. Rel. 21 (2018) 2 1606.00180.

[52] R. Akeson, L. Armus, E. Bachelet, V. Bailey, L. Bartusek, A. Bellini et al., The Wide Field Infrared Survey Telescope: 100 Hubbles for the 2020s, arXiv e-prints (2019) arXiv:1902.05569 1902.05569.

[53] J. C. Hill, E. McDonough and M. W. Toomey, 
Class-ede, Mar, 2020.

[54] P. Ade, J. Aguirre, Z. Ahmed, S. Aiola, A. Ali, D. Alonso et al., The Simons Observatory: science goals and forecasts, JCAP 2019 (2019) 056 1808.07445 .

[55] K. Abazajian, G. Addison, P. Adshead, Z. Ahmed, S. W. Allen, D. Alonso et al., CMB-S4 Science Case, Reference Design, and Project Plan, arXiv e-prints (2019) arXiv:1907.04473 1907.04473.

[56] R. D. Peccei and H. R. Quinn, CP Conservation in the Presence of Instantons, Phys. Rev. Lett. 38 (1977) 1440.

[57] F. Wilczek, Problem of Strong $P$ and T Invariance in the Presence of Instantons, Phys. Rev. Lett. 40 (1978) 279 .

[58] S. Weinberg, A New Light Boson?, Phys. Rev. Lett. 40 (1978) 223

[59] M. Montero, A. M. Uranga and I. Valenzuela, Transplanckian axions!?, JHEP 08 (2015) 032 1503.03886 .

[60] T. Rudelius, Constraints on Axion Inflation from the Weak Gravity Conjecture, JCAP 1509 (2015) 020 1503.00795.

[61] T. Banks, M. Dine, P. J. Fox and E. Gorbatov, On the possibility of large axion decay constants, JCAP 0306 (2003) 001 hep-th/0303252.

[62] T. Rudelius, On the Possibility of Large Axion Moduli Spaces, JCAP 1504 (2015) 049 1409.5793.

[63] M. S. Turner, Coherent scalar-field oscillations in an expanding universe, Phys. Rev. D 28 (1983) 1243.

[64] J. Lesgourgues, The Cosmic Linear Anisotropy Solving System (CLASS) I: Overview, arXiv e-prints (2011) arXiv:1104.2932 1104.2932.

[65] D. Blas, J. Lesgourgues and T. Tram, The Cosmic Linear Anisotropy Solving System (CLASS). Part II: Approximation schemes, JCAP 2011 (2011) 034 1104.2933.

[66] VIRGO Consortium collaboration, R. E. Smith, J. A. Peacock, A. Jenkins, S. D. M. White, C. S. Frenk, F. R. Pearce et al., Stable clustering, the halo model and nonlinear cosmological power spectra, Mon. Not. Roy. Astron. Soc. 341 (2003) 1311 astro-ph/0207664.

[67] R. Takahashi, M. Sato, T. Nishimichi, A. Taruya and M. Oguri, Revising the Halofit Model for the Nonlinear Matter Power Spectrum, ApJ 761 (2012) 152 1208.2701.

[68] J. Torrado and A. Lewis, Cobaya, Oct, 2019.

[69] A. Lewis and S. Bridle, Cosmological parameters from $C M B$ and other data: A Monte Carlo approach, PhRvD 66 (2002) 103511 astro-ph/0205436.

[70] A. Lewis, Efficient sampling of fast and slow cosmological parameters, PhRvD 87 (2013) 103529 1304.4473 .

[71] R. M. Neal, Taking Bigger Metropolis Steps by Dragging Fast Variables, arXiv Mathematics e-prints (2005) math/0502099 math/0502099.

[72] A. Gelman and D. B. Rubin, Inference from Iterative Simulation Using Multiple Sequences, Statist. Sci. 7 (1992) 457.

[73] M. Powell, The bobyqa algorithm for bound constrained optimization without derivatives, Technical Report, Department of Applied Mathematics and Theoretical Physics (2009).
[74] C. Cartis, J. Fiala, B. Marteau and L. Roberts, Improving the Flexibility and Robustness of Model-Based Derivative-Free Optimization Solvers, arXiv e-prints (2018) arXiv:1804.00154 1804.00154.

[75] C. Cartis, L. Roberts and O. Sheridan-Methven, Escaping local minima with derivative-free methods: a numerical investigation, arXiv e-prints (2018) arXiv:1812.11343 1812.11343.

[76] Planck Collaboration, N. Aghanim, Y. Akrami, M. Ashdown, J. Aumont, C. Baccigalupi et al., Planck 2018 results. V. CMB power spectra and likelihoods, arXiv e-prints (2019) arXiv:1907.12875 1907.12875.

[77] Planck Collaboration, N. Aghanim, Y. Akrami, M. Ashdown, J. Aumont, C. Baccigalupi et al., Planck 2018 results. VIII. Gravitational lensing, arXiv e-prints (2018) arXiv:1807.06210 1807.06210.

[78] Planck Collaboration, N. Aghanim, M. Arnaud, M. Ashdown, J. Aumont, C. Baccigalupi et al., Planck 2015 results. XI. CMB power spectra, likelihoods, and robustness of parameters, A\&A 594 (2016) A11 1507.02704 .

[79] A. J. Ross, L. Samushia, C. Howlett, W. J. Percival, A. Burden and M. Manera, The clustering of the SDSS DR7 main Galaxy sample - I. A 4 per cent distance measure at $z=0.15$, Mon. Not. Roy. Astron. Soc. 449 (2015) 835 1409.3242.

[80] F. Beutler, C. Blake, M. Colless, D. H. Jones, L. Staveley-Smith, L. Campbell et al., The 6dF Galaxy Survey: baryon acoustic oscillations and the local Hubble constant, MNRAS 416 (2011) 3017 1106.3366.

[81] BOSS collaboration, S. Satpathy et al., The clustering of galaxies in the completed SDSS-III Baryon Oscillation Spectroscopic Survey: On the measurement of growth rate using galaxy correlation functions, Mon. Not. Roy. Astron. Soc. 469 (2017) 1369 [1607.03148.

[82] N. Kaiser, Clustering in real space and in redshift space, MNRAS 227 (1987) 1

[83] D. M. Scolnic et al., The Complete Light-curve Sample of Spectroscopically Confirmed SNe Ia from Pan-STARRS1 and Cosmological Constraints from the Combined Pantheon Sample, Astrophys. J. 859 (2018) 1011710.00845.

[84] M. Raveri and W. Hu, Concordance and Discordance in Cosmology, Phys. Rev. D99 (2019) 043506 1806.04649 .

[85] M. Raveri, G. Zacharegkas and W. Hu, Quantifying concordance of correlated cosmological data sets, 1912.04880

[86] P. Lemos, F. Köhlinger, W. Handley, B. Joachimi, L. Whiteway and O. Lahav, Quantifying Suspiciousness Within Correlated Data Sets, 1910.07820

[87] A. Drlica-Wagner, I. Sevilla-Noarbe, E. S. Rykoff, R. A. Gruendl, B. Yanny, D. L. Tucker et al., Dark Energy Survey Year 1 Results: The Photometric Data Set for Cosmology, ApJS 235 (2018) 33 1708.01531.

[88] R. Mandelbaum, H. Miyatake, T. Hamana, M. Oguri, M. Simet, R. Armstrong et al., The first-year shear catalog of the Subaru Hyper Suprime-Cam Subaru Strategic Program Survey, PASJ 70 (2018) S25 1705.06745.

[89] A. H. Wright, H. Hildebrandt, K. Kuijken, T. Erben, R. Blake, H. Buddelmeijer et al., KiDS+VIKING-450: 
A new combined optical and near-infrared dataset for cosmology and astrophysics, A\&A 632 (2019) A34 1812.06077 .

[90] C. Laigle, H. J. McCracken, O. Ilbert, B. C. Hsieh, I. Davidzon, P. Capak et al., The COSMOS2015 Catalog: Exploring the $1<z<6$ Universe with Half a Million Galaxies, ApJS 224 (2016) 241604.02350.

[91] M. M. Ivanov, E. McDonough, J. C. Hill, M. Simonović, M. W. Toomey, S. Alexander et al., Constraining Early Dark Energy with Large-Scale Structure, 2006.11235

[92] J. Halverson, C. Long, B. Nelson and G. Salinas, Towards string theory expectations for photon couplings to axionlike particles, Phys. Rev. D100 (2019) 106010 1909.05257.

[93] N. Arkani-Hamed, L. Motl, A. Nicolis and C. Vafa, The String landscape, black holes and gravity as the weakest force, JHEP 06 (2007) 060 hep-th/0601001.

[94] M. Hasselfield, M. Hilton, T. A. Marriage, G. E. Addison, L. F. Barrientos, N. Battaglia et al., The Atacama Cosmology Telescope: Sunyaev-Zel'dovich selected galaxy clusters at $148 \mathrm{GHz}$ from three seasons of data, JCAP 2013 (2013) 008 1301.0816.

[95] A. B. Mantz, A. von der Linden, S. W. Allen, D. E. Applegate, P. L. Kelly, R. G. Morris et al., Weighing the giants - IV. Cosmology and neutrino mass, MNRAS 446 (2015) 22051407.4516.

[96] Planck Collaboration, P. A. R. Ade, N. Aghanim, M. Arnaud, M. Ashdown, J. Aumont et al., Planck 2015 results. XXIV. Cosmology from Sunyaev-Zeldovich cluster counts, A\&A 594 (2016) A24 1502.01597.

[97] S. Bocquet, J. P. Dietrich, T. Schrabback, L. E. Bleem, M. Klein, S. W. Allen et al., Cluster Cosmology Constraints from the $2500 \mathrm{deg}^{2}$ SPT-SZ Survey: Inclusion of Weak Gravitational Lensing Data from Magellan and the Hubble Space Telescope, ApJ 878 (2019) 551812.01679.

[98] Planck Collaboration, N. Aghanim, M. Arnaud, M. Ashdown, J. Aumont, C. Baccigalupi et al., Planck 2015 results. XXII. A map of the thermal Sunyaev-Zeldovich effect, A\&A 594 (2016) A22 1502.01596 .
[99] B. Bolliet, B. Comis, E. Komatsu and J. F. Macías-Pérez, Dark energy constraints from the thermal Sunyaev-Zeldovich power spectrum, MNRAS 477 (2018) 4957 1712.00788.

[100] J. Tinker, A. V. Kravtsov, A. Klypin, K. Abazajian, M. Warren, G. Yepes et al., Toward a Halo Mass Function for Precision Cosmology: The Limits of Universality, ApJ 688 (2008) 709 0803.2706.

[101] D. Grin, M. A. Amin, V. Gluscevic, R. Hlǒzek, D. J. E. Marsh, V. Poulin et al., Gravitational probes of ultra-light axions, 1904.09003.

[102] R. Hlozek, D. Grin, D. J. E. Marsh and P. G. Ferreira, A search for ultralight axions using precision cosmological data, Phys. Rev. D91 (2015) 103512 1410.2896.

[103] R. Hložek, D. J. E. Marsh, D. Grin, R. Allison, J. Dunkley and E. Calabrese, Future CMB tests of dark matter: Ultralight axions and massive neutrinos, Phys. Rev. D95 (2017) 123511 1607.08208.

[104] L. M. Capparelli, R. R. Caldwell and A. Melchiorri, Cosmic Birefringence Test of the Hubble Tension, 1909.04621

[105] S. Alexander, E. McDonough, A. Pullen and B. Shapiro, Physics Beyond The Standard Model with Circular Polarization in the $C M B$ and $C M B-21 \mathrm{~cm}$ Cross-Correlation, JCAP 2001 (2020) 032 1911.01418 .

[106] I. L. Padilla et al., Two-year Cosmology Large Angular Scale Surveyor (CLASS) Observations: A Measurement of Circular Polarization at $40 \mathrm{GHz}$, 1911.00391

[107] J. Sakstein, H. Desmond and B. Jain, Screened Fifth Forces Mediated by Dark Matter-Baryon Interactions: Theory and Astrophysical Probes, Phys. Rev. D100 (2019) 1040351907.03775 .

[108] H. Desmond, B. Jain and J. Sakstein, Local resolution of the Hubble tension: The impact of screened fifth forces on the cosmic distance ladder, Phys. Rev. D100 (2019) 043537 1907.03778.

[109] H. Desmond and J. Sakstein, Screened fifth forces lower the TRGB-calibrated Hubble constant too, 2003.12876. 
Appendix A: Constraints on EDE scalar field decay constant and mass

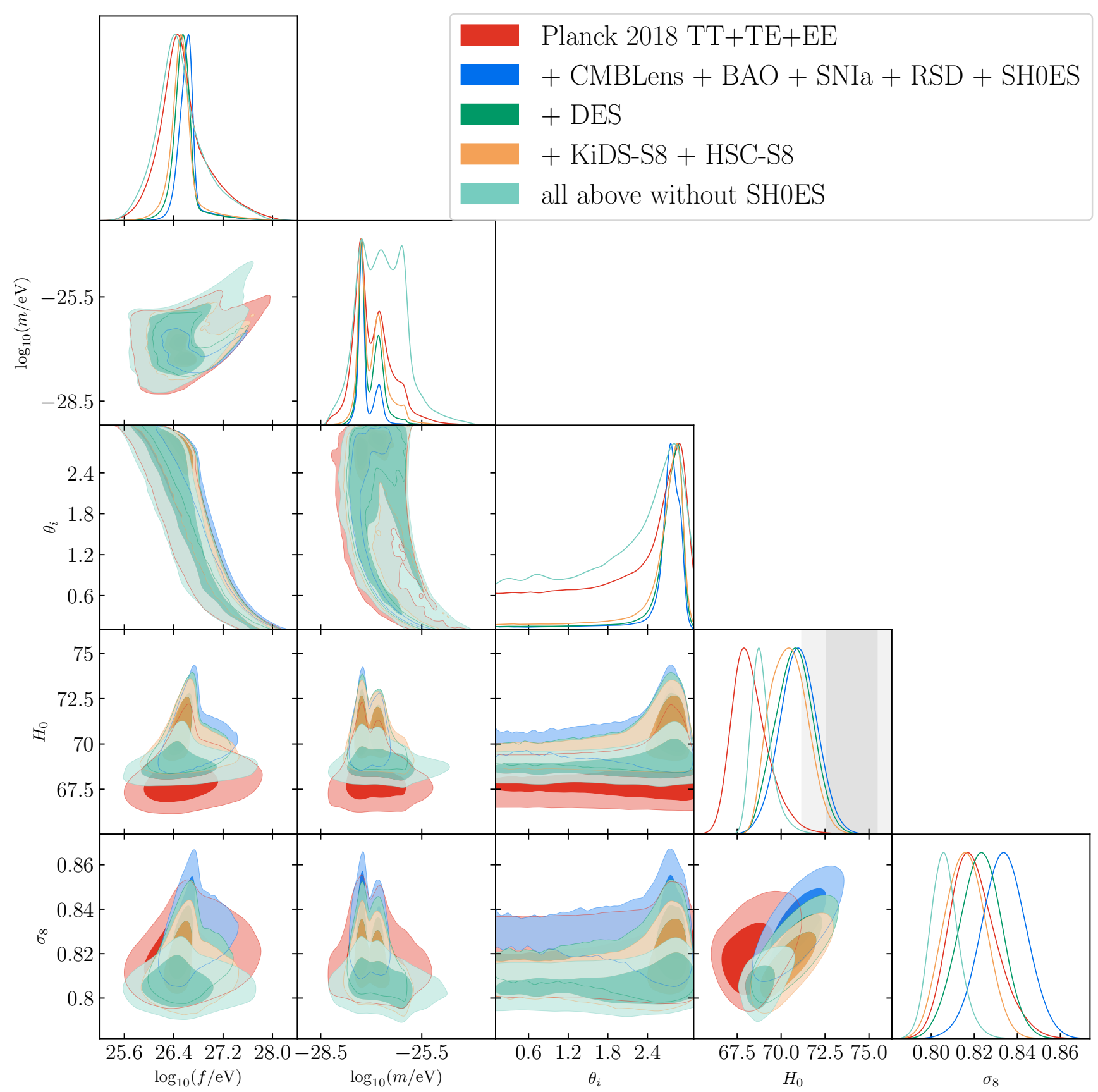

FIG. 16: Constraints on the EDE scenario from a variety of cosmological data sets. The analyses shown are identical to those in Fig. 1, but with the fundamental physics parameters $\log _{10}(f)$ and $\log _{10}(m)$ displayed instead of the effective EDE parameters $f_{\mathrm{EDE}}$ and $\log _{10}\left(z_{c}\right)$. 


\section{Appendix B: Validation of $S_{8}$ prior approach}

In this appendix, we test the approximation of replacing the full DES 3x2pt likelihood with a Gaussian prior on $S_{8}$ corresponding to the DES measurement. While the DES data most tightly constrain $S_{8}$, they also yield a reasonably strong constraint on $\Omega_{m}$ 10. It is thus not a priori clear that a simple $S_{8}$ prior is an adequate substitute for the full likelihood. However, as we will see, since DES only weakly constrains any non- $S_{8}$ parameter in comparison to Planck (including $\Omega_{m}$ ), this approximation turns out to be excellent when performing a joint analysis of these data sets.

We are particularly interested in the validity of this approach in the context of the EDE model, and in the context of the data set combination utilized here. Thus, we consider the posterior distributions obtained for the full DES likelihood and for a DES prior on $S_{8}$. The results for the EDE model are shown in Figs. 17 and 18 The figures display a near-perfect match of the posteriors, thus demonstrating that the $S_{8}$ prior is an excellent approximation to the full DES likelihood in the EDE model, within the context of this data set combination. For completeness, we perform the same comparison for $\Lambda \mathrm{CDM}$, shown in Fig. 19. The match is again excellent, although we note a slight $(\ll 1 \sigma)$ shift in the peak of the $H_{0}$ posterior. This is caused by the $\Omega_{m}-H_{0}$ degeneracy in $\Lambda$ CDM, and the fact that the $S_{8}$ prior approach does not include the DES constraining power on $\Omega_{m}$.

From these comparisons we conclude that the $S_{8}$ prior is an excellent approximation to the full DES 3x2pt likelihood in the EDE and $\Lambda$ CDM models. However, as evidenced by the small yet visible shift of the $H_{0}$ posterior in $\Lambda$ CDM, this claim should be evaluated on a model-by-model basis.

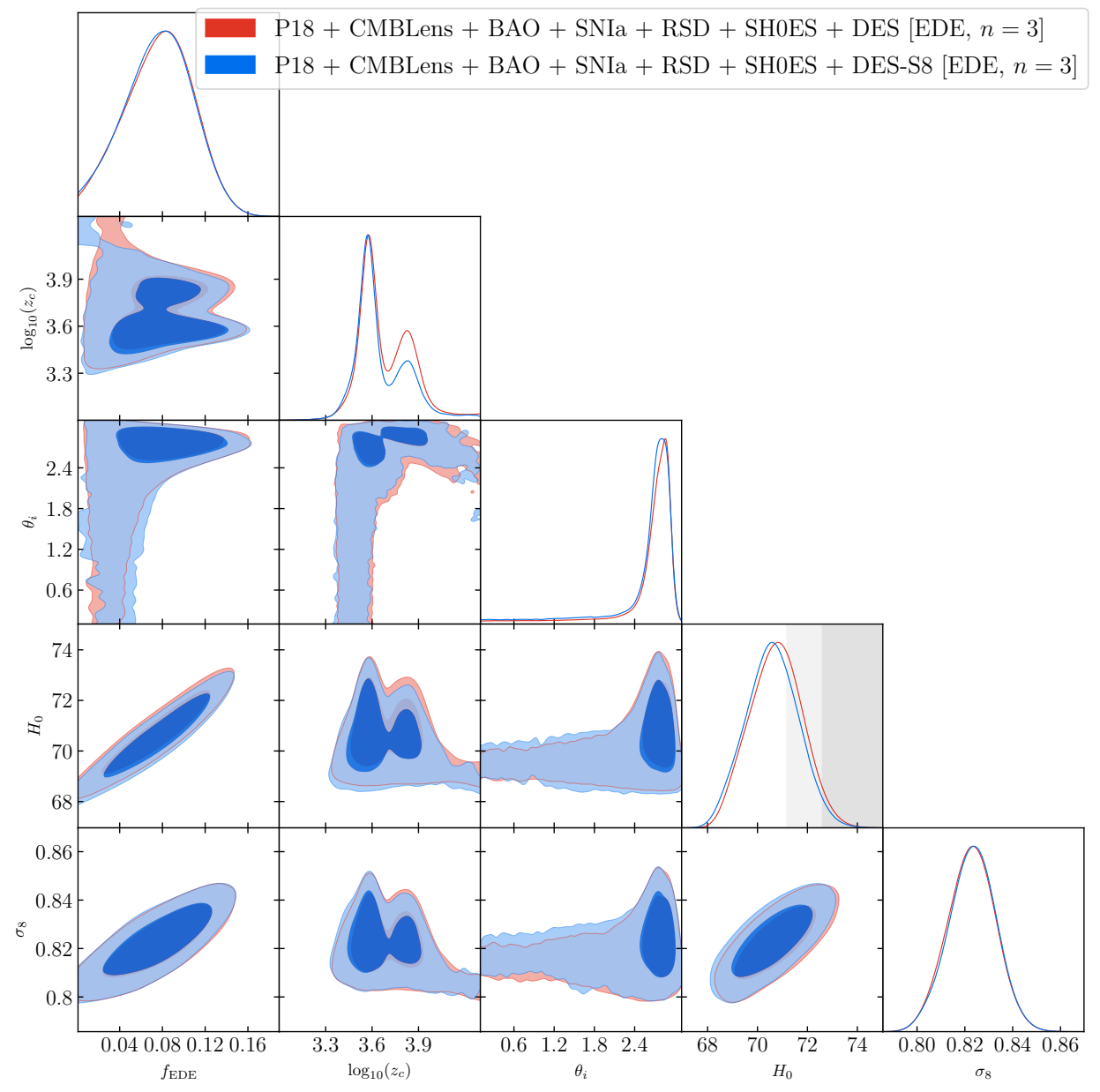

FIG. 17: Validation of an $S_{8}$ prior as a good approximation to the inclusion of the full DES-Y1 3x2pt likelihood in the EDE model. We compare the posterior distributions in the fit of EDE to Planck 2018 primary CMB data (TT+TE+EE); Planck 2018 CMB lensing data; BAO data from 6dF, SDSS DR7, and SDSS DR12; Pantheon SNIa data; the latest SH0ES $H_{0}$ constraint; SDSS DR12 RSD data; and the DES-Y1 3x2pt data (red), to the posterior distributions in the fit to same data set but with DES-Y1 3x2pt data replaced by a prior on $S_{8}$ (blue), $S_{8}=0.773_{-0.020}^{+0.026}$. The close agreement of the posteriors indicates a Gaussian prior on $S_{8}$ is an excellent approximation to the inclusion of the full DES-Y1 3x2pt likelihood in this data combination in the context of EDE. 


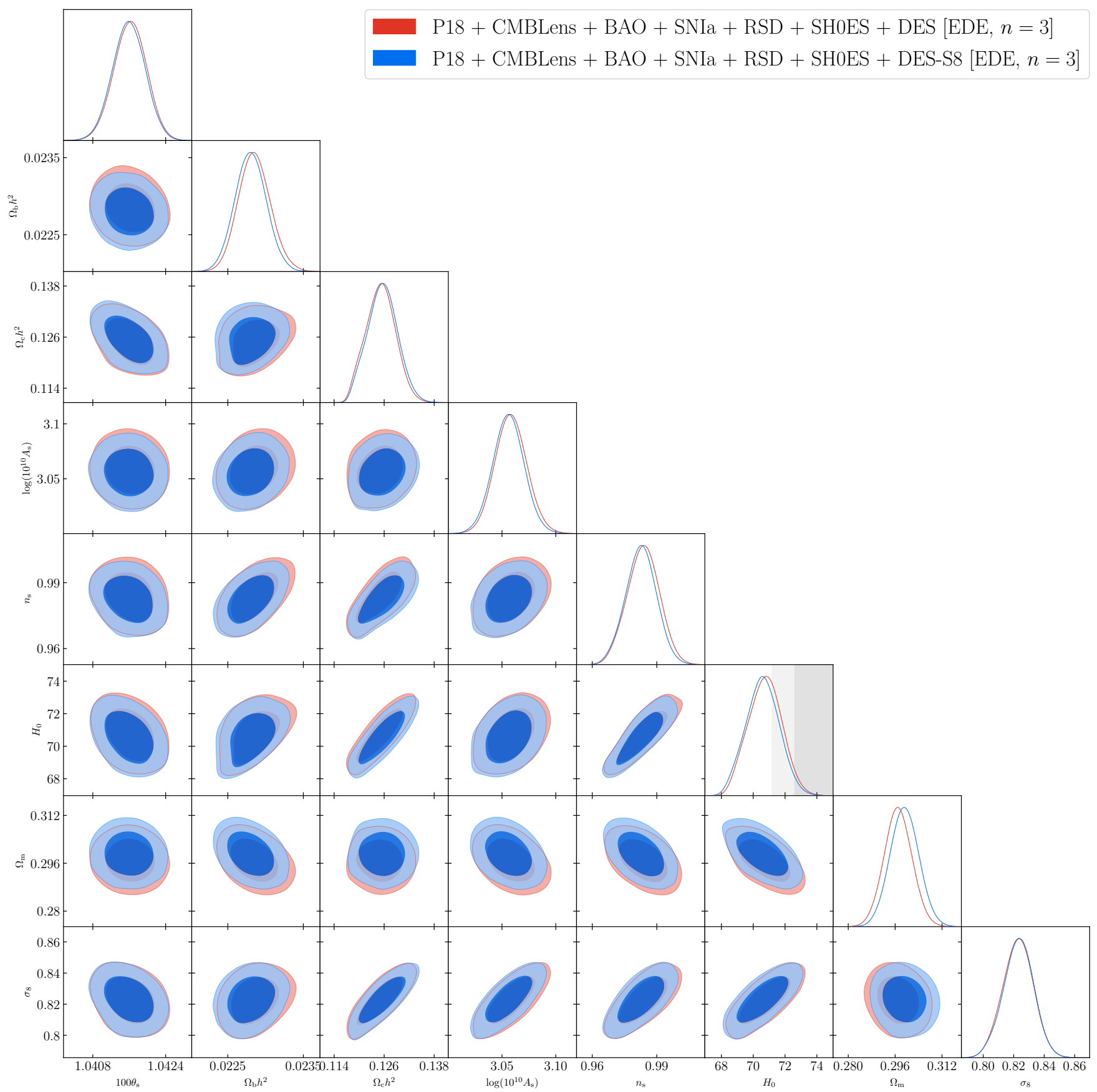

FIG. 18: Validation of an $S_{8}$ prior as a good approximation to the inclusion of the full DES likelihood in the EDE model, analogous to Fig. 17 but here showing the standard cosmological parameters. We compare the posterior distributions in the fit of EDE to Planck 2018 primary CMB data (TT+TE+EE); Planck 2018 CMB lensing data; BAO data from 6dF, SDSS DR7, and SDSS DR12; Pantheon SNIa data; the latest SH0ES $H_{0}$ constraint; SDSS DR12 RSD data; and the DES-Y1 3x2pt data (red), to the posterior distributions in the fit to same data set but with DES $3 \times 2$ pt data replaced by a prior on $S_{8}$ (blue), $S_{8}=0.773_{-0.020}^{+0.026}$. The close agreement of the posteriors indicates a Gaussian prior on $S_{8}$ is an excellent approximation to the inclusion of the DES $3 \times 2$ pt likelihood in this data combination in the context of EDE. 


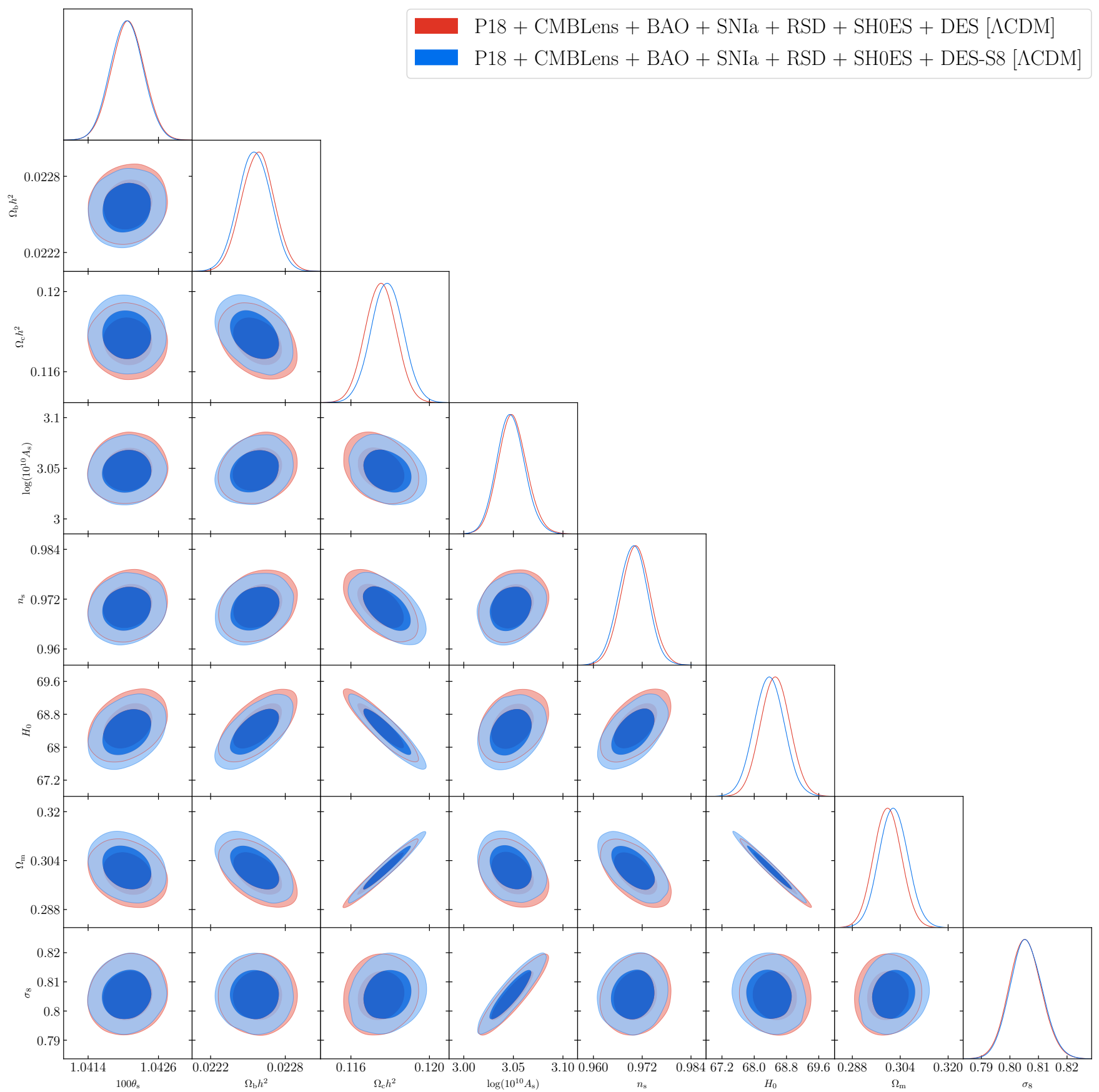

FIG. 19: Validation of an $S_{8}$ prior as a good approximation to the inclusion of the full DES likelihood in $\Lambda$ CDM. We compare the posterior distributions in the fit of $\Lambda$ CDM to Planck 2018 primary CMB data (TT+TE+EE); Planck 2018 CMB lensing data; BAO data from 6dF, SDSS DR7, and SDSS DR12; Pantheon SNIa data; the latest SH0ES $H_{0}$ constraint; SDSS DR12 RSD data; and the DES-Y1 3x2pt data (red), to the posterior distributions in the fit to same data set but with DES 3x2pt data replaced by a prior on $S_{8}$ (blue), $S_{8}=0.773_{-0.020}^{+0.026}$. The close agreement of the posteriors indicates a Gaussian prior on $S_{8}$ is an excellent approximation to the inclusion of the DES 3x2pt likelihood in this data set combination in the context of $\Lambda$ CDM. The slight $(\ll 1 \sigma)$ shift in $H_{0}$ arises due to the constraining power of DES on $\Omega_{m}$, which is not captured in the simple $S_{8}$ prior approach, and the tight correlation between $\Omega_{m}$ and $H_{0}$ in $\Lambda$ CDM. 


\section{Appendix C: Restriction to Sub-Planckian Axion Decay Constants}

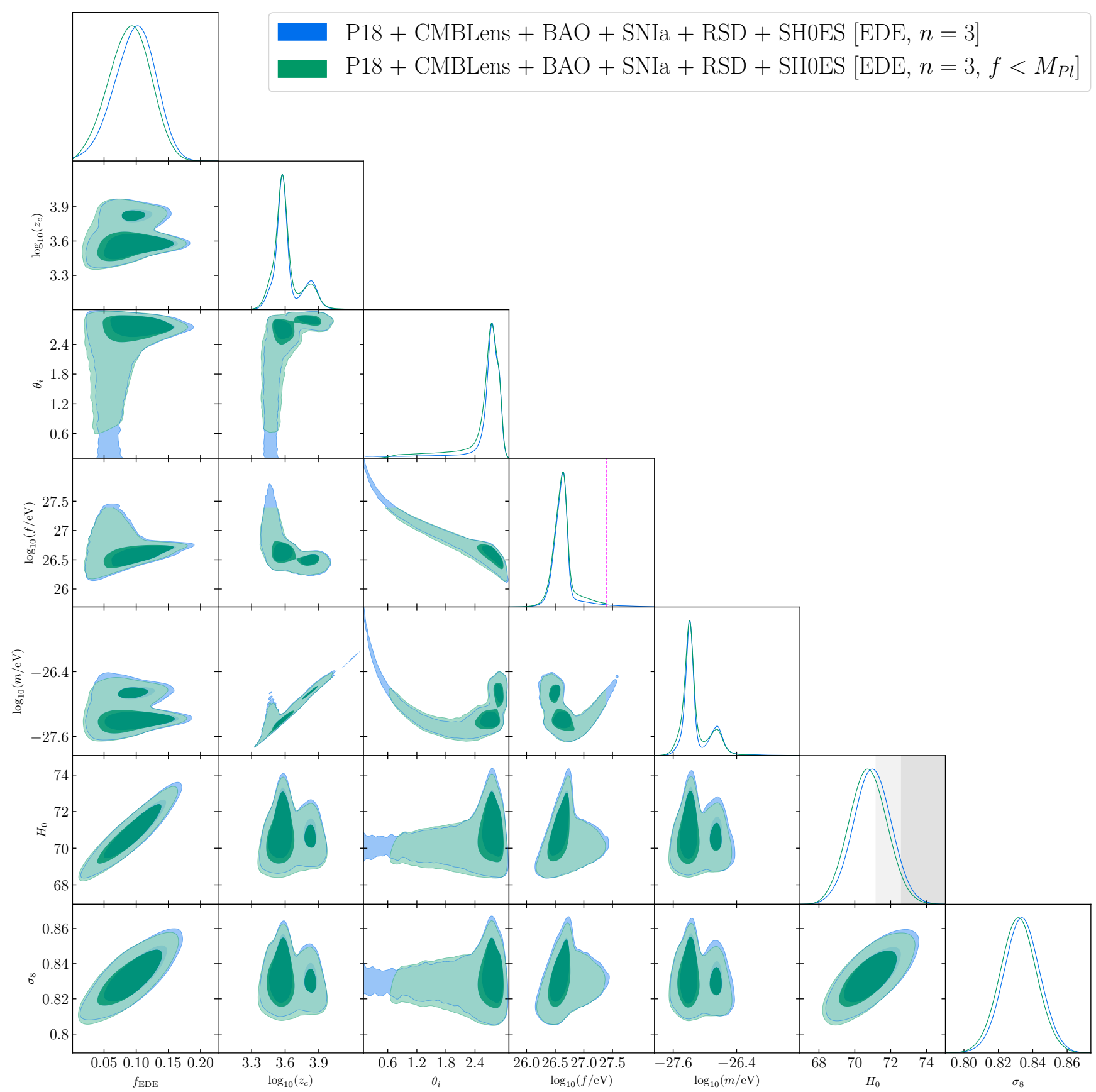

FIG. 20: Cosmological parameter constraints with an upper bound imposed on the axion decay constant, $f \leq M_{p l}$. See Sec. VII 


\section{Appendix D: Additional Figures Comparing EDE and $\Lambda$ CDM Predictions}

In what follows we include additional figures displaying the difference between the best-fit EDE and $\Lambda$ CDM models to non-LSS data, i.e., CMB, SH0ES, and distance data (RSD data were also included in the fits, but play very little role due to their relatively large error bars). The parameters used correspond to the best-fit values from Table 1 of [26. For EDE, these are given in Eq. (7),

$$
\begin{gathered}
H_{0}=72.19 \mathrm{~km} / \mathrm{s} / \mathrm{Mpc}, 100 \omega_{b}=2.253, \omega_{\mathrm{cdm}}=0.1306,10^{9} A_{s}=2.215, n_{s}=0.9889, \tau_{\text {reio }}=0.072 \\
f_{\mathrm{EDE}}=0.122, \log _{10}\left(z_{c}\right)=3.562, \theta_{i}=2.83 .
\end{gathered}
$$

while for $\Lambda \mathrm{CDM}$, these are given in Eq. 10,

$$
H_{0}=68.21 \mathrm{~km} / \mathrm{s} / \mathrm{Mpc}, 100 \omega_{b}=2.253, \omega_{\mathrm{cdm}}=0.1177,10^{9} A_{s}=2.216, n_{s}=0.9686 \quad \tau_{\text {reio }}=0.085 .
$$

We show the CMB lensing convergence auto-power spectrum, $C_{L}^{\kappa \kappa}$, and the fractional difference between EDE and $\Lambda \mathrm{CDM}$ in Fig. 21. The impact here is substantial at high $L$, giving rise to shifts from $\Lambda \mathrm{CDM}$ of $\mathcal{O}(10 \%)$, consistent with the changes induced in the matter power spectrum (see Sec. IV). It should be noted that the changes in the CMB lensing and matter power spectra are driven by the sizeable shift in the physical CDM density $\omega_{\text {cdm }}$, as well as the shift in the scalar spectral index $n_{s}$; these shifts are also what preserve the fit to the primary CMB power spectra. Fig. 22 shows the primary CMB power spectra $D_{\ell}^{E E}$ (left) and $D_{\ell}^{T E}$ (right) and the fractional difference between the two models. These results further emphasize the remarkable agreement between the two models in the CMB, as was displayed in Fig. 3 for the temperature power spectrum. We additionally include the fractional difference for $f \sigma_{8}$ and $\sigma_{8}$ in Fig. 23. We conclude with a comparison of the growth factors and their fractional differences for both cosmologies in Fig. 24 which illustrate the effects of the EDE on the growth of perturbations over nearly all of cosmic history.
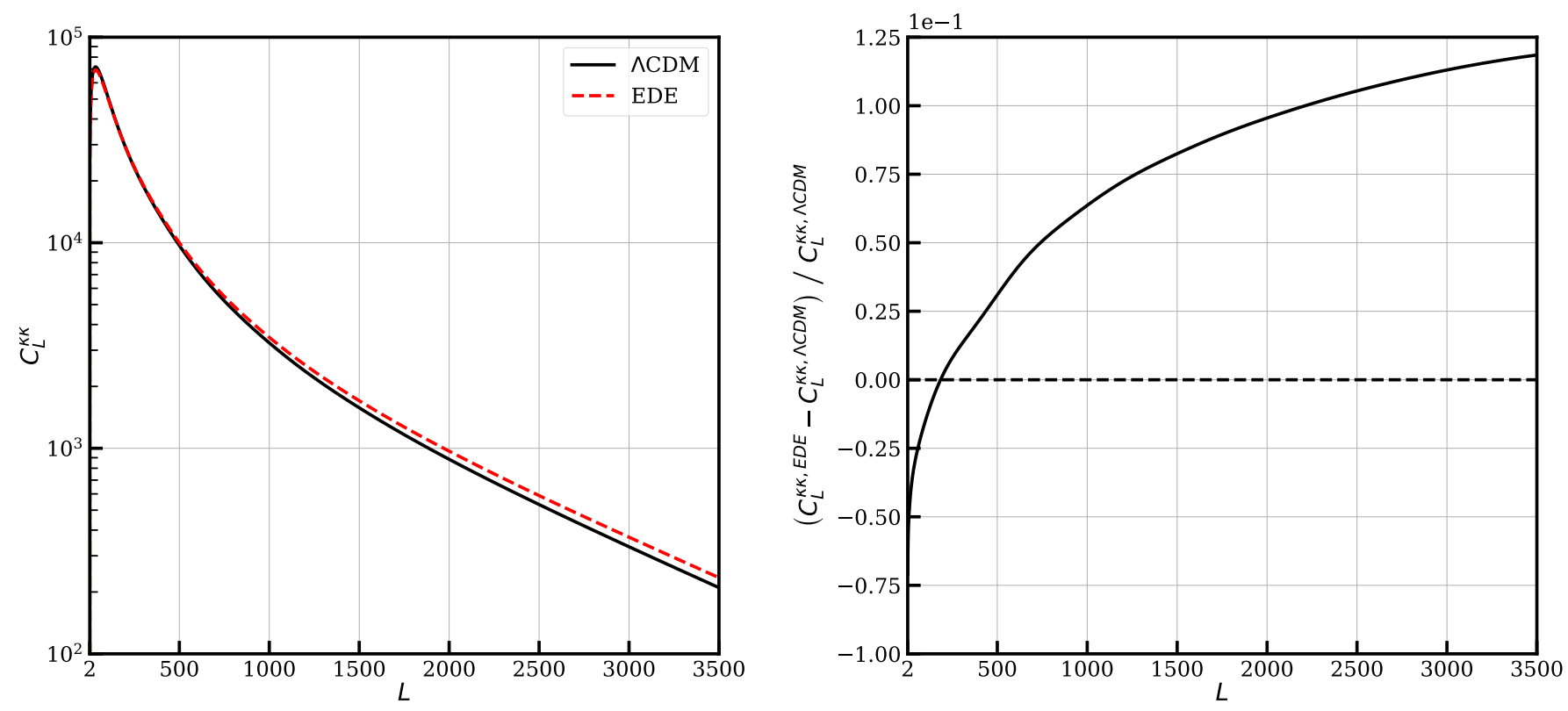

FIG. 21: CMB lensing convergence auto-power spectrum in EDE with parameters given in Eq. (7) and $\Lambda$ CDM with parameters given in Eq. 10, and the fractional difference between them. The standard $\Lambda$ CDM parameters differ non-negligibly between the models, and similar to the matter power spectrum observed in Sec. IV this generates a substantial change in the CMB lensing power spectrum. The change is $\mathcal{O}(10 \%)$ at high- $L$, driven primarily by the shift in $\Lambda$ CDM parameters, and not effects of the EDE itself. 

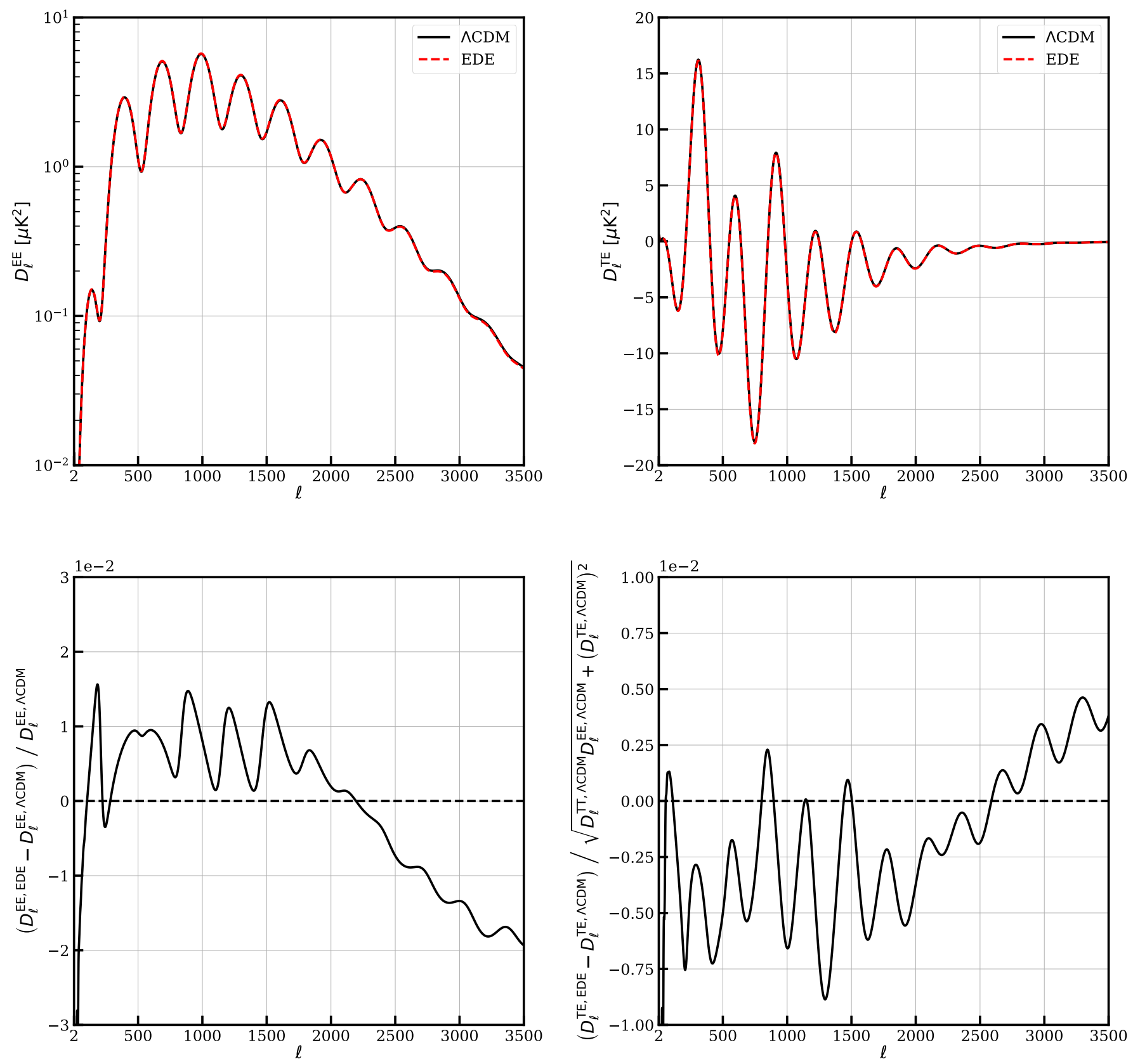

FIG. 22: CMB EE (left) and TE (right) power spectra in EDE with parameters given in Eq. (7) and $\Lambda$ CDM with parameters given in Eq. 10, and fractional difference between EDE and $\Lambda$ CDM (bottom). The parameters for both models correspond to the best-fit values from Table 1 of 26. The standard $\Lambda$ CDM parameters differ non-negligibly between the models, while producing remarkably similar CMB temperature and polarization power spectra. Note that in the fractional difference plot for TE we have normalized by the variance, differing in our convention relative to other figures, because of the zero crossings of the TE cross-correlation. 

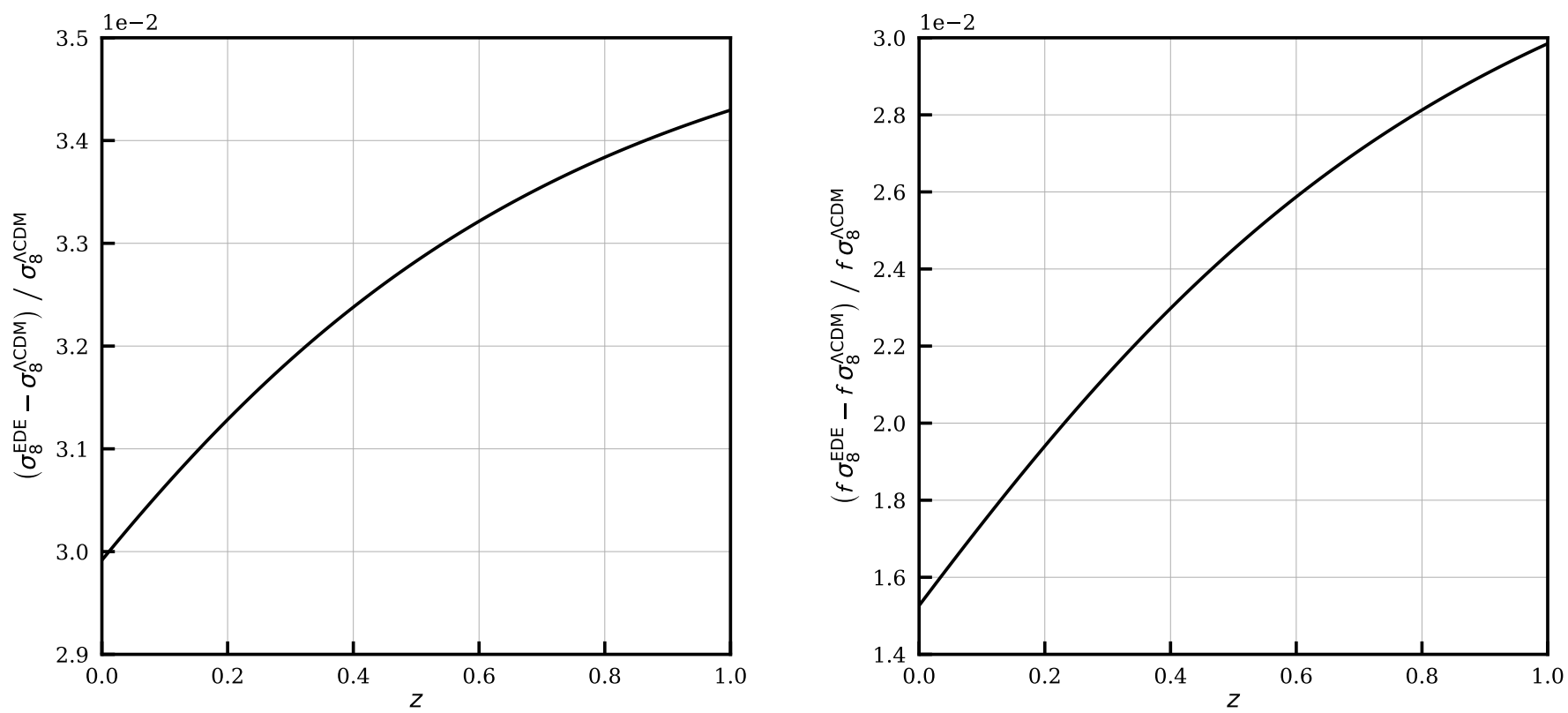

FIG. 23: Fractional difference of $\sigma_{8}(z)$ and $f \sigma_{8}(z)$ in EDE with parameters given in Eq. (7) and $\Lambda$ CDM with parameters given in Eq. 10]. The parameters for both models correspond to the best-fit values from Table 1 of [26]. The standard $\Lambda \mathrm{CDM}$ parameters differ non-negligibly between the models, leading to the changes seen here, while producing remarkably close CMB power spectra (see Figs. 3 and 22 .
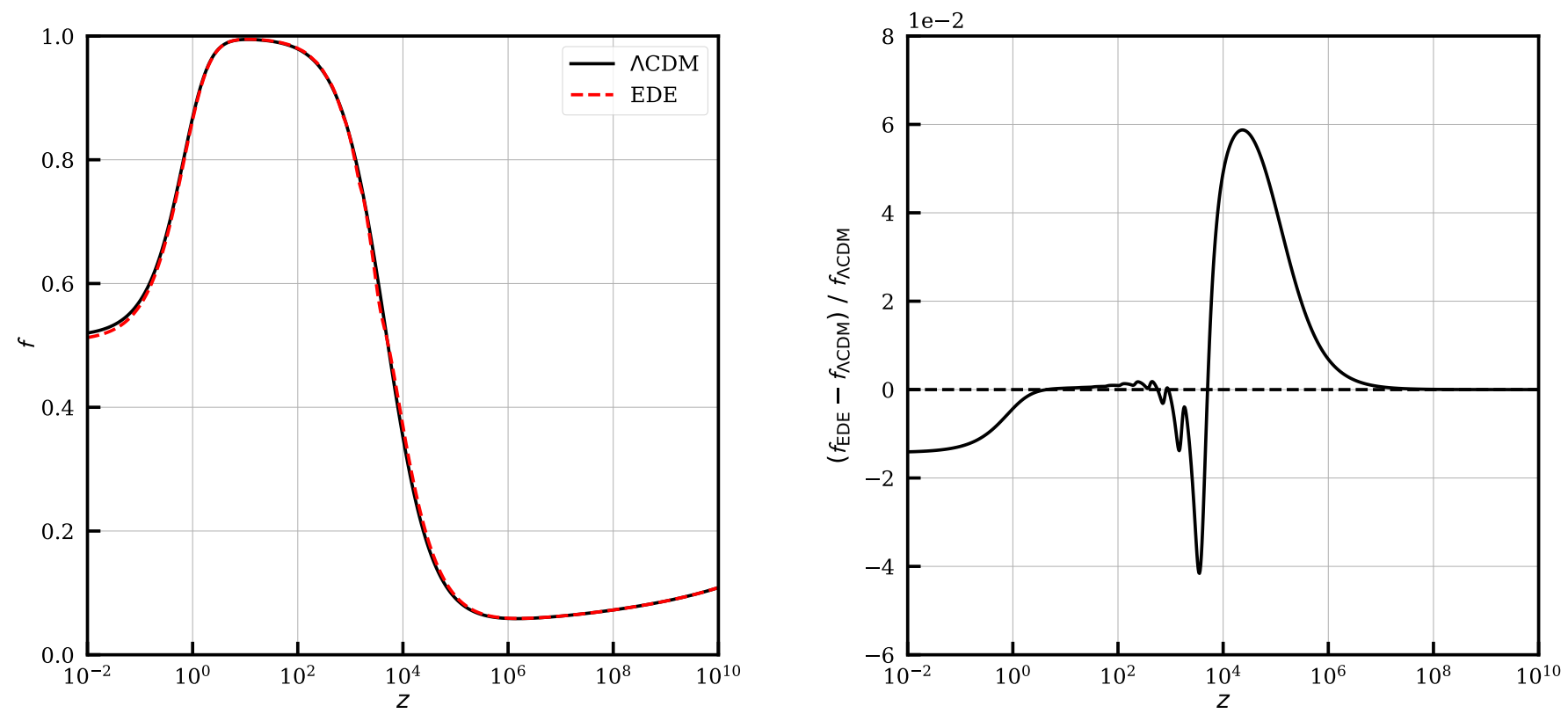

FIG. 24: Growth factor $f$ in EDE vs. $\Lambda$ CDM (left) and fractional difference between the two (right). The models and parameters are identical to those used in Fig. 23 (and elsewhere in this appendix). Note that this plot covers a very wide range in redshift, in order to show the impact of the EDE field on the growth of perturbations over all of cosmic history. 
Appendix E: Parameter Constraints from Credible-Interval Approach

Constraints from Planck 2018 data only: TT+TE+EE

\begin{tabular}{|c|c|c|c|c|}
\hline Parameter & $\Lambda$ CDM Best-Fit & $\bar{~} \Lambda$ CDM Marg. & EDE $(n=3)$ Best-Fit & EDE $(n=3)$ Marg. \\
\hline $\ln \left(10^{10} A_{s}\right)$ & 3.055 & $3.044 \pm 0.016$ & 3.056 & $3.051 \pm 0.017$ \\
\hline$n_{\mathrm{s}}$ & 0.9659 & $0.9645 \pm 0.0043$ & 0.9769 & $0.9702_{-0.0083}^{+0.0053}$ \\
\hline $100 \theta_{\mathrm{s}}$ & 1.04200 & $1.04185 \pm 0.00029$ & 1.04168 & $1.04164 \pm 0.00034$ \\
\hline$\Omega_{\mathrm{b}} h^{2}$ & 0.02244 & $0.02235 \pm 0.00015$ & 0.02250 & $0.02250_{-0.00022}^{+0.00018}$ \\
\hline$\Omega_{\mathrm{c}} h^{2}$ & 0.1201 & $0.1202 \pm 0.0013$ & 0.1268 & $0.1234_{-0.0038}^{+0.0019}$ \\
\hline$\tau_{\text {reio }}$ & 0.0587 & $0.0541 \pm 0.0076$ & 0.0539 & $0.0549 \pm 0.0078$ \\
\hline $\log _{10}\left(z_{c}\right)$ & - & - & 3.75 & $3.66_{-0.28}^{+0.24}$ \\
\hline$f_{\text {EDE }}$ & - & - & 0.068 & $<0.087$ \\
\hline$\theta_{i}$ & - & - & 2.96 & $>0.36$ \\
\hline$H_{0}[\mathrm{~km} / \mathrm{s} / \mathrm{Mpc}]$ & 67.44 & $67.29 \pm 0.59$ & 69.13 & $68.29_{-1.2}^{+0.73}$ \\
\hline$\Omega_{\mathrm{m}}$ & 0.3147 & $0.3162 \pm 0.0083$ & 0.3138 & $0.3145 \pm 0.0086$ \\
\hline$\sigma_{8}$ & 0.8156 & $0.8114 \pm 0.0073$ & 0.8280 & $0.8198_{-0.012}^{+0.0090}$ \\
\hline$S_{8}$ & 0.8355 & $0.8331 \pm 0.0159$ & 0.8468 & $0.8393 \pm 0.0175$ \\
\hline $\log _{10}(f / e V)$ & - & - & 26.36 & $26.57_{-0.46}^{+0.26}$ \\
\hline $\log _{10}(m / e V)$ & - & - & -26.90 & $-26.94_{-0.65}^{+0.39}$ \\
\hline
\end{tabular}

TABLE XI: The best-fit and mean $\pm 1 \sigma$ constraints on the cosmological parameters in $\Lambda$ CDM and in the EDE scenario with $n=3$, as inferred from Planck 2018 primary CMB data only (TT+TE+EE). Upper and lower limits are quoted at 95\% CL. The EDE component is consistent with zero. These constraints are computed with the credible-interval approach discussed near the beginning of Sec. VI and can be compared with the equal-tail limits presented in Table II] (the best-fit and mean values are, of course, identical in the two tables).

Constraints from Planck 2018 TT+TE+EE + CMB Lensing, BAO, SNIa, SH0ES, and RSD

\begin{tabular}{|l|c|c|c|c|}
\hline \hline Parameter & $\Lambda$ CDM Best-Fit & $\Lambda$ CDM Marg. & EDE $(n=3)$ Best-Fit & EDE $(n=3)$ Marg. \\
\hline \hline $\boldsymbol{l n}\left(\mathbf{1 0}^{\mathbf{1 0}} \boldsymbol{A}_{\mathbf{s}}\right)$ & 3.047 & $3.051 \pm 0.014$ & 3.058 & $3.064 \pm 0.015$ \\
$\boldsymbol{n}_{\mathbf{s}}$ & 0.9686 & $0.9689 \pm 0.0036$ & 0.9847 & $0.9854 \pm 0.0070$ \\
$\mathbf{1 0 0}_{\mathbf{s}}$ & 1.04209 & $1.04204 \pm 0.00028$ & 1.04119 & $1.04144 \pm 0.00037$ \\
$\boldsymbol{\Omega}_{\mathbf{b}} \boldsymbol{h}^{\mathbf{2}}$ & 0.02249 & $0.02252 \pm 0.00013$ & 0.02286 & $0.02280 \pm 0.00021$ \\
$\boldsymbol{\Omega}_{\mathbf{c}} \boldsymbol{h}^{\mathbf{2}}$ & 0.11855 & $0.11830 \pm 0.00086$ & 0.12999 & $0.1290 \pm 0.0039$ \\
$\boldsymbol{\tau}_{\mathbf{r e i o}}$ & 0.0566 & $0.0590_{-0.0076}^{+0.0067}$ & 0.0511 & $0.0573 \pm 0.0073$ \\
$\log _{\mathbf{1 0}}\left(\boldsymbol{z}_{\boldsymbol{c}}\right)$ & - & - & 3.59 & $3.63_{-0.14}^{+0.22}$ \\
$\boldsymbol{f}_{\mathbf{E D E}}$ & - & - & 0.105 & $0.098_{-0.029}^{+0.034}$ \\
$\boldsymbol{\theta}_{\boldsymbol{i}}$ & - & - & 2.71 & $2.58_{+0.05}^{+0.35}$ \\
\hline$H_{0}[\mathrm{~km} / \mathrm{s} / \mathrm{Mpc}]$ & 68.07 & $68.17 \pm 0.39$ & 71.15 & $71.0 \pm 1.1$ \\
$\Omega_{\mathrm{m}}$ & 0.3058 & $0.3044 \pm 0.0051$ & 0.3032 & $0.3025 \pm 0.0051$ \\
$\sigma_{8}$ & 0.8081 & $0.8086 \pm 0.0060$ & 0.8322 & $0.834 \pm 0.011$ \\
$S_{8}$ & 0.8158 & $0.8145 \pm 0.0099$ & 0.8366 & $0.837 \pm 0.013$ \\
$\log _{10}(f / \mathrm{eV})$ & - & - & 26.63 & $26.64_{-0.15}^{+0.08}$ \\
$\log _{10}(\mathrm{~m} / \mathrm{eV})$ & - & - & -27.27 & $-27.15_{-0.29}^{+0.41}$ \\
\hline
\end{tabular}

TABLE XII: The best-fit and mean $\pm 1 \sigma$ constraints on the cosmological parameters in $\Lambda$ CDM and in the EDE scenario with $n=3$, as inferred from the combination of Planck 2018 primary CMB data (TT+TE+EE); Planck 2018 CMB lensing data; BAO data from 6dF, SDSS DR7, and SDSS DR12; Pantheon SNIa data; the latest SH0ES $H_{0}$ constraint; and SDSS DR12 RSD data. The EDE component is detected at $3.4 \sigma$ significance (using the credible-interval-derived error bar here). These constraints are computed with the credible-interval approach discussed near the beginning of Sec. VI] and can be compared with the equal-tail limits presented in Table IV (the best-fit and mean values are, of course, identical in the two tables). 
Constraints from Planck 2018 TT+TE+EE + CMB Lensing, BAO, SNIa, SH0ES, RSD, and DES-Y1

\begin{tabular}{|c|c|c|c|c|}
\hline Parameter & $\Lambda$ CDM Best-Fit & $\Lambda$ CDM Marg. & EDE $(n=3)$ Best-Fit & EDE $(n=3)$ Marg. \\
\hline $\ln \left(10^{10} A_{s}\right)$ & 3.049 & $3.049 \pm 0.014$ & 3.064 & $3.058 \pm 0.015$ \\
\hline$n_{\mathrm{s}}$ & 0.9698 & $0.9704 \pm 0.0035$ & 0.9909 & $0.9838 \pm 0.0074$ \\
\hline $100 \theta_{\mathrm{s}}$ & 1.04183 & $1.04208 \pm 0.00028$ & 1.04172 & $1.04162 \pm 0.00036$ \\
\hline$\Omega_{\mathrm{b}} h^{2}$ & 0.02260 & $0.02258 \pm 0.00013$ & 0.02304 & $0.02285 \pm 0.00021$ \\
\hline$\Omega_{\mathrm{c}} h^{2}$ & 0.11810 & $0.11752 \pm 0.00078$ & 0.1254 & $0.1251 \pm 0.0035$ \\
\hline$\tau_{\text {reio }}$ & 0.0584 & $0.0590_{-0.0076}^{+0.0067}$ & 0.0626 & $0.0581 \pm 0.0074$ \\
\hline $\log _{10}\left(z_{c}\right)$ & - & - & 3.84 & $3.69 \pm 0.18$ \\
\hline$f_{\mathrm{EDE}}$ & - & - & 0.088 & $0.077_{-0.030}^{+0.035}$ \\
\hline$\theta_{i}$ & - & - & 2.89 & $2.58_{+0.035}^{+0.38}$ \\
\hline$H_{0}[\mathrm{~km} / \mathrm{s} / \mathrm{Mpc}]$ & 68.24 & $68.52 \pm 0.36$ & 71.05 & $70.7 \pm 1.0$ \\
\hline$\Omega_{\mathrm{m}}$ & 0.3035 & $0.2998 \pm 0.0046$ & 0.2954 & $0.2970 \pm 0.0048$ \\
\hline$\sigma_{8}$ & 0.8067 & $0.8054 \pm 0.0057$ & 0.8263 & $0.823 \pm 0.010$ \\
\hline$S_{8}$ & 0.8115 & $0.8051 \pm 0.0087$ & 0.8199 & $0.819 \pm 0.011$ \\
\hline $\log _{10}(f / e V)$ & - & - & 26.47 & $26.57_{-0.16}^{+0.11}$ \\
\hline $\log _{10}(m / e V)$ & - & - & -26.76 & $-27.03 \pm 0.38$ \\
\hline
\end{tabular}

TABLE XIII: The best-fit and mean $\pm 1 \sigma$ constraints on the cosmological parameters in $\Lambda$ CDM and in the EDE scenario with $n=3$, as inferred from the combination of Planck 2018 primary CMB data (TT+TE+EE); Planck 2018 CMB lensing data; BAO data from 6dF, SDSS DR7, and SDSS DR12; Pantheon SNIa data; the latest SH0ES $H_{0}$ constraint; SDSS DR12 RSD data; and DES-Y1 3x2pt data. The inclusion of the DES data decreases the evidence for EDE to 2.6 $\sigma$ significance (using the credible-interval-derived error bar here). These constraints are computed with the credible-interval approach discussed near the beginning of Sec. VI and can be compared with the equal-tail limits presented in Table VI (the best-fit and mean values are, of course, identical in the two tables).

Constraints from Planck 2018 TT+TE+EE + CMB Lensing, BAO, SNIa, SH0ES, RSD, DES-Y1, KiDS- $S_{8}$, and HSC- $S_{8}$

\begin{tabular}{|c|c|c|}
\hline Parameter & $\Lambda$ CDM Marg. & EDE $(n=3)$ Marg. \\
\hline $\ln \left(10^{10} A_{\mathrm{s}}\right)$ & $3.046 \pm 0.014$ & $3.053 \pm 0.015$ \\
\hline$n_{\mathrm{s}}$ & $0.9710 \pm 0.0035$ & $0.9813 \pm 0.0074$ \\
\hline $100 \theta_{\mathrm{s}}$ & $1.04209 \pm 0.00028$ & $1.04169_{-0.00034}^{+0.00037}$ \\
\hline$\Omega_{\mathrm{b}} h^{2}$ & $0.02260 \pm 0.00013$ & $0.02285_{-0.00022}^{+0.00019}$ \\
\hline$\Omega_{\mathrm{c}} h^{2}$ & $0.11718 \pm 0.00076$ & $0.1230_{-0.0039}^{+0.0029}$ \\
\hline$\tau_{\text {reio }}$ & $0.0581 \pm 0.0072$ & $0.0574 \pm 0.0073$ \\
\hline $\log _{10}\left(z_{c}\right)$ & - & $3.73_{-0.23}^{+0.18}$ \\
\hline$f_{\mathrm{EDE}}$ & - & $0.062 \pm 0.030$ \\
\hline$\theta_{i}$ & - & $2.49_{+0.048}^{+0.50}$ \\
\hline$H_{0}[\mathrm{~km} / \mathrm{s} / \mathrm{Mpc}]$ & $68.67 \pm 0.35$ & $70.45_{-1.2}^{+0.94}$ \\
\hline$\Omega_{\mathrm{m}}$ & $0.2978 \pm 0.0044$ & $0.2952 \pm 0.0046$ \\
\hline$\sigma_{8}$ & $0.8032 \pm 0.0055$ & $0.8157 \pm 0.0094$ \\
\hline$S_{8}$ & $0.8002 \pm 0.0082$ & $0.809 \pm 0.010$ \\
\hline $\log _{10}(f / e V)$ & - & $26.55_{-0.19}^{+0.12}$ \\
\hline $\log _{10}(\mathrm{~m} / \mathrm{eV})$ & - & $-26.94_{-0.50}^{+0.33}$ \\
\hline
\end{tabular}

TABLE XIV: The mean $\pm 1 \sigma$ constraints on the cosmological parameters in $\Lambda$ CDM and in the EDE scenario with $n=3$, as inferred from the combination of Planck 2018 primary CMB data (TT+TE+EE); Planck 2018 CMB lensing data; BAO data from 6dF, SDSS DR7, and SDSS DR12; Pantheon SNIa data; the latest SH0ES $H_{0}$ constraint; SDSS DR12 RSD data; DES-Y1 3x2pt data; and priors on $S_{8}$ derived from KiDS and HSC data. The inclusion of the KiDS and HSC data decreases the evidence for EDE to $2.1 \sigma$ significance (using the credible-interval-derived error bar here). These constraints are computed with the credible-interval approach discussed near the beginning of Sec. VI, and can be compared with the equal-tail limits presented in Table VIII (the mean values are, of course, identical in the two tables). 
Constraints from Planck 2018 TT+TE+EE + CMB Lensing, BAO, SNIa, RSD, DES-Y1, KiDS- $S_{8}$, and HSC-S $($ No-SH0ES)

\begin{tabular}{|c|c|c|}
\hline Parameter & $\Lambda$ CDM Marg. & EDE $(n=3)$ Marg. \\
\hline $\ln \left(10^{10} A_{\mathrm{s}}\right)$ & $3.041 \pm 0.014$ & $3.044 \pm 0.014$ \\
\hline$n_{\mathrm{s}}$ & $0.9691 \pm 0.0035$ & $0.9718_{-0.0055}^{+0.0041}$ \\
\hline $100 \theta_{\mathrm{s}}$ & $1.04200 \pm 0.00028$ & $1.04177_{-0.00030}^{+0.00038}$ \\
\hline$\Omega_{\mathrm{b}} h^{2}$ & $0.02253 \pm 0.00013$ & $0.02264_{-0.00018}^{+0.00015}$ \\
\hline$\Omega_{\mathrm{c}} h^{2}$ & $0.11785 \pm 0.00077$ & $0.11956_{-0.0020}^{+0.00088}$ \\
\hline$\tau_{\text {reio }}$ & $0.0552 \pm 0.0070$ & $0.0558 \pm 0.0070$ \\
\hline $\log _{10}\left(z_{c}\right)$ & - & $>3.28$ \\
\hline$f_{\text {EDE }}$ & - & $<0.060$ \\
\hline$\theta_{i}$ & - & $>0.35$ \\
\hline$H_{0}[\mathrm{~km} / \mathrm{s} / \mathrm{Mpc}]$ & $68.33 \pm 0.36$ & $68.92_{-0.72}^{+0.40}$ \\
\hline$\Omega_{\mathrm{m}}$ & $0.3021 \pm 0.0045$ & $0.3008 \pm 0.0047$ \\
\hline$\sigma_{8}$ & $0.8032 \pm 0.0053$ & $0.8064_{-0.0073}^{+0.0057}$ \\
\hline$S_{8}$ & $0.8060 \pm 0.0082$ & $0.8074 \pm 0.0089$ \\
\hline $\log _{10}(f / e V)$ & - & $26.52_{-0.44}^{+0.28}$ \\
\hline $\log _{10}(\mathrm{~m} / \mathrm{eV})$ & - & $-26.67 \pm 0.69$ \\
\hline
\end{tabular}

TABLE XV: The mean $\pm 1 \sigma$ constraints on the cosmological parameters in $\Lambda$ CDM and in the EDE scenario with $n=3$, as inferred from the combination of Planck 2018 primary CMB data (TT+TE+EE); Planck 2018 CMB lensing data; BAO data from 6dF, SDSS DR7, and SDSS DR12; Pantheon SNIa data; SDSS DR12 RSD data; DES-Y1 3x2pt data; and priors on $S_{8}$ derived from KiDS and HSC data. The SH0ES $H_{0}$ measurement is not included here. Upper and lower limits are quoted at $95 \%$ CL. With SH0ES excluded, there is no evidence for the EDE component. These constraints are computed with the credible-interval approach discussed near the beginning of Sec. [VI] and can be compared with the equal-tail limits presented in Table IX] (the mean values are, of course, identical in the two tables). 